\title{
Evidence of Absence (v2.0) Software User Guide
}

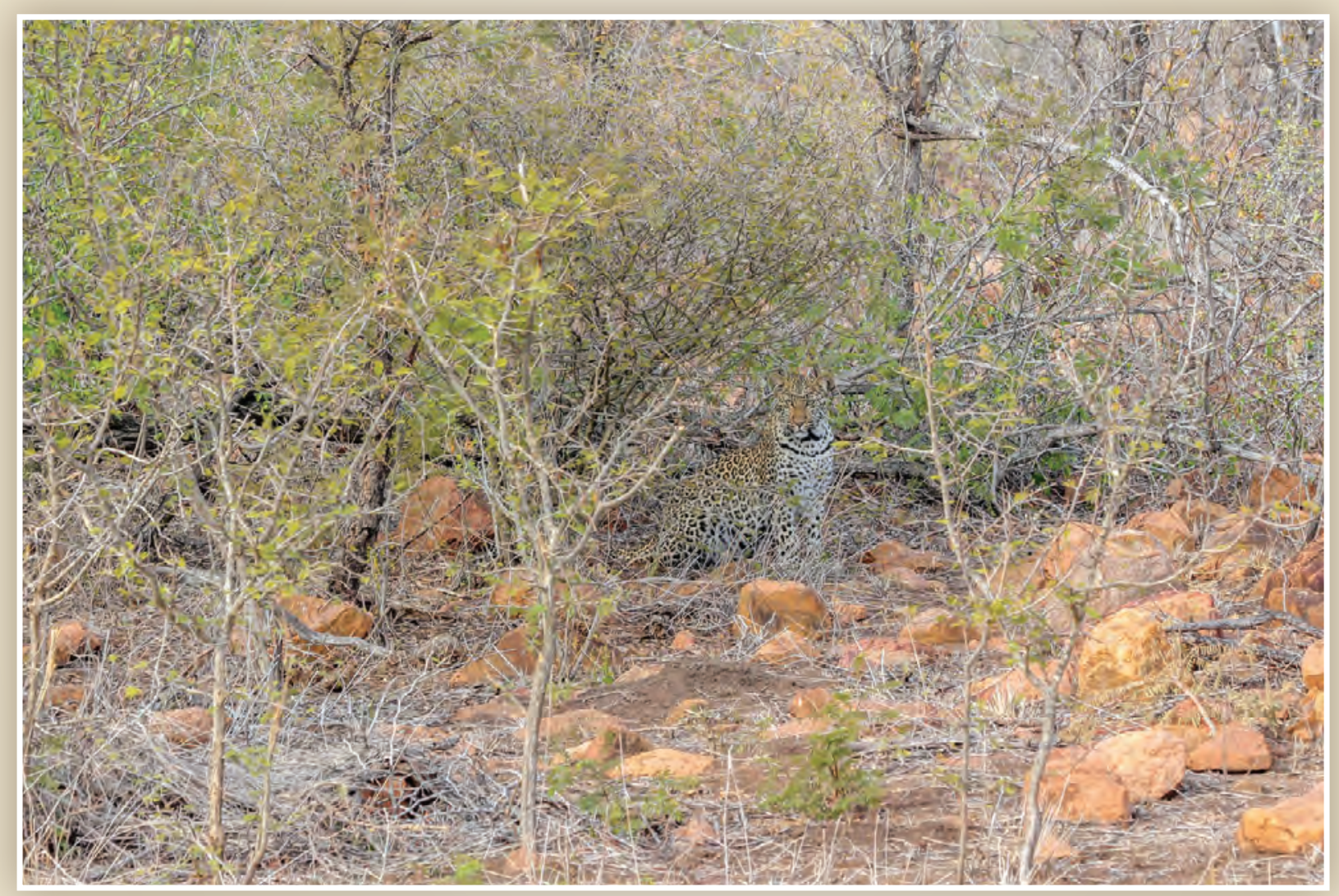

Data Series 1055

U.S. Department of the Interior

U.S. Geological Survey 
Cover: Photograph showing a leopard (Panthera pardus), Kruger National Park, South Africa, representing the difficulty in inferring absence. Photograph by Robert Peck, U.S. Geological Survey, 2016. 


\section{Evidence of Absence (v2.0) Software User Guide}

By Daniel Dalthorp, Manuela Huso, and David Dail

Prepared in cooperation with U.S. Fish and Wildlife Service

Data Series 1055

U.S. Department of the Interior

U.S. Geological Survey 


\section{U.S. Department of the Interior \\ RYAN K. ZINKE, Secretary}

U.S. Geological Survey

William H. Werkheiser, Acting Director

U.S. Geological Survey, Reston, Virginia: 2017

For more information on the USGS-the Federal source for science about the Earth, its natural and living resources, natural hazards, and the environment-visit https://www.usgs.gov/ or call 1-888-ASK-USGS (1-888-275-8747).

For an overview of USGS information products, including maps, imagery, and publications, visit https:/store.usgs.gov.

Disclaimer: The findings and conclusions in this article are those of the author(s) and do not necessarily represent the views of the U.S. Fish and Wildlife Service.

Any use of trade, firm, or product names is for descriptive purposes only and does not imply endorsement by the U.S. Government.

Although this information product, for the most part, is in the public domain, it also may contain copyrighted materials as noted in the text. Permission to reproduce copyrighted items must be secured from the copyright owner.

Suggested citation:

Dalthorp, Daniel, Huso, Manuela, and Dail, David, 2017, Evidence of absence (v2.0) software user guide: U.S. Geological Survey Data Series 1055, 109 p., https://doi.org/10.3133/ds1055.

ISSN 2327-638X (online) 


\section{Contents}

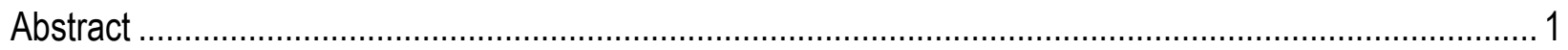

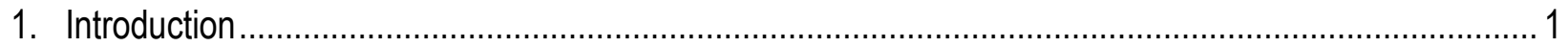

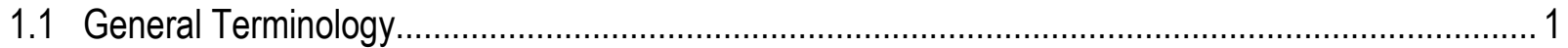

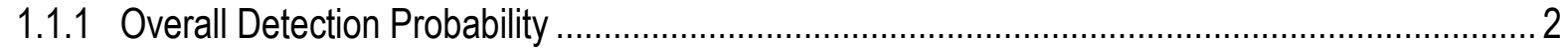

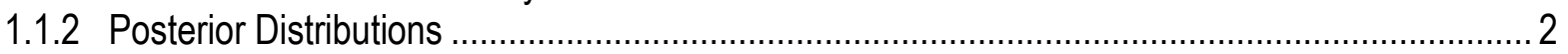

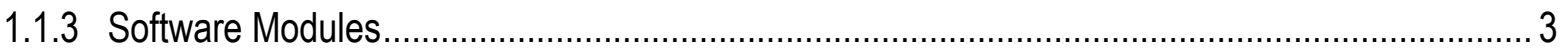

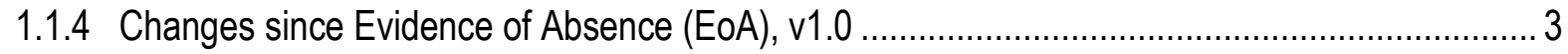

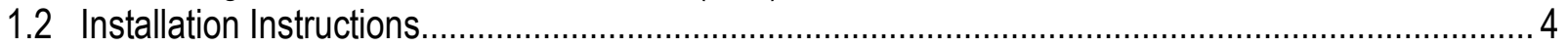

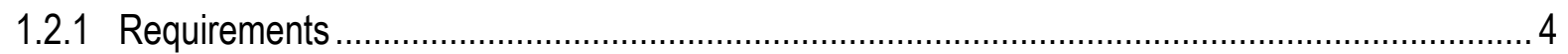

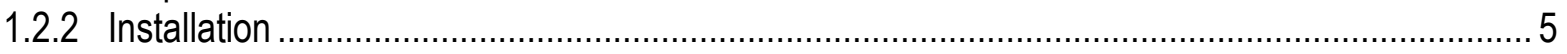

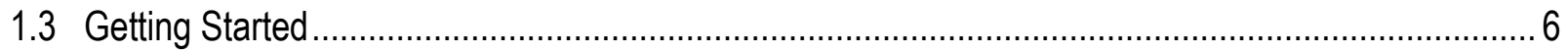

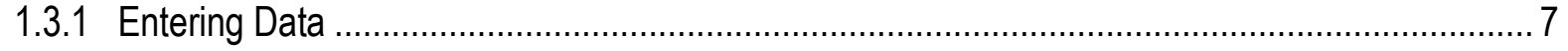

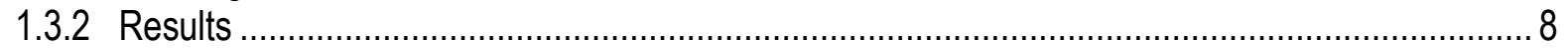

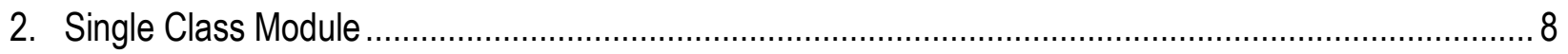

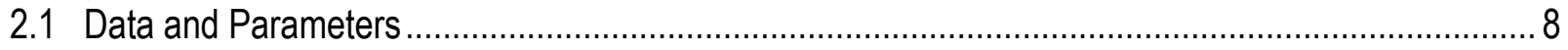

2.1.1 Search Schedule and Coverage ..................................................................................

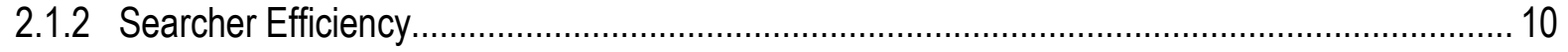

2.1.3 Persistence Distribution.............................................................................................. 14

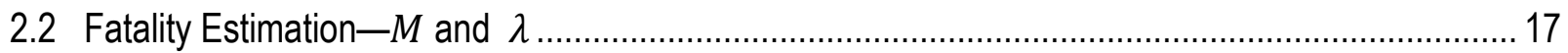

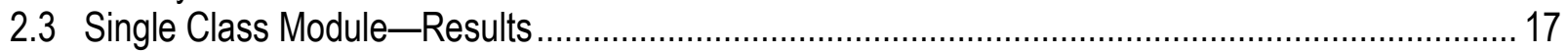

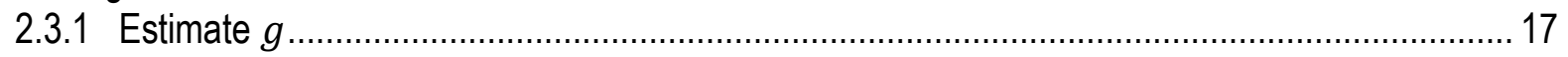

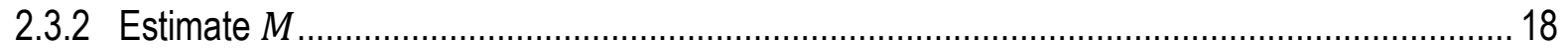

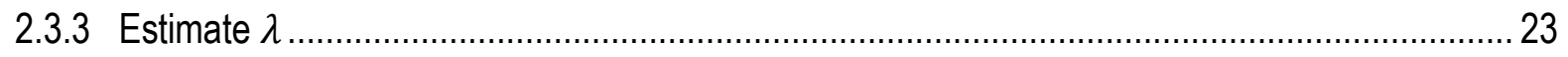

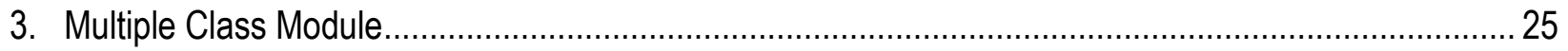

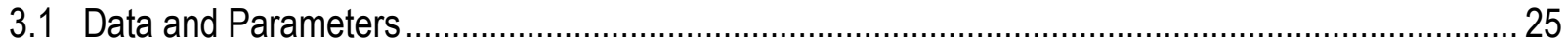

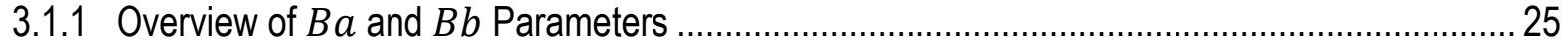

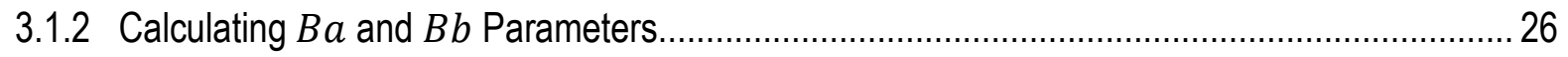

3.2 Multiple Class Module-Results......................................................................................... 27

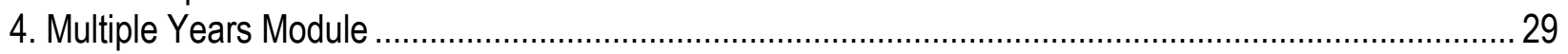

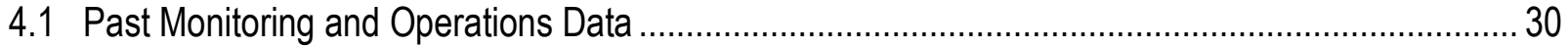

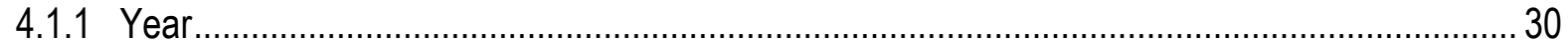

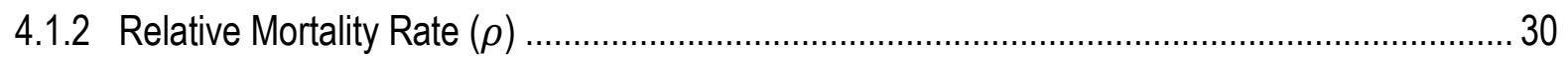

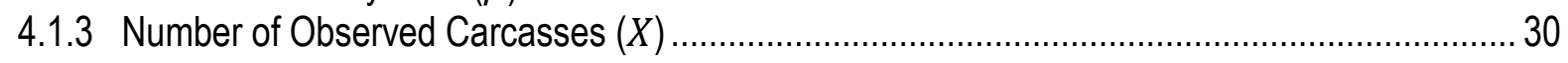

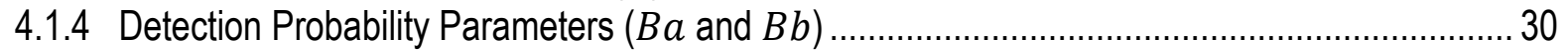

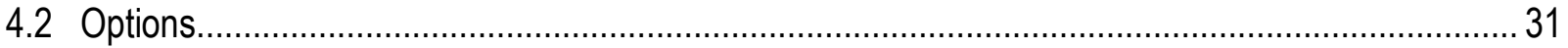

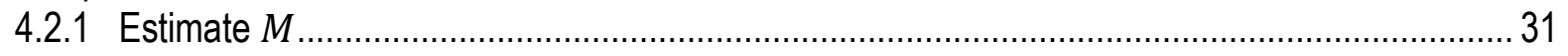

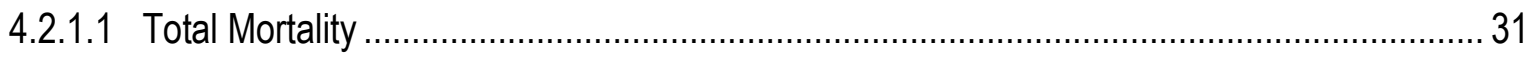

4.2.1.2 Track Past Mortality ............................................................................................ 34

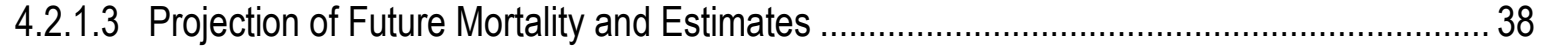

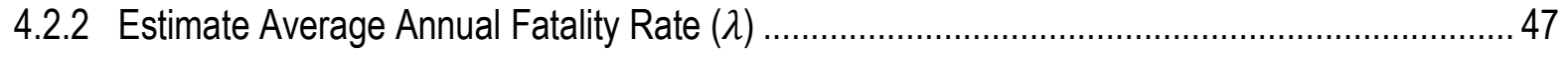




\section{Contents-Continued}

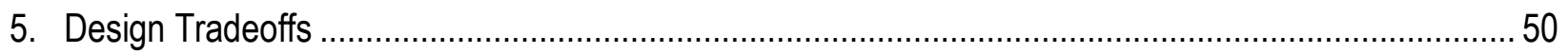

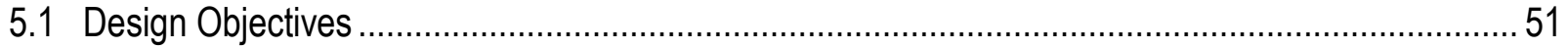

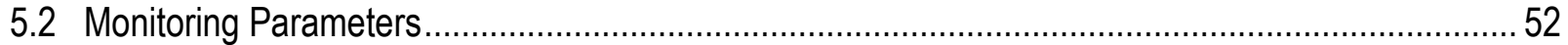

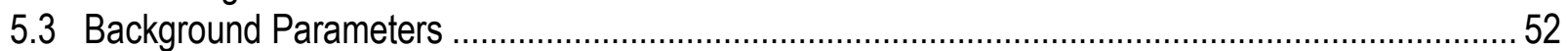

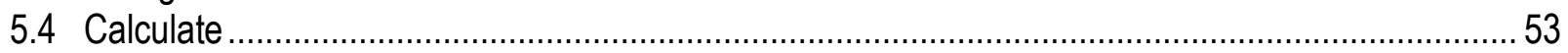

5.4.1 Design for Detection Probability .................................................................................... 53

5.4.1 Design for Credibility Level...................................................................................... 56

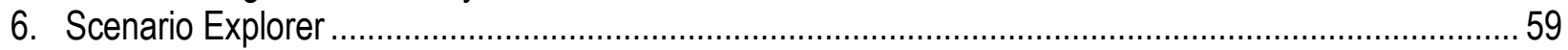

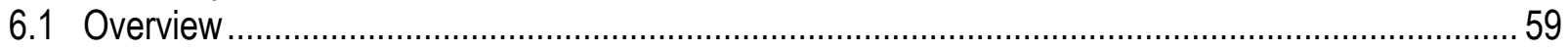

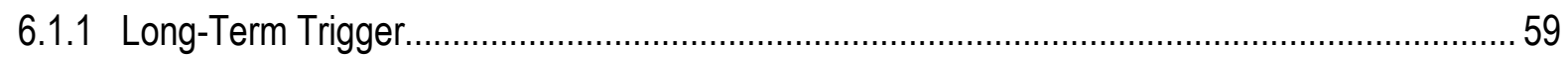

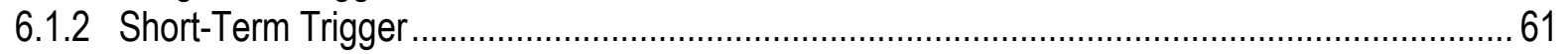

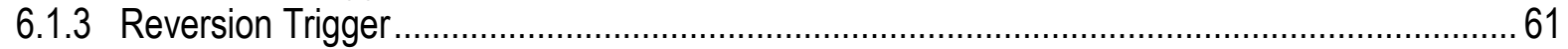

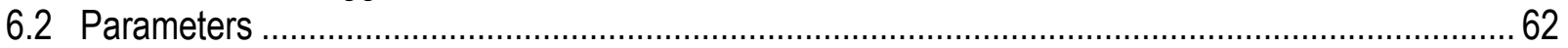

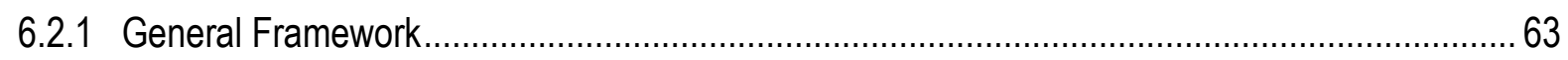

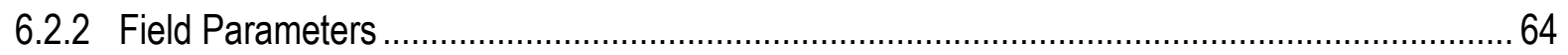

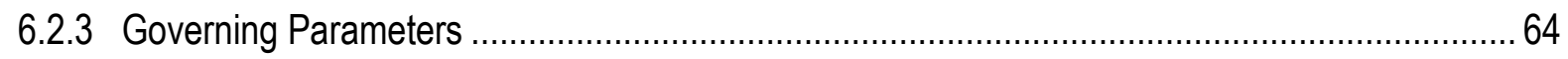

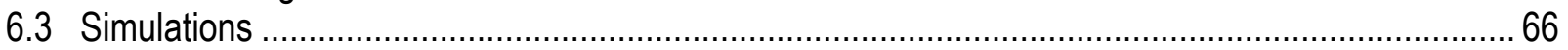

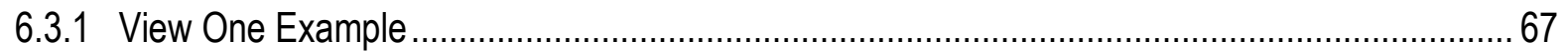

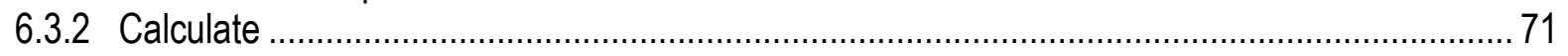

6.3.2.1 Example: Default parameters but with reversion trigger and incremental

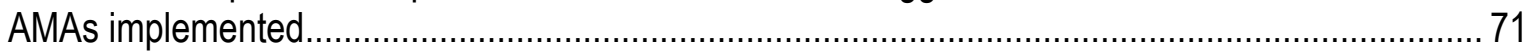

6.3.2.2 Example: Action of Long-Term Trigger and Frequency of Short-Term Triggering

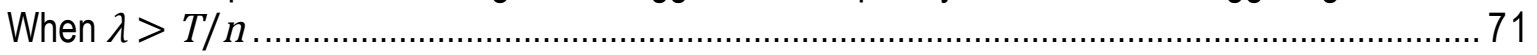

6.3.2.3 Example: Action of Long-Term Trigger and Frequency of Short-Term Triggering

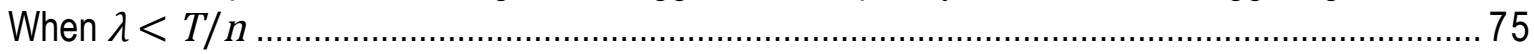

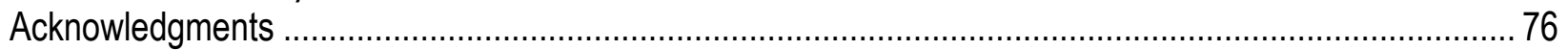

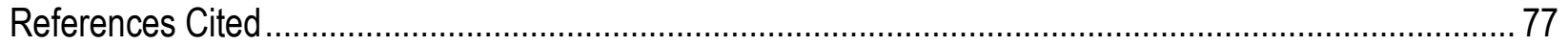

Appendix A. Estimator of Overall Detection Probability $(g)$-Single Year, Single Class ............................. 80

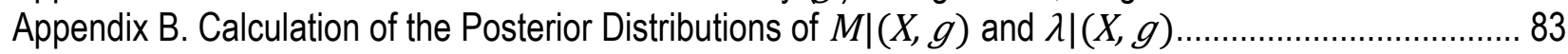

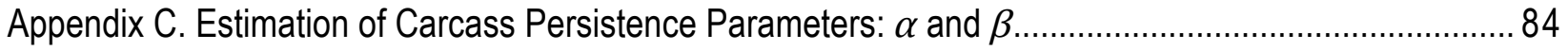

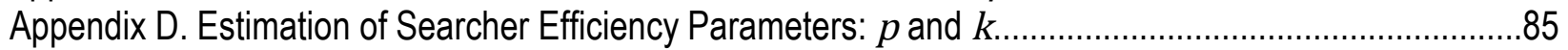

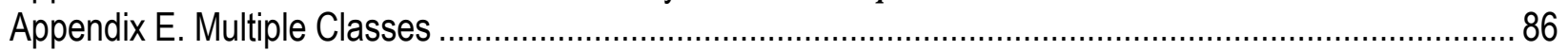

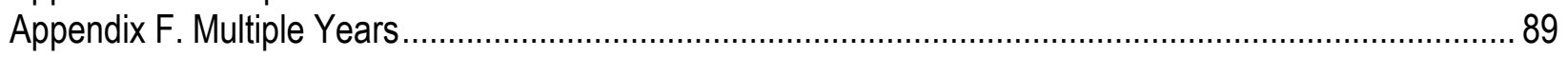

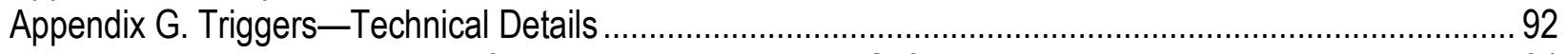

Appendix H. Parameterizations of Distributions Used in the Software .................................................... 94

Appendix I. Functions and Data Available from R Command Line ........................................................ 95

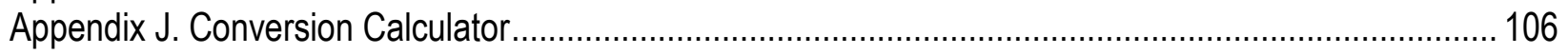

Appendix K. Comparison with Other Estimators of Detection Probability .............................................. 107 


\section{Figures}

Figure 1. Screen captures of the EoA control panel and the miniaturized R console.................................. 6

Figure 2. Screen capture of Single Class Module (input) .................................................................

Figure 3. Screen capture of form for entering data from searcher efficiency trials ..................................... 12

Figure 4. Graph generated by Evidence of Absence software showing searcher efficiency,

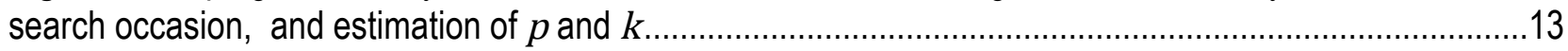

Figure 5. Screen capture of carcass persistence models .................................................................... 16

Figure 6. Graphs generated by Evidence of Absence software showing estimation of $M$ in the

Single Class Module (graphical results for $M^{*}$ ) .........................................................................19

Figure 7. Graphs generated by Evidence of Absence software showing estimation of $M$ in the

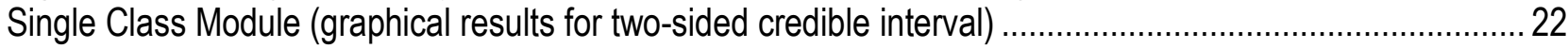

Figure 8. Graph generated by Evidence of Absence software showing posterior distribution

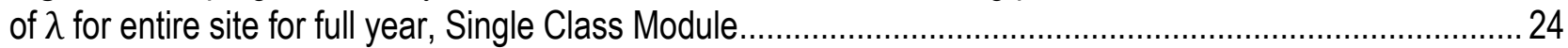

Figure 9. Screen capture of Multiple Class Module (inputs)..................................................................... 25

Figure 10. Screen capture of parameter conversions calculator ...........................................................22

Figure 11. Screen capture of Multiple Years Module (inputs). .................................................................. 29

Figure 12. Graph generated by Evidence of Absence software showing posterior distribution of $\mathrm{M}$ (Multiple Years Module)

Figure 13. Graph generated by Evidence of Absence software showing estimated cumulative mortality

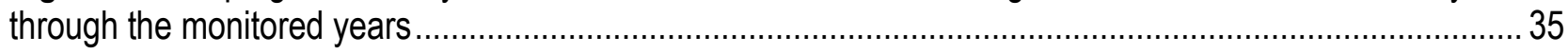

Figure 14. Box and whisker plots generated by Evidence of Absence software showing posterior distributions of $\lambda$ for each year that monitoring data were available

Figure 15. Box and whisker plots generated by Evidence of Absence software showing estimates of past mortality and projections of future mortality and mortality estimates, $\alpha=0.5$......

Figure 16. Graph generated by Evidence of Absence software showing probabilities of exceedance and triggering for each year

Figure 17. Box and whisker plots generated by Evidence of Absence software showing estimates

of past mortality and projections of future mortality and mortality estimates, 1- $\alpha=0.8$.

Figure 18. Screen capture of the Multiple Years Module, accounting for anticipated changes in operations in future years. In projection year 7 , mortality rate triples due to expansion of the site

Figure 19. Box and whisker plots generated by Evidence of Absence software showing projected mortality with anticipated tripling of fatality rate $(\rho=3)$ beginning in year 12 (or projection year 7)............. 46

Figure 20. Screen capture of Multiple Years Module, estimating average fatality rate $(\lambda)$ in past years ...... 47

Figure 21. Screen capture of Design Tradeoffs Module (inputs)............................................................ 50

Figure 22. Screen capture of $M^{*}$ vs $g$ option. Sliders control the limits of the x-axis, allowing the user to

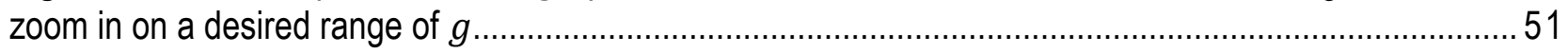

Figure 23. Graph generated by Evidence of Absence software showing user-selected persistence distribution

Figure 24. Graph generated by Evidence of Absence software showing tradeoffs between

search coverage and searcher efficiency for $g$.....

Figure 25. Graphs generated by Evidence of Absence software showing tradeoffs between

search coverage and searcher efficiency for $g$, with $I$ varying ....

Figure 26. Graph generated by Evidence of Absence software showing tradeoffs between

search coverage and searcher efficiency for 1- $\alpha$.

Figure 27. Graphs generated by Evidence of Absence software showing tradeoffs between

search coverage and searcher efficiency for $1-\alpha$, with variable $I$ values 
Figure 28. Graph generated by Evidence of Absence software showing operation of the long-term trigger ...

Figure 29. Screen capture of Scenario Explorer parameter input form with default data set

Figure 30. Screen capture of Scenario Explorer parameter options with potentially slow

simulation speeds

Figure 31. Screen capture of Scenario Explorer with default parameters but with "Reversion Trigger" and "Include incremental AMA" options selected. 66

Figure 32. Screen capture of Scenario Explorer, one example (long-term trigger)......................................68

Figure 33. Screen capture of Scenario Explorer, one example (short-term trigger). .....................................69

Figure 34. Screen capture of Scenario Explorer, one example (reversion trigger)........................................70

Figure 35. Box and whisker plots generated by Evidence of Absence software showing

Scenario Explorer results for default parameters but with active reversion trigger, incremental AMA, and 1000 simulation draws

Figure 36. Screen capture of parameters for exploring the action of short- and long-term triggers in a scenario with $\lambda=3 T / n$

Figure 37. Box and whisker plots generated by Evidence of Absence software showing Scenario

Explorer. Action of long-term trigger and frequency of short-term triggering when $\lambda=3 T / n$.

Figure 38. Box and whisker plots generated by Evidence of Absence software showing Scenario

Explorer. Action of long-term trigger and frequency of short-term triggering when $\lambda=0.5 T / n$.

\section{Tables}

Table 1. Example format of carcass persistence data.

Table 2. Results table generated by Evidence of Absence software showing estimation of $g$ in the Single Class Module.

Table 3. Results table generated by Evidence of Absence software showing estimation of $M$ in the

Single Class Module (tabular results for $M^{*}$ ).

Table 4. R command line functions for calculating key Single Class results manually....

Table 5. Results table generated by Evidence of Absence software showing Multiple Class estimates (posterior distribution of $M$ has been truncated to save space).

Table 6. Results table generated by Evidence of Absence software showing estimation of total mortality in Multiple Years Module

Table 7. Results table generated by Evidence of Absence software showing cumulative and annual mortality estimate

Table 8. Results table generated by Evidence of Absence software showing summary statistics

for future mortality projections, $1-\alpha=0.5$ (part $A$ ).

Table 9. Results table generated by Evidence of Absence software showing summary statistics for past mortality estimates, $1-\alpha=0.5$ (part $B$ )

Table 10. Results generated by Evidence of Absence software showing short-term trigger test results........ 48

Table 11. Reversion trigger test results. 


\section{Abbreviations}

AICc Akaike information criterion corrected for finite sample size

$\triangle \mathrm{AIC}$ difference between AICc value for a given model and the lowest AICc value among models tested

AMA adaptive management action taken in response to a monitoring trigger

.csv comma-separated values (format for some input files)

$\mathrm{CDF} \quad$ cumulative distribution function

CCDF complement of the cumulative distribution function or $1-\mathrm{CDF}$

CI confidence interval or credible interval (depending on context)

CP carcass persistence

CPmax the earliest time a persistence trial carcass is noted to be missing

CPmin the latest time a persistence trial carcass is known to be present

CRAN comprehensive R archive network (for downloading R statistical software and supplemental packages)

EoA evidence of absence software

IQR interquartile range; bounds of the central $50 \%$ of a distribution; the 0.25 and 0.75 quantiles of a distribution

ITP incidental take permit

JAGS just another Gibbs sampler (for calculating Bayesian statistics)

LRT likelihood ratio test

PDF probability density function

PI prediction interval

.rds $\quad$ serialized $\mathrm{R}$ data object (format for some input files)

SE searcher efficiency 


\section{Variables and Parameters}

$\alpha \quad$ significance level (distinguished from shape parameter by context)

$1-\alpha \quad$ credibility level, confidence level (depending on context)

$\alpha, \beta \quad$ shape and scale parameters for persistence distributions

a spatial coverage, fraction of carcasses falling into searched area

$B a, B b \quad$ beta distribution parameters that characterize the estimated detection probability, so $\hat{g} \sim \operatorname{beta}(B a, B b)$

$d w p \quad$ density weighted proportion; fraction of the total carcasses that arrive in a searched area

$g \quad$ overall detection probability

I search interval; number of days between searches

$I_{r} \quad$ assumed search interval for calculation of $r$

$k \quad$ factor by which searcher efficiency decreases with each successive search

$\lambda \quad$ mortality rate

$M \quad$ mortality or number of fatalities

$M^{*} \quad$ estimated mortality or number of fatalities

$p \quad$ searcher efficiency, probability of observing a carcass that is present in the searched area at the time of the search

$\rho \quad$ relative mortality rate

$\rho_{0} \quad$ the assumed relative mortality rate under minimization measures in effect at the beginning of a permit compared to the assumed mortality rate when minimization measures are not effect

$\rho_{\infty} \quad$ the assumed relative mortality rate under adaptive management measures designed to avoid future fatalities

$r \quad$ probability that a carcass persists unscavenged until the first search after arrival

span the length of the monitoring period

$\mathrm{T}$ total cumulative take limit for a long-term incidental take permit

$\tau \quad$ annual take limit

$v \quad$ temporal coverage, fraction of total carcasses that arrive during the monitored period

$X \quad$ number of carcasses observed in searches 


\title{
Evidence of Absence (v2.0) Software User Guide
}

\author{
by Dan Dalthorp ${ }^{1}$, Manuela Huso1, and David Dail2
}

\begin{abstract}
Evidence of Absence software (EoA) is a user-friendly software application for estimating bird and bat fatalities at wind farms and for designing search protocols. The software is particularly useful in addressing whether the number of fatalities is below a given threshold and what search parameters are needed to give assurance that thresholds were not exceeded. The software also includes tools (1) for estimating carcass persistence distributions and searcher efficiency parameters ( $p$ and $k$ ) from field trials, (2) for projecting future mortality based on past monitoring data, and (3) for exploring the potential consequences of various choices in the design of long-term incidental take permits for protected species. The software was designed specifically for cases where tolerance for mortality is low and carcass counts are small or even 0 , but the tools also may be used for mortality estimates when carcass counts are large.
\end{abstract}

\section{Introduction}

\subsection{General Terminology}

The software addresses the general problem of estimating numbers of fatalities over an extended period of time using systematic counts of carcasses and adjustments of the carcass counts to account for imperfect detection. Imperfect detection may be due to any of several possible detection biases, for example: (1) search teams fail to find carcasses that are present in the searched area at the time of the search, (2) scavengers remove carcasses before searches are conducted, (3) carcasses fall outside the searched area, or (4) fatalities occur outside the monitored period. The detection rate $(g)$ is estimated primarily from results of field trials in which carcasses are placed at known locations within the searched areas at the site and monitored for persistence times and for evaluating the efficiency of search teams in detecting carcasses that are not scavenged. Combining the number of carcasses $(X)$ found in the systematic carcass searches with information about the detection rate, Evidence of Absence software (EoA) estimates the total mortality $(M)$ and quantifies the uncertainty associated with the estimation.

\footnotetext{
${ }^{1}$ U.S. Geological Survey.

${ }^{2}$ Oregon State University.
} 


\subsubsection{Overall Detection Probability}

The EoA software estimates the overall probability $(g)$ of detecting a carcass that arrives at a site (or group of sites) during the monitoring season(s) or longer period of inference. If $g$ is known exactly with no uncertainty, a good estimator for the total mortality would simply be:

$$
\widehat{M}=X / g
$$

where $X$ is the number of carcasses observed, and $g$ is the overall detection probability.

In practice, $g$ is never known exactly and must be estimated. To calculate an estimate $(\hat{g})$ of the overall detection probability, EoA uses a generalized estimator, which takes into account scavenging rates, searcher efficiency, search schedule, and spatial and temporal search coverage. The model can accommodate a number of realistic scenarios that many commonly used estimators cannot, for example, a scavenging rate that varies with carcass age, a decrease in searcher efficiency for carcasses that have been missed in previous searches, and an irregular search schedule. Due to its generality, the model for estimating $g$ is fairly complicated. Details are presented in appendix A.

\subsubsection{Posterior Distributions}

Substituting the estimated detection probability, $\hat{g}$, for the actual (unknown) $g$ in equation (1) gives a rough point estimate of mortality $(\widehat{M}=X / \hat{g})$ that can be used as a "reality check" for the estimates provided by EoA (or other model) but should not be considered a rigorous or particularly informative estimate. The estimator can be strongly biased when $g$ is small, does not give accurate confidence intervals when $X$ is small, and collapses entirely for $X=0$ (Huso and others, 2015). As an alternative, after accounting for uncertainty in both $g$ and $\widehat{M} \mid(X, g)$ (that is, the estimated mortality $(\widehat{M})$ given the carcass count $(X)$ and detection probability $(g)$ ), EoA calculates a posterior distribution (or simply posterior), which is a quantitative representation of what is known about the total number of fatalities $M$ (technical details are provided in appendix B). The posterior distribution gives the probability that the number of fatalities equals any given non-negative integer, $m$. Point and interval estimates of $M$ can be extracted from the posterior in myriad ways. The most commonly used statistics are a point estimate, $M^{*}$, and credible intervals, which are the Bayesian analog to confidence intervals in classical statistics.

Mortality is estimated as $M^{*}$, which is defined as the minimum number of fatalities such that $P\left(M \leq M^{*} \mid X=x ; \hat{g}\right) \leq 1-\alpha$ for the user's choice of credibility level, $1-\alpha . M^{*}$ can be interpreted as a $100(1-\alpha) \%$ credible upper bound on the number of fatalities or as a statement like: "Based on the search data, we can assert with $100(1-\alpha) \%$ credibility that there were no more than $M^{*}$ fatalities during our period of interest." The value of $1-\alpha$ governs how conservative $M^{*}$ is as an estimate of $M$. Larger values of $1-\alpha$ yield larger $M^{*}$ and stronger assurance that the actual number of fatalities is less than $M^{*}$. With a value of $1-\alpha=0.5, M^{*}$ is the median of the posterior and can be taken as a point estimate of $M$ with roughly equal probabilities that $M \geq M^{*}$ or $M<M^{*}$.

A $100(1-\alpha) \%$ credible interval $(\mathrm{CI})$ is constructed from the posterior with interpretation that the probability that $M$ is in the interval is (approximately) $1-\alpha$. The algorithm that EoA uses for constructing a $\mathrm{CI}=\left[M_{l}, M_{u}\right]$ for $M$ is to define the lower bound, $M_{l}$, as the smallest integer such that $P\left(M \leq M_{l}\right)>\alpha / 2$ and the upper bound as the smallest integer, $M_{u}$, such that $P\left(M \in\left[M_{l}, M_{u}\right]\right) \geq$ $1-\alpha$. 


\subsubsection{Software Modules}

The Evidence of Absence (v2.0) software includes five modules:

1. Single Class - for estimating detection probability and mortality for a single year (or monitored period) and single search class with submodules for analyzing carcass persistence and searcher efficiency trials;

2. Multiple Classes - for combining data from several search classes within a site or from several distinct sites into an estimate of total mortality;

3. Multiple Years - three components for:

a. estimating cumulative take over several years,

b. projecting future take (based on past monitoring data and future monitoring and operations plans), and

c. hypothesis tests and CIs for the annual mortality rate $(\lambda)$ over one or a few years;

4. Design Tradeoffs - for designing a monitoring protocol to optimize search resources, including search coverage, searcher efficiency, and search interval; and

5. Scenario Explorer-for predicting future mortality and compliance with incidental take permits (ITPs).

\subsubsection{Changes since Evidence of Absence (EoA), v1.0}

EoA v2.0 is a substantial upgrade from v1.0 of the software (Dalthorp and others, 2014). Major changes include:

1. EoA, v2.0 is bundled as an R package and does not depend on an interface between $\mathrm{R}$ and Microsoft Excel ${ }^{\circledR}$ mediated by statconnDCOM and RExcel. The newer version is substantially easier to install and is compatible with Microsoft Windows ${ }^{\circledR}$, Mac OS, and Unix-like operating systems and with both 32-bit and 64-bit R (section 1.2).

2. The default prior distribution used for calculating the posterior distribution for $M$ has been changed from uniform to an integrated reference prior, which is often referred to as the objective prior (Berger and others, 2012). In most cases, the two priors give the same posteriors. When they differ, the objective prior is more accurate in the sense that when $M^{*}$ is calculated using the objective prior $P\left(M>M^{*}\right)$ is closer to $\alpha$ than it is when the uniform prior is used (appendix B). The difference is most noticeable when $X$ is small, when the objective prior frequently gives a smaller $M^{*}$ than does the uniform prior. In addition, in EoA v2.0, users are no longer offered the option of entering custom or informed prior except via the command line in $\mathrm{R}$.

3. A new submodule for analyzing carcass persistence field trial data simplifies the process for fitting persistence distributions and entering the parameter estimates into EoA (section 2.1.3). In addition, a new algorithm for simulating persistence times (appendix $\mathrm{C}$ ) gives more accurate and reliable results than with EoA v1.0. 
4. A new submodule for analyzing searcher efficiency data includes an option for simultaneous estimation of $k$ and $p$ parameters from field trial data (section 2.1.2), and new algorithm for simulating searcher efficiencies (appendix D) gives more accurate and reliable results than EoA v1.0 gives.

5. The multiple class module features streamlined data entry that is more convenient and flexible than with EoA v1.0, and the estimator for $M$ is more accurate when $X$ is small (appendix E).

6. The multiple years module includes new tools for estimating the mean fatality rate $\lambda$ by year (section 4.2.2); testing long-term, short-term, and reversion triggers (Dalthorp and Huso, 2015); and projecting of future mortality (section 4.2.1.3). Technical details are provided in appendix F.

7. The new design tradeoffs module includes an option for designing monitoring protocol to attain a target $g$ and a new tool for viewing $M^{*}$ as a function of $g, X$, and $\alpha$ (section 5).

8. The long-term scenario explorer is an entirely new module with tools for investigating the consequences of decisions about regulatory framework parameters in the context of long-term ITPs (section 6). Technical details are provided in appendix G.

\subsection{Installation Instructions}

\subsubsection{Requirements}

- R software, version 3.3.0 or higher

- JAGS, version 4.2.0 (with Rtools33) or higher

- R packages:

actuar (Dutang and others, 2008)

gs l (Hankin, 2006)

matrixstats (Bengtsson, 2016)

R6 (Chang, 2016)

rjags (Plummer, 2016)

tcltk2 (Grosjean, 2016)

tensorA (Boogart, 2010)

tkrplot (Tierney, 2011)

VGAM (Yee, 2017) 


\subsubsection{Installation}

There are several steps to installing the software. Do not skip any steps.

\section{R Software, Version 3.3.0 or Higher}

The new EoA requires version 3.3.0 (or higher) of the $\mathrm{R}$ statistical computing software (R Core Team, 2016), which is free and open source. To install, use your Internet browser to navigate to https: / / www.r-project.org/ and click following links: "CRAN mirror" (choose the nearest site) and "Download R for ...." If you are installing on a computer with Microsoft Windows ${ }^{\circledR}$, click "base", and "Download R x.x.x for Windows". You may need to be logged in with administrative privileges to install. If installing on a computer with $\mathrm{Mac}^{\circledR}$ or a UNIX ${ }^{\mathbb{R}}$-like operation system, follow the instructions provided at the website.

If you are updating your copy of $\mathrm{R}$ from a previously installed version, the new version will be installed alongside the old version rather than on top of it, and both versions will be operational. Unless you know the old version will be needed for some specific purpose in future, uninstalling it is recommended. This can be done using the Control Panel in Windows ${ }^{\circledR}$. Add-in R packages that the user downloaded and installed in an earlier version will not be available in the updated version unless they are either reinstalled or copied from the library file of the previous installation to the library file of the new installation and updated in the new version of R using update.packages (checkBuilt $=$ TRUE, ask = FALSE).

JAGS (Plummer, 2003), Version 4.2.0 (with Rtools33) or Higher

JAGS is free and open source software that is independent from $\mathrm{R}$ but will run in the background when needed by $\mathrm{R}$ or EoA. Use your Internet browser to navigate to https://sourceforge.net/projects/mcmc-jags/files/JAGS/4.x/Windows/ to download and install the most recent version of JAGS with Rtools33. Installation may require administrative privileges on computer.

\section{Third-Party R Packages}

Several third-party $\mathrm{R}$ packages are required and are installed automatically when EoA is first used (section 1.3). Alternatively, the packages can be installed from within $\mathrm{R}$ by copy-and-paste of the following commands into the R command line:

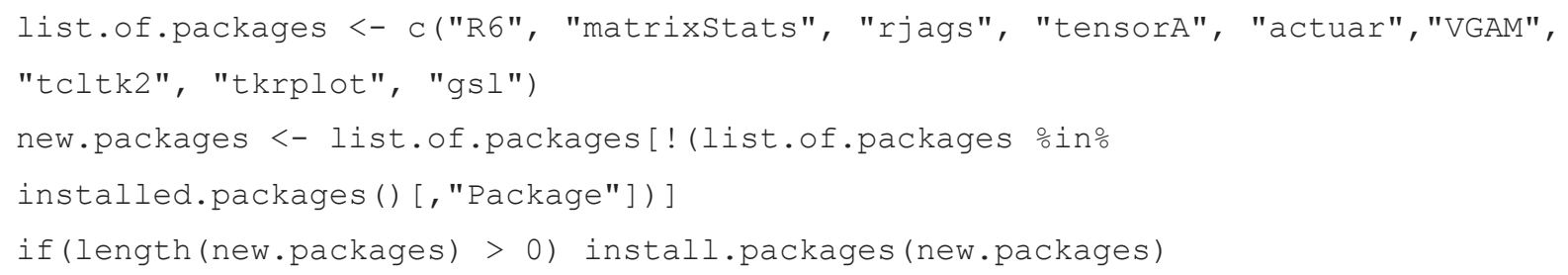

Depending on the current status of your $\mathrm{R}$ installation, it could take anywhere from a fraction of a second to several minutes to install and/or check the required packages. If new packages need to be installed, a dialog box may ask you to select a CRAN mirror. Choose the nearest location. Next, you might be asked if you want to use a personal library instead (or something to that effect). Choose "Yes." Once these packages have been installed, you will not need to go through the wait again. 


\section{EoA Package}

The EoA software is bundled as an R package. To complete the installation for Windows ${ }^{\circledR}$, download the "eoa X.x.x.x.zip" file to a convenient location but do not unzip. Select "Install package(s) from local zip files..." from the "Packages" menu in R. Navigate to the eoa zip folder and double click or select and click “Open." If installing to $\mathrm{Mac}^{\circledR}$ or UNIX ${ }^{\circledR}$-like operating system, download the source package eoa_2.0.tar.gz and run install.packages (path_to_file, repos = NULL), where path_to_file is the path to the EoA folder. R may prompt Mac ${ }^{\bar{B}}$ users to install "Command Line Tools" for Xcode, which are necessary for installing packages from source. Mac ${ }^{\circledR}$ users may al so need to install xquartz from https://www.xquartz.org/ to enable communication between the Mac ${ }^{\circledR}$ OS and the $\mathrm{R}$ graphics windows.

\subsection{Getting Started} line:

To run EoA once it has been installed, enter the following two commands onto the R command

\section{library (eoa)}

eoa ( )

The first time EoA is run, it checks whether all the required $\mathrm{R}$ packages have been previously installed. If some are missing, you might be asked to select a CRAN mirror. Choose the nearest location. Next, you might be asked if you want to use a personal library instead (or something to that effect). Choose "Yes." Once these packages have been installed, you will not need to go through the wait again.

Upon starting EoA, the R console will shrink to a small box and the EoA control panel will appear (fig. 1). The R console may be safely ignored, or it may be expanded for doing custom calculations or checking for error messages. However, closing the R console window will end the EoA session.
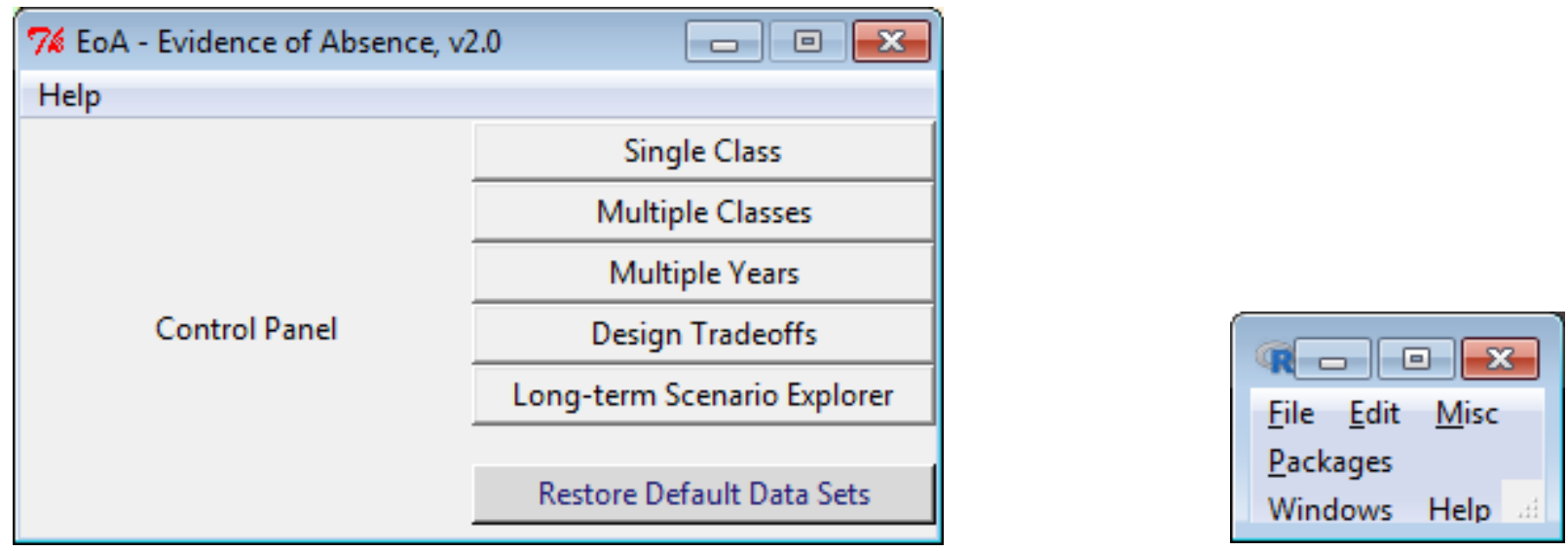

Figure 1. Screen captures of the EoA control panel and the miniaturized R console. 
When a module is opened by clicking on one of the buttons in the control panel, the control panel will close and a new window will open with a set of parameter values automatically entered. The first time a module is opened, a set of default parameters values is loaded. These values represent data from a fictitious but plausible site. The values have no particular significance and should not be interpreted as recommendations or as meaningful in any way. They serve as a pre-loaded, workable example for learning the software and as a convenient starting point for discussions about interpretation of input, results, or software performance. Parameter sets may then be edited. Upon performing calculations, the active parameter set is saved for reloading the next time the module is opened. If desired, the default parameter set for an open module may be reloaded by selecting Restore defaults from the Edit menu. The default parameter sets for all modules may be reloaded by selecting Restore Default Data Sets from the Control Panel menu

When a module is closed by clicking the "close" icon (red " $\mathrm{X}$ " in the upper right corner), clicking the Close button or entering $<$ Alt + F4 $>$, the EoA control panel will reopen. The next time the module is opened, the parameter set that was active the last time calculations were performed will be loaded.

\subsubsection{Entering Data}

\section{Text Boxes}

Live error-checking is performed on data entered into textboxes in the modules. Errors are flagged by coloring the background of the textbox - yellow for invalid entries (for example, negative number of carcasses discovered) and blue for values that are incompatible with other data (for example, lower bound that exceeds upper bound).

Tables

In most cases, users can type values directly into cells in data entry tables and use the arrow keys for navigating among cells. In addition, pressing $<\mathrm{Ctrl}+\mathrm{a}>$ adds a new row to a table and $<\mathrm{Ctrl}+\mathrm{d}>$ deletes a row. Data can also be entered into most tables using $<$ paste $>$. The upper left corner of the paste region will be the active cell (where the cursor is), and pasted data will overwrite data in the paste region. In some tables, new data can only be entered using a text or .csv file.

Complete input parameter sets can be saved by selecting Save to file from the Edit menu for a module and later uploaded by selecting Read from file from the Edit menu. 


\subsubsection{Results}

Graphs

EoA has a rich array of functions for creating graphical displays of results in $\mathbf{R}$ Graphics devices. The $\mathrm{R}$ graphing windows remain open until they are closed by the user or $\mathrm{R}$ is closed. If a new graph is created when a graphing window is already open, the first graph is typically erased and overwritten by the new graph. Graphs can be saved in a number of different ways:

i. on the File menu, click Save as..., then select a format and type a file name,

ii. on the File menu, click Copy to clipboard as a bitmap or as a metafile (Metafiles are efficient, clear, scalable, and can be pasted seamlessly into Microsoft Word ${ }^{\circledR}$ or Powerpoint $^{\circledR}$ but may not be as easily compatible with other programs), or

iii. right-click the graph and copy to clipboard or save to a file.

Text

Summary statistics and tables of results are also written to $\mathrm{R}$ data windows that can be saved as text files by clicking Save to File on the File menu in a results window or can be copied to the clipboard for pasting into other programs.

\section{Single Class Module}

\subsection{Data and Parameters}

Central to mortality estimation are a number of parameters that determine the overall probability of detecting a carcass (fig. 2). The parameters fall into three categories - search schedule and coverage, searcher efficiency, and persistence distribution. 


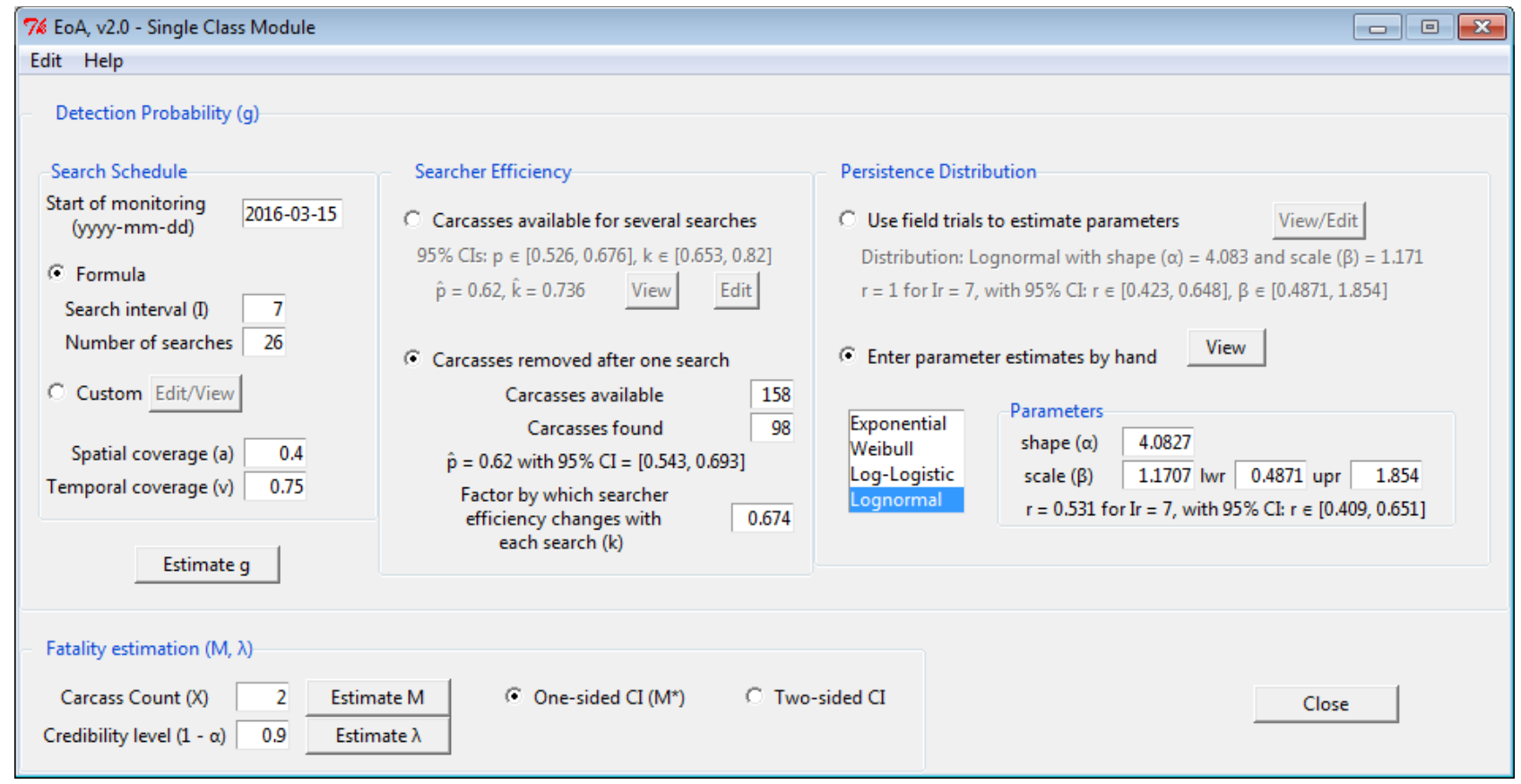

Figure 2. Screen capture of Single Class Module (input).

\subsubsection{Search Schedule and Coverage}

Start of monitoring is the date that marks the beginning of the monitored period. The model assumes that all carcasses that are discovered during the monitoring season arrived after the start of monitoring. In practice, this typically means that either the monitoring begins before carcass arrivals would be expected to begin, or the monitoring season begins at time $t=0$ with a "cleanout search" in which carcasses arriving before the start of monitoring are removed from the search plots and not included in the carcass count for the monitored period because they did not arrive during the implied period of inference. If the intended period of inference extends beyond the monitored period (for example, the user is interested in total annual mortality but monitors only from April 1 through October 31 ), the user's assumption about what fraction of carcasses arrive within the monitored period can be entered as the $v$ parameter, which is described later in this section. Formula option for specifying the search schedule is used if searches are conducted at uniform intervals throughout the monitored period, in which case the Search interval (I) is specified along with Number of searches (not including the first search at the start of the monitored period, $t_{0}$ ). Note that the search interval is the number of days between visits to a given turbine. For example, if search teams visit two sets of turbines on alternate days, searches are conducted every day but the search interval would be entered as 2 days because each turbine is visited every other day.

Custom option for specifying the search schedule is used if search intervals were irregular. Search dates are read from a text or .csv file ("comma separated values") using the Read command from the Edit menu or pasted from the clipboard using the Paste command from the Edit menu. Dates that are read from a file must be entered in a single column with a column name in the first row and 0 as the first date. 
Spatial coverage (a) is the fraction of the total carcasses expected to arrive in the searched area. For example, if there are 10 turbines and 3 are intensively searched to a wide enough radius to encompass all carcasses and there is no unsearched area within that search radius, then $a=0.3$. Another example - if there is one turbine that is only partially searched, $a$ is the fraction of carcasses that are expected to land in the searched area around the turbine. $a$ must be in the interval $(0,1]$. It must be emphasized that $a$ is not the fraction of the area that is searched but the fraction of carcasses arriving in the searched area. The number of carcasses arriving at a given distance from a turbine tends to decrease with distance while the area increases so that an area nearer to a turbine generally accounts for more carcasses than does an area of equal size at a greater distance from a turbine. Thus, $a$ should be a density-weighted proportion (dwp) of the area sampled (Huso and Dalthorp, 2014). When the number of carcasses of the target species is too small to model the relationship between distance and carcass density, a surrogate species is often used to estimate $a$. If no surrogate is available, then a mechanistic model or a model fit at a similar site is sometimes used. The $a$ parameter can have a strong influence on mortality estimates, and its assumed value should be chosen with care. This is true regardless of which estimator is used, whether it be EoA or any other estimator.

Temporal coverage $(v)$ is the fraction of the total carcasses expected to arrive during the monitored period. The parameter is used to extrapolate beyond the monitored period if desired. For example, if the carcass arrival rate is constant throughout a full year but the monitoring season is only 3 months, the temporal coverage for the year would be $3 / 12=0.25$. In some situations, though, carcass arrival may be zero during certain seasons. For example, there may be no mortality before April 1 or after October 31. In that case, if the monitoring season extended from April 1 to October 31, the temporal coverage for the year would be $v=1$ because no carcasses arrive outside the monitored season. The $v$ parameter can have a strong influence on mortality estimates extrapolated beyond the monitored period, and its assumed value should be chosen with care. If interest is solely in the number of fatalities occurring during the monitored period, use $v=1$.

\subsubsection{Searcher Efficiency}

Searcher efficiency is the probability of observing a carcass that is present in the searched area at the time of the search. Searcher efficiency values are typically determined by searcher efficiency (SE) trials conducted on an ongoing basis during routine searches throughout the season (Huso and others, 2012). Searcher efficiency may depend on carcass age as older carcasses are harder to find as they accumulate dust or debris, fall deeper into vegetation, get blown against objects or into holes, decay, or get partially scavenged. In addition, carcasses missed in one search tend to be more likely to be missed in subsequent searches because the easy-to-find carcasses are preferentially removed in the first searches after carcass arrival, leaving mostly the harder-to-find carcasses available in subsequences searches. EoA accounts for a non-constant searcher efficiency using two parameters, $p$ and $k$. 
The searcher efficiency for carcasses on the first search after arrival is defined as $p$. The decrease in searcher efficiency for aging carcasses is modeled using the $k$ parameter, which is defined as the decrease in searcher efficiency with each successive search. For example, the probability of detecting a carcass that is present in the first search after arrival is $p$. If the carcass is not found in the first search but is still present at the time of the second search, the probability of detection decreases from $p$ to $k p$. If it is missed again on the second search but persists until the third search, the probability of detection decreases by another factor of $k$ to $k^{2} p$ and similarly decreases by a factor of $k$ with each search. If searcher efficiency is the same no matter how long a carcass has been in the field or how many times it has been missed, then $k=1$. If a carcass missed in the first search after arrival has no chance of being found in later searches (or is not included in the carcass counts), then $k=0$.

To estimate $p$, EoA requires the user to enter searcher efficiency trial data, including the number of carcasses available for discovery on the first search after carcass placement and the number of carcasses actually discovered. The user has the option of entering additional data for estimation of the $k$ parameter (option: Carcasses available for several searches) or may enter an assumed value of $k$ manually (option: Carcasses removed after one search).

\section{Option: Carcasses Available for Several Searches}

To estimate $k$, searcher efficiency trial carcasses that are not discovered on the first search after being placed should be left on the ground for discovery during later searches. The number of carcasses available for discovery and the number found on each search occasion in the field trial can be entered into the software by selecting the Carcasses available for several searches option in the Searcher efficiency section of the Single Class Module. Click the Edit box to open an editable table and enter the search trial data (fig. 3). Navigate the table using the arrow keys, the $<$ Enter $>$ key, or the mouse. Pressing $<\mathrm{Ctrl}+\mathrm{d}>$ deletes the active row, and $<\mathrm{Ctrl}+\mathrm{a}>$ inserts a blank row below the cursor. When the data have been entered, the user may click the View button to see a graphical display of the data and the fitted model (fig. 4). The graph shows the fraction of carcasses discovered on each search occasion, with the number of carcasses available proportional to the size of the graphing symbol. Red dotted lines mark 95\% CIs for the searcher efficiency on each successive search after carcass arrival. Clicking OK fits the searcher efficiency model, loads the results into the parameter list, closes the searcher efficiency window, and returns control to the Single Class Module. Note that in the default data, the Carcasses Available on the second search is only 55 rather than the 60 that remained after the first search. This is likely due to scavenger removal of some of the remaining carcasses before a second search was completed. Also note in the example, there were adequate carcasses to track the searcher efficiency through 12 search occasions. This is desirable but not necessary as even 2 or 3 additional search occasions will generally provide useful estimates of $k$. 


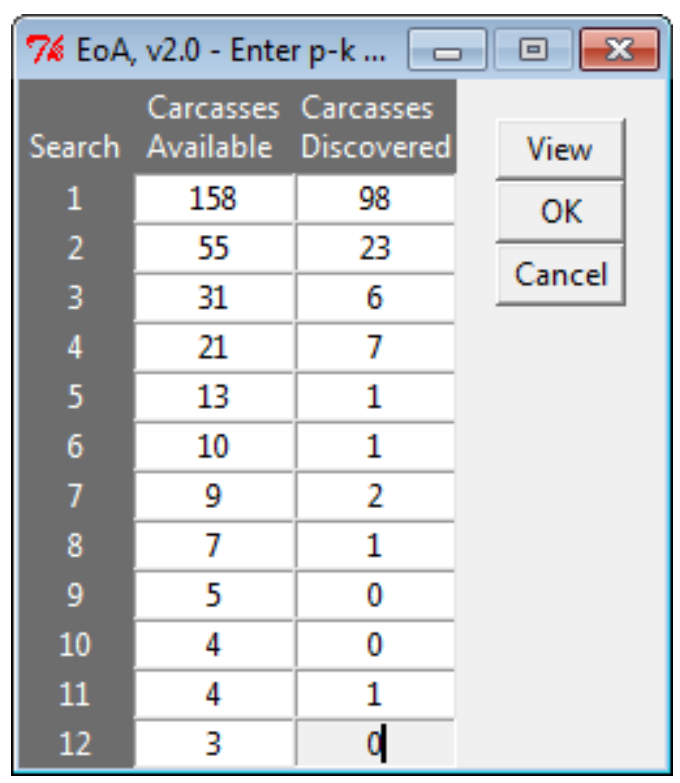

Figure 3. Screen capture of form for entering data from searcher efficiency trials. Data may be entered by using the number keys or by pasting from a spreadsheet or tab delimited text file. Rows may be added using $<\mathrm{Ctrl}+\mathrm{a}>$ or deleted using $<$ Ctrl $+d>$.

\section{Option: Carcasses Removed after One Search}

If carcasses in searcher efficiency trials are tracked for only one search so that $k$ cannot be estimated from the trial data, EoA still requires the user to explicitly enter an assumed value of $k \in$ $[0,1]$. An assumed value of $k$ that is too large — as with the Shoenfeld (2004) estimator, which implicitly assumes $k=1$-will overestimate overall detection probability $(g)$ and underestimate total mortality. An assumed value of $k$ that is too small will underestimate $g$ and overestimate mortality. For example, the Huso estimator (Huso, 2011; Huso and others, 2012) assumes $k=0$, which could lead to overestimating the total mortality. In some cases, though, the condition $k=0$ can be artificially approximated by disregarding carcasses that arrived prior to the search preceding the search in which they were discovered, reducing and potentially eliminating the bias. 


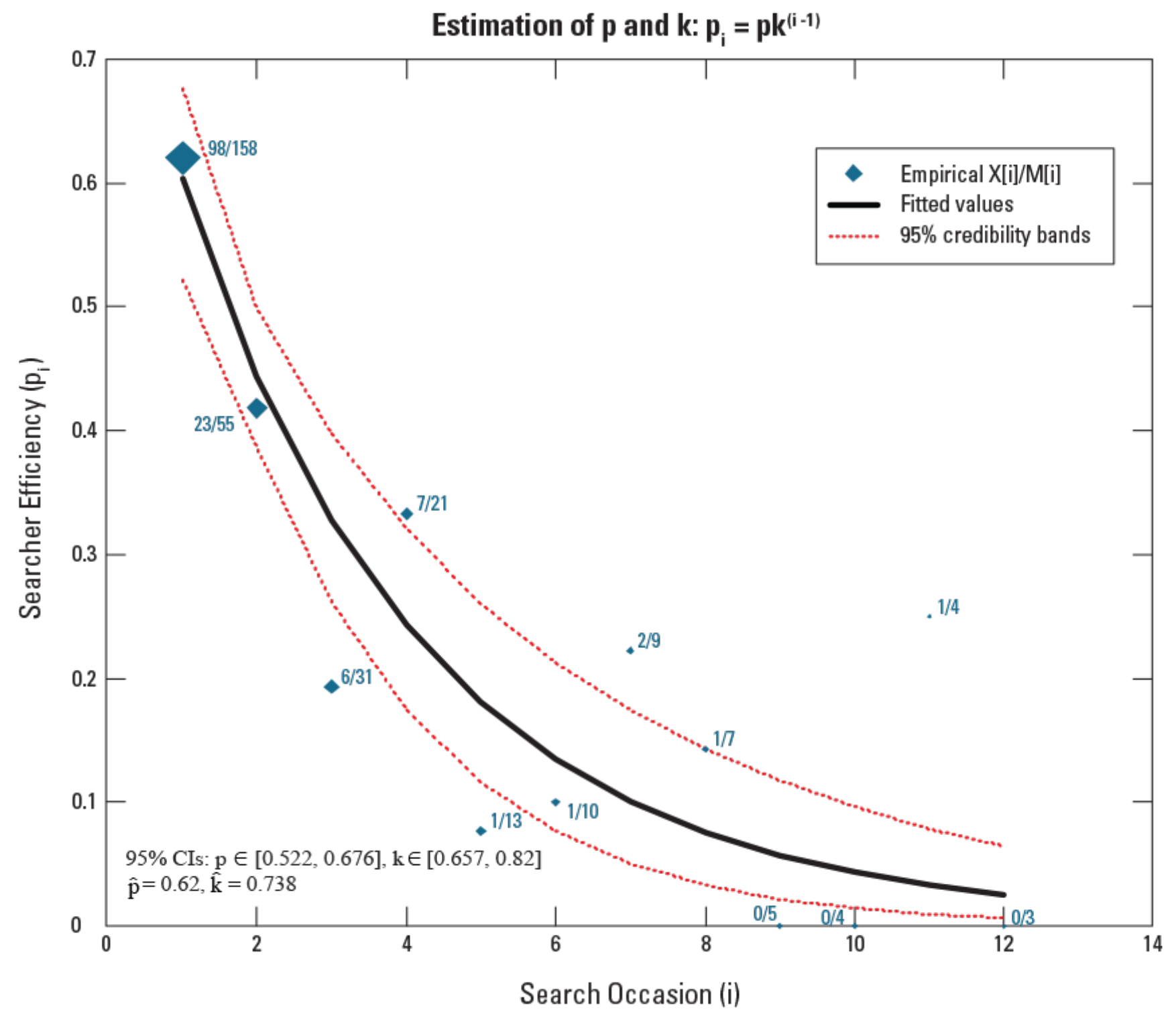

Figure 4. Graph generated by Evidence of Absence software showing searcher efficiency, search occasion, and estimation of $p$ and $k$. 


\subsubsection{Persistence Distribution}

The amount of time a carcass persists without being lost to scavenging or decay is modeled as a persistence distribution. EoA offers a choice of four persistence distributions - exponential, Weibull, log-logistic, or lognormal. The one-parameter exponential distribution makes a mathematically convenient assumption that the scavenging rate does not depend on age of carcass. In practice, the rate of carcass removal tends to change with time (Bispo and others, 2012; Warren-Hicks and others, 2013), and the exponential distribution usually does not accurately reflect the true persistence distribution. Frequently, it underestimates the scavenging rate for fresh carcasses and, as a consequence, underestimates the number of fatalities. The two-parameter Weibull distribution is a generalization of the exponential that offers more flexibility for modeling non-constant scavenging rates. When the scavenging rate is constant, Weibull and exponential are identical. In all other cases, the Weibull will be more accurate. Like the Weibull, the log-logistic and lognormal are two-parameter distributions that can accurately model a far wider array of persistence patterns than the exponential can. Typically, the Weibull, log-logistic, and lognormal models will produce persistence curves that are quite similar to each other, while the exponential curve will be notably different and substantially less accurate.

Persistence models can be entered into EoA either by fitting models to field trial data, or, if field trial data are not available or the user wishes to explore alternative scenarios, persistence parameters may be entered manually.

Parameterizations for the persistence distributions vary from author to author. EoA's parameterization largely follows those used by the base package in R but with some minor adjustments. The distributions are characterized by shape $(\alpha)$ and scale $(\beta)$ parameters. With the exponential distribution, the scale parameter is the mean carcass persistence time. The exponential is a singleparameter distribution, so no shape parameter is given. In its place, the exponential rate $(1 / \beta)$ representing the rate of carcass removal per unit time is given. Further details on the parameterizations can be found in appendix $\mathrm{H}$. 
The probability that a carcass persists until the first search after arrival is estimated as $r$. For example, a value of $r=0.685$ for $I_{r}=7$ implies that $68.5 \%$ of carcasses that arrive at a random time in an interval of 7 days will persist until the end of the interval. The value of $r$ is specific to an assumed search interval, $I_{r}$, with larger intervals associated with lower probability of persistence through the interval. The $r$ and $I_{r}$ parameters are not used in the estimation of total mortality or overall detection probability. They are provided strictly for user convenience as an aid in interpreting the fitted models. The value chosen for $I_{r}$ is the search interval $(I)$ if the search schedule has been entered by formula (that is, search interval and number of searches) or is the average length of the search intervals if a custom search schedule is used.

\section{Option: Use Field Trials to Estimate Parameters}

Clicking the View/Edit button opens the Carcass Persistence window, where the user may direct EoA to Get data from field trials. With carcass persistence trials, fresh carcasses are placed in the field periodically throughout the season, and each carcass location is checked on several occasions until it is no longer present or the study period ends. For example, carcasses may be checked 1, 2, 3, 4, $5,7,10,14,21$, and 28 days after being placed in the field. The latest time a carcass is known to be present is recorded as the minimum persistence time or CPmin. The earliest time a carcass is known to be missing is recorded as the maximum persistence time or CPmax. For carcasses that are still present at the end of the study, CPmin is the time of the last carcass check, and CPmax is entered as Inf (casesensitive). Field trial data are saved in a text file with comma-separated values (.csv). Format is two columns with case-sensitive headers (CPmin and CPmax) in the first row, and persistence times for carcasses are listed in the columns (table 1). Some researchers record persistence times in hours to increase the accuracy of the modeled persistence, especially for bats and small birds, which tend to have shorter persistence times than large birds, but persistence times should be converted to the same scale as the search schedule data before reading the data into EoA.

Table 1. Example format of carcass persistence data.

[CPmin represents the last time a carcass was observed. CPmax represents the time when a carcass was first noted as missing. Times are recorded in days elapsed since the carcass was placed in the field. For carcasses that are scavenged some time before the first carcass check, CPmin $=0$ and CPmax $=$ time of the first carcass check. For carcasses that are known to have been scavenged at an exact time (for example, a coyote is seen removing the carcass), $\mathrm{CPmin}=\mathrm{CPmax}=$ time of the scavenging event. For carcasses that are still present at the end of the field study, CPmin = time of final carcass check and CPmax $=\operatorname{Inf}($ which is case sensitive $)]$

\begin{tabular}{|c|c|}
\hline CPmin & CPmax \\
\hline 0.95 & 5.99 \\
\hline 4.93 & 6.85 \\
\hline 12.03 & 12.03 \\
\hline 0 & 3.11 \\
\hline 0 & 0.11 \\
\hline 22.1 & Inf \\
\hline 20.82 & Inf \\
\hline
\end{tabular}


After reading the .csv files, EoA fits exponential, Weibull, lognormal, and log-logistic persistence models to the field trial data, displaying the results in a table and graph (fig. 5). The empirical data are plotted as a Kaplan-Meier step function with 95\% CIs for persistence probabilities indicated by dotted lines (Kaplan and Meier, 1958). The fitted models are plotted as smooth curves, with colors corresponding to the distributions' colors in the table. For each distribution, the estimated shape $(\alpha)$ and scale $(\beta)$ parameters are given along with a $95 \%$ confidence interval for $\beta$.

The Akaike information criterion with correction for finite samples (AICc) is used to compare the four models (Burnham and Anderson, 2004). By itself, AICc has little practical value, but differences between AICc values of different models give an indication of the relative adequacy of the models for the given data. EoA gives the differences in AICc compared to the model with the smallest $\mathrm{AICc}$ as $\triangle \mathrm{AIC}$ and selects the model with the smallest AICc as the default but allows users to select a different model if desired. Models with $\Delta \mathrm{AIC}<2$ are considered comparable. Models with $\triangle \mathrm{AIC}>4$ have weak evidence to support them, and $\triangle \mathrm{AIC}>10 \mathrm{can}$ be interpreted as strong evidence that the model is not competitive with the best.

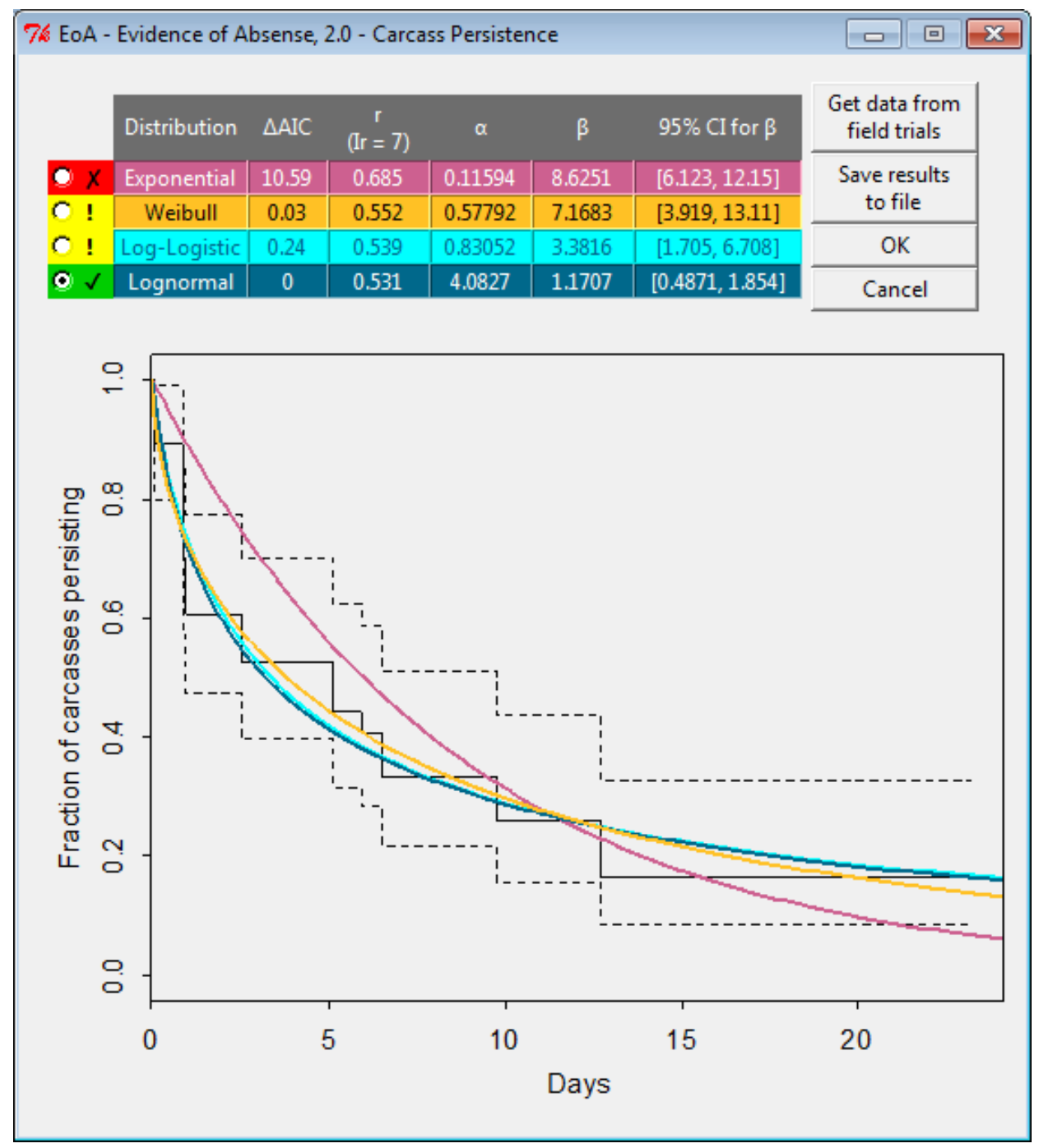

Figure 5. Screen capture of carcass persistence models. 
There are situations where a user may wish to enter persistence parameters manually rather than fitting models to persistence field trial data. One example would be if raw data from persistence trials are not available, but summary statistics from a previous analysis are. Another example would be if a user wished to explore scenarios with different persistence distributions. The user would choose the desired persistence distribution and then enter the shape $(\alpha)$ and scale $(\beta)$ parameters manually, along with a $95 \%$ confidence interval for the scale.

\subsection{Fatality Estimation $-M$ and $\lambda$}

The total mortality $(M)$ is estimated from the number of carcasses observed $(X)$ and the overall detection probability, which EoA estimates from the search data (appendix A). Mortality is estimated as $M^{*}$, which is tied to the user's choice of credibility level $(1-\alpha)$ and can be interpreted as a $100(1-$ $\alpha) \%$ upper bound on the number of fatalities or for statements like: "Based on the search data, we can assert with $100(1-\alpha) \%$ credibility that there were no more than $M^{*}$ fatalities." The credibility level $(1-\alpha)$ in Bayesian statistics is analogous to confidence level $(1-\alpha)$ in classical statistics.

The mortality rate parameter $(\lambda)$ is the average fatality event rate. For example, suppose a random process generates fatalities at an average 2.62 per year, then $\lambda=2.62$. In any given year, the actual number of fatalities will never be 2.62 but could readily be anything between 0 and 5 and would be very unlikely to exceed 8 . For a given set of search data, there will always be a degree of uncertainty about $\lambda$, even if detection probability is $100 \%$ and the number of fatalities in a year is known exactly. For example, if it is known that there were 3 fatalities in a year, those fatalities could have been generated from a process with mean of $\lambda=2.76$ or 6.31 or some other number near 3 , but it would be unlikely that the mean would be as large as $\lambda=9$ and highly unlikely to exceed $\lambda=15$. EoA expresses the uncertainty about $\lambda$ as a posterior distribution of $\lambda$ (appendix B), along with summary statistics, including mean, median, and $100(1-\alpha) \%$ CI.

\subsection{Single Class Module-Results}

EoA calculates estimates of detection probability $(g)$, total mortality $(M)$, and the mortality rate parameter $(\lambda)$ and provides graphical and text summaries of results.

\subsubsection{Estimate $g$}

Statistics for the estimated probability of detection are given in the Estimated detection probability ( $g$ ) results window (table 2) after the user clicks the Estimate $\boldsymbol{g}$ button. Statistics include a point estimate for $g$ along with a 95\% confidence interval (CI). Two statistics (Ba and $\mathrm{Bb}$ ) that are not as intuitive but are central to the mortality estimation also are given. $\mathrm{Ba}$ and $\mathrm{Bb}$ are parameter estimates of a beta distribution fit to parametric bootstrap values of $\hat{g}$ derived from the estimator equation (appendix A).

The probability of detecting a carcass that arrives in the monitored area during the monitored period is given as a restricted estimate of $g$. This estimate is then extrapolated to the full site but restricted to the monitored period. Finally, $g$ is estimated for the full site for the full year. 
Table 2. Results table generated by Evidence of Absence software showing estimation of $g$ in the Single Class Module.

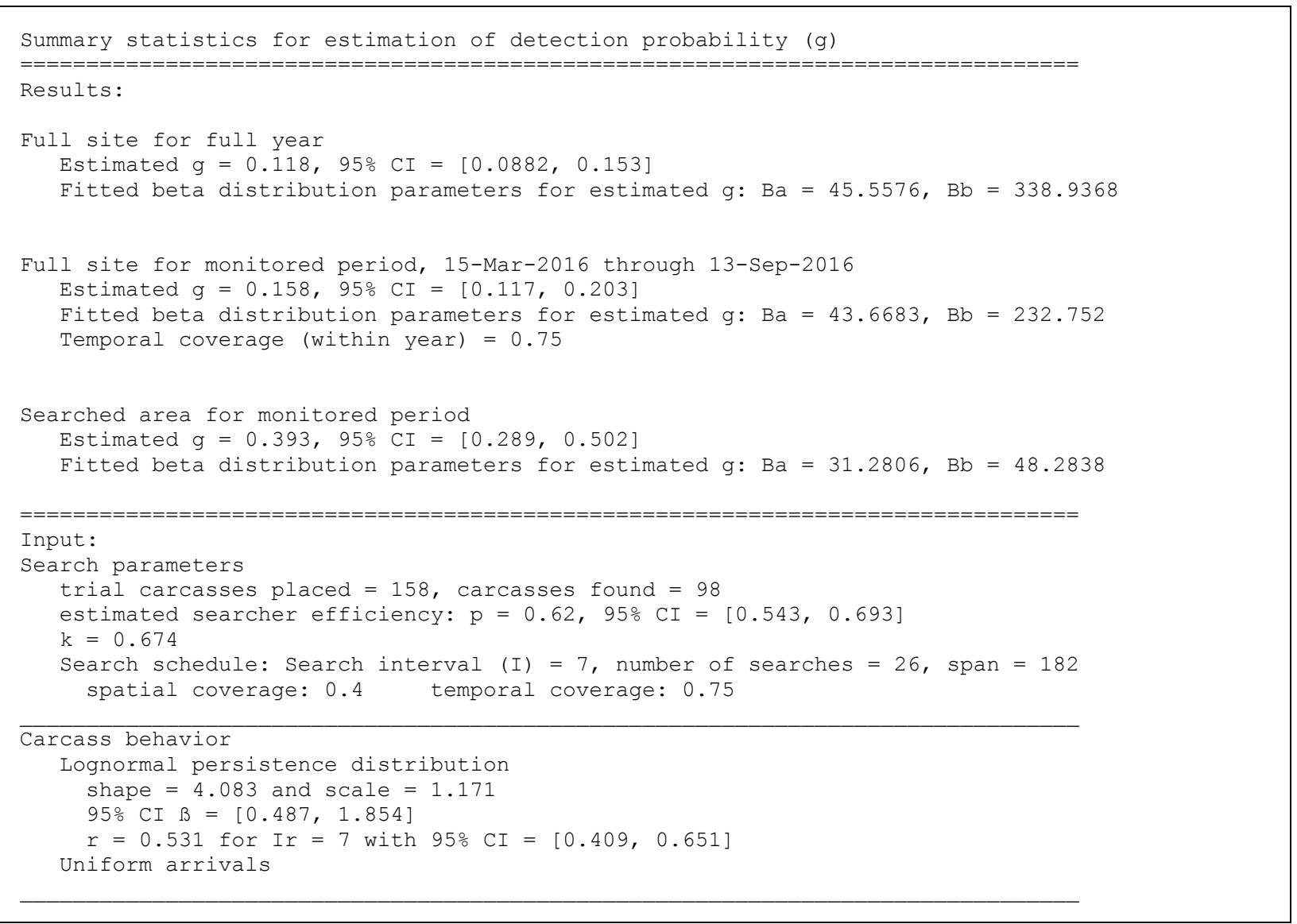

\subsubsection{Estimate $M$}

When the user clicks the Estimate $\boldsymbol{M}$ button, posterior distributions of $M$ and summary statistics for $M$ and for the detection probabilities are calculated and displayed both as a graph (fig. 6) and in tabular format (table 3). Finally, summary statistics for the search schedule and for the fitted searcher efficiency and carcass persistence models are given. If the option of a one-sided CI is selected, then the estimated mortality at a user-defined credibility level of $1-\alpha$ is given as $M^{*}$, which can be interpreted as the $100(1-\alpha) \%$ credible upper bound for fatality. For example, in the full year results for the default Single Class data set (fig. 6), $M^{*}=39$ for $1-\alpha=0.9$ means that the probability that the true number of fatalities was less than or equal to 39 is at least $90 \%$ according to posterior distribution. For $1-\alpha=0.5, M^{*}=18$ is a balanced point estimate of $M$ with the odds that $M>M^{*}$ and odds that $M \leq M^{*}$ being about equal. Larger values of $1-\alpha$ yield larger $M^{*}$ 's with greater assurance that $M \leq M^{*}$. If the option of a two-sided CI is selected, then the estimated mortality is given as a $100(1-\alpha) \%$ credible interval (CI) for $M$ along with the median of the posterior.

The posterior distributions of $M$ are given in both graphical and tabular format for total mortality at the entire site for the full year and for the entire site during the monitored period. In addition, a table of the posterior distribution of $M$ for fatalities arriving only in the monitored area during the monitored period is given in the text summary of results. 

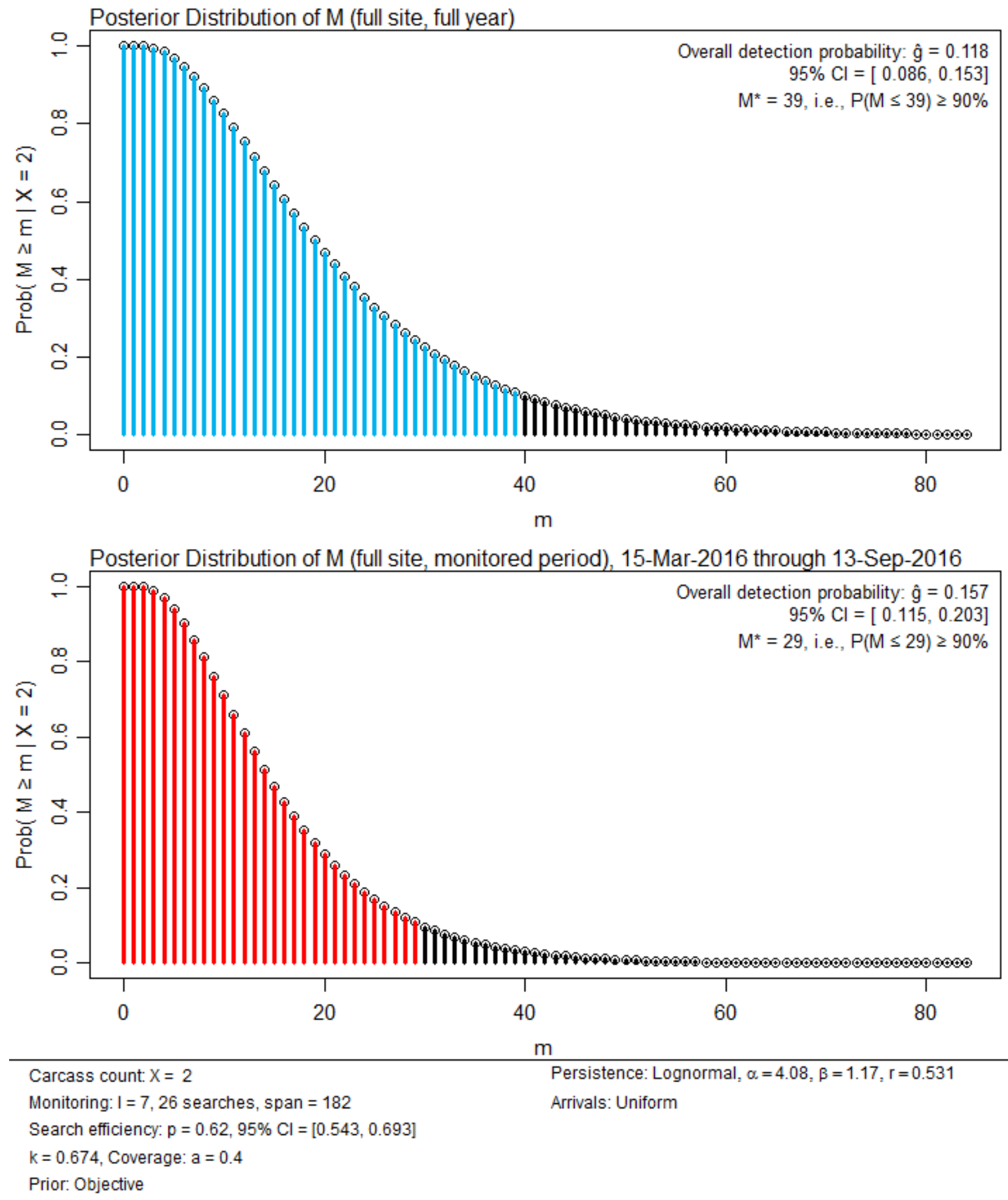

Figure 6. Graphs generated by Evidence of Absence software showing estimation of $M$ in the Single Class Module (graphical results for $M^{*}$ ). 
Table 3. Results table generated by Evidence of Absence software showing estimation of $M$ in the Single Class Module (tabular results for $M^{*}$ ).

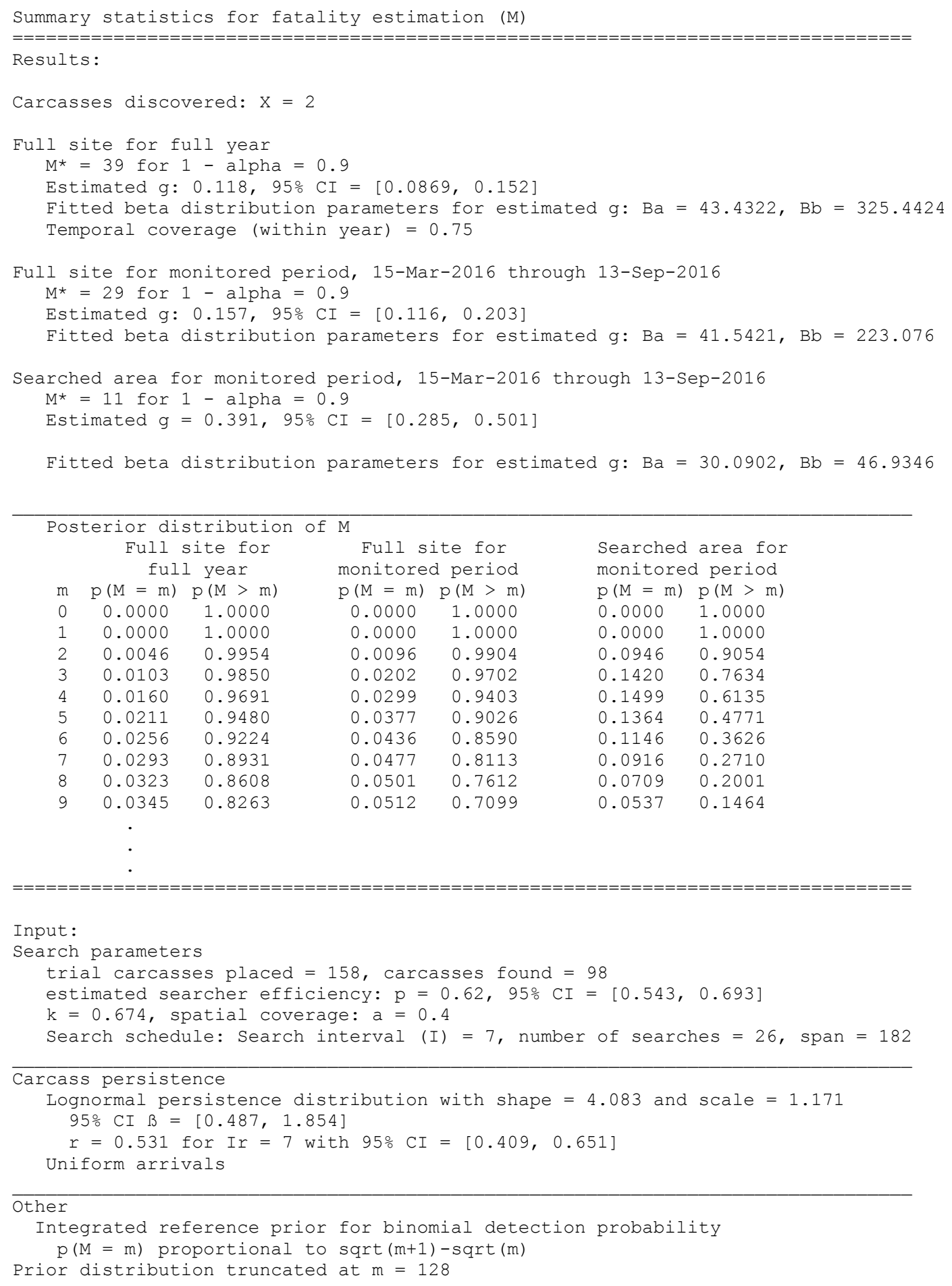


If the option of a one-sided CI is selected, graphs of the posterior distributions display the complementary cumulative distribution functions ( $1-\mathrm{CDF}$ or CCDF). That is, for a given number of fatalities $m$ on the $x$-axis, the height of the bar corresponds to the probability that the number of fatalities was at least $m$. For example, in the default data set $X=2$ carcasses were observed, so there is $100 \%$ probability that the number of fatalities was at least two. Correspondingly, the bars at $m=$ 0,1 , and 2 extend to 1.0 on the $y$-axis (fig. 6). At the other extreme, given that only two carcasses were discovered and the detection probability for the full year was $\hat{g}=0.118$, it is highly unlikely that there were more than 60 fatalities. This is reflected in the very short bar at $m=60$ (fig. 6 , top graph). The colored region represents values of $M \leq M^{*}$, which is the one-sided $100(1-\alpha) \%$ CI for $M$. In most cases, the probability that $M$ exceeds $X / \hat{g}$ is about $50 \%$, as reflected in this example at values of $m$ near $X / \hat{g}=\frac{2}{0.118}=16.9$ with bar heights near 0.5 (that is, $P(M \geq 16.9) \approx 0.5$ ) in the top graph in figure 6 . However, this is only a back-of-the-envelope approximation that does not account for uncertainty in $\hat{g}$, and $X / \hat{g}$ frequently underestimates the median, especially when $g$ and $X$ are small or when there is great uncertainty in the estimate of $g$. Compared with the posterior restricted to the monitored period, the full-year posterior appears to be pushed farther to the right to account for the fatalities that could have occurred outside the monitored period (fig. 6).

If the option of a two-sided CI is selected, graphs of the posterior distributions display the probability density functions (PDFs). That is, for a given number of fatalities $m$ on the $x$-axis, the height of the bar corresponds to the posterior probability that the number of fatalities was equal to $m$. For example, in the default data set $X=2$ carcasses were observed, so there is $0 \%$ probability that the number of fatalities was less than two and there are no bars at $m=0$ and 1 (fig. 7). The colored regions represent the nominal $100(1-\alpha) \%$ CI for $M$. More precisely, the posterior probability that $M$ is in the $\mathrm{CI}$ is at least $1-\alpha$, and the probability that $M$ lies to the left of the interval is no more than $\alpha / 2$. Note that the posteriors tend to be right-skewed (especially when $\hat{g}$ is small or has large variance), so that the mean and $X / \hat{g}$ will typically be located somewhat to the right of the peak.

The posterior distributions of $M \mid(X, \hat{g})$ are reproduced as both PDFs and CCDFs in a table in the results window (table 3 ). In addition, for advanced users, $\mathrm{R}$ functions are provided for calculating posterior distributions of $M(\mathrm{PDF}), M^{*}$ s, and CIs given $X$ and $\hat{g}$ (or $g$ ) (table 4). For example, the functions postm. ab (x, $\mathrm{Ba}, \mathrm{Bb})$ and $\operatorname{postm}(\mathrm{x}, \mathrm{g})$ return the posterior distribution of $M$ conditioned on carcass count and detection probability (as characterized by beta distribution parameters $B a$ and $B b$ when $g$ is estimated as $g \sim \operatorname{beta}(B a, B b)$ or simply as $g$ if $g$ is known with certainty). 

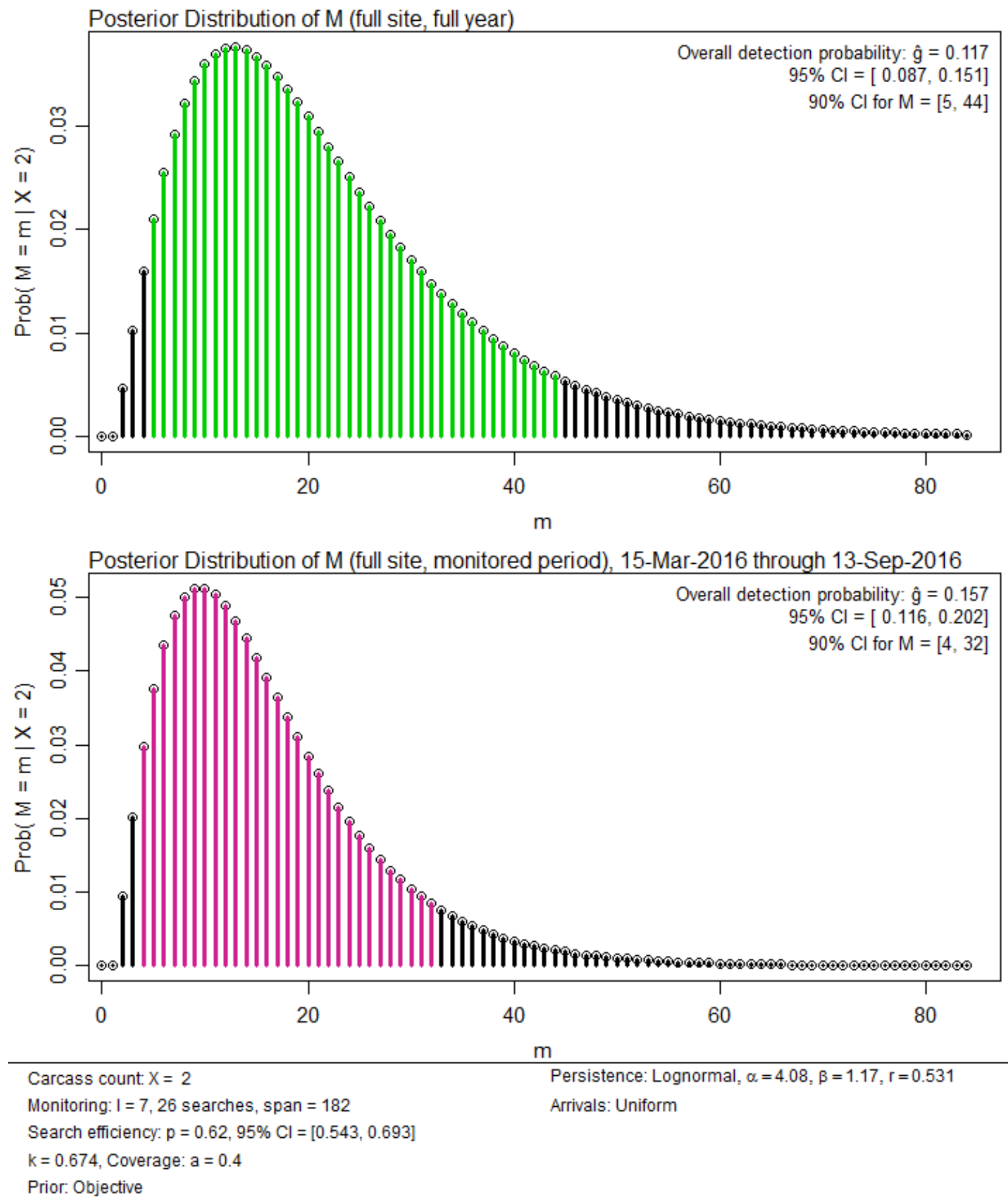

Figure 7. Graphs generated by Evidence of Absence software showing estimation of $M$ in the Single Class Module (graphical results for two-sided credible interval). 
Table 4. R command line functions for calculating key Single Class results manually.

[Inputs include $\mathrm{x}=$ carcass count and the detection probability, which may be entered either as beta distribution parameters, $\mathrm{Ba}$ and $\mathrm{Bb}$ (when $g$ is estimated), or as $g$ (when $g$ is assumed to be fixed and known). The posterior $\mathrm{L}$ family of functions return functions rather than arrays. Additional functions and data accessible from the command line are provided in appendix I]

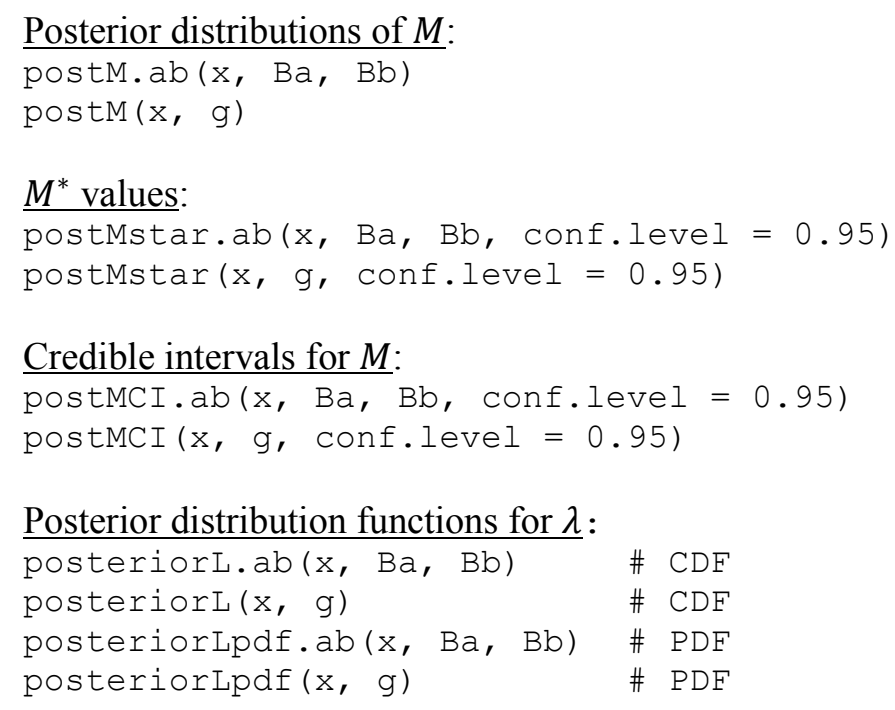

\subsubsection{Estimate $\lambda$}

The posterior distribution of the estimated mortality rate parameter $(\lambda)$ given the carcass count $(X)$ and the estimated detection probability $(\hat{g})$ is calculated and displayed in a graph as a CDF along with the mean, median, 5th and 95th percentiles, $100(1-\alpha) \%$ CI for $\lambda$, and summary statistics for detection probability (fig. 8).

For advanced users, the posterior distributions (CDFs) of $\lambda \mid(X, \hat{g})$ and $\lambda \mid(X, g)$ also are provided as $\mathrm{R}$ functions posteriorL.ab ( $\mathrm{x}, \mathrm{Ba}, \mathrm{Bb})$ and posteriorL $(\mathrm{x}, \mathrm{g})$, respectively (table 4). For example, the posterior distribution of $\lambda \mid(X=2, g=0.117)$ can be defined and used as follows:

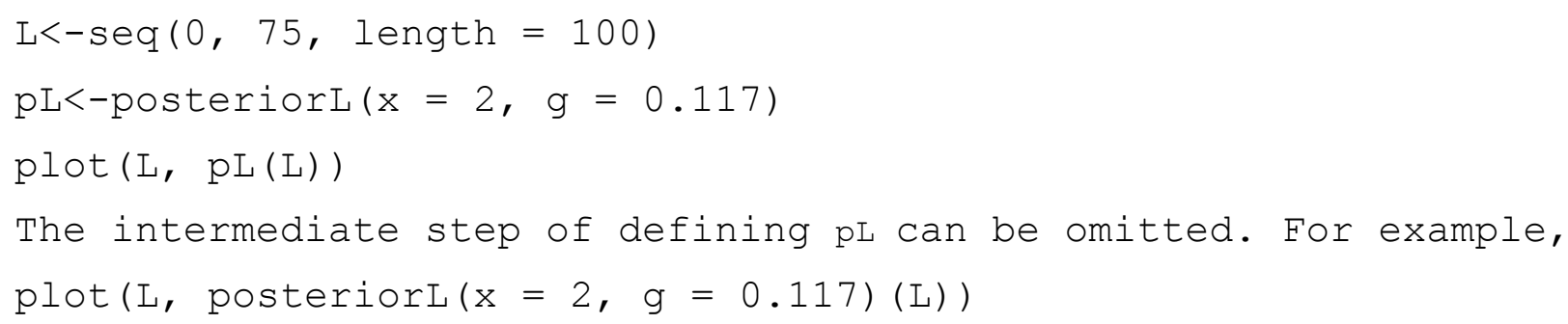

Similarly, the posterior PDFs are also provided as R functions posteriorLpdf.ab ( $\mathrm{x}, \mathrm{Ba}, \mathrm{Bb})$ and posteriorLpdf $(x, g)$, with implementation like that of the CDFs.

In addition, $\mathrm{R}$ functions are provided for calculating CIs for $\lambda \mid(X, \hat{g})$ and $\lambda \mid(X, g)$ for userdefined $1-\alpha$ credibility level, namely postL.abCI (x, Ba, Bb, conf.lev $=0.95$ ) and postl.CI $(x, g, \operatorname{conf} .1 \mathrm{ev}=0.95)$, respectively. More functions and data that are accessible from the command line are described in appendix I. 


\section{Posterior of $\lambda$}

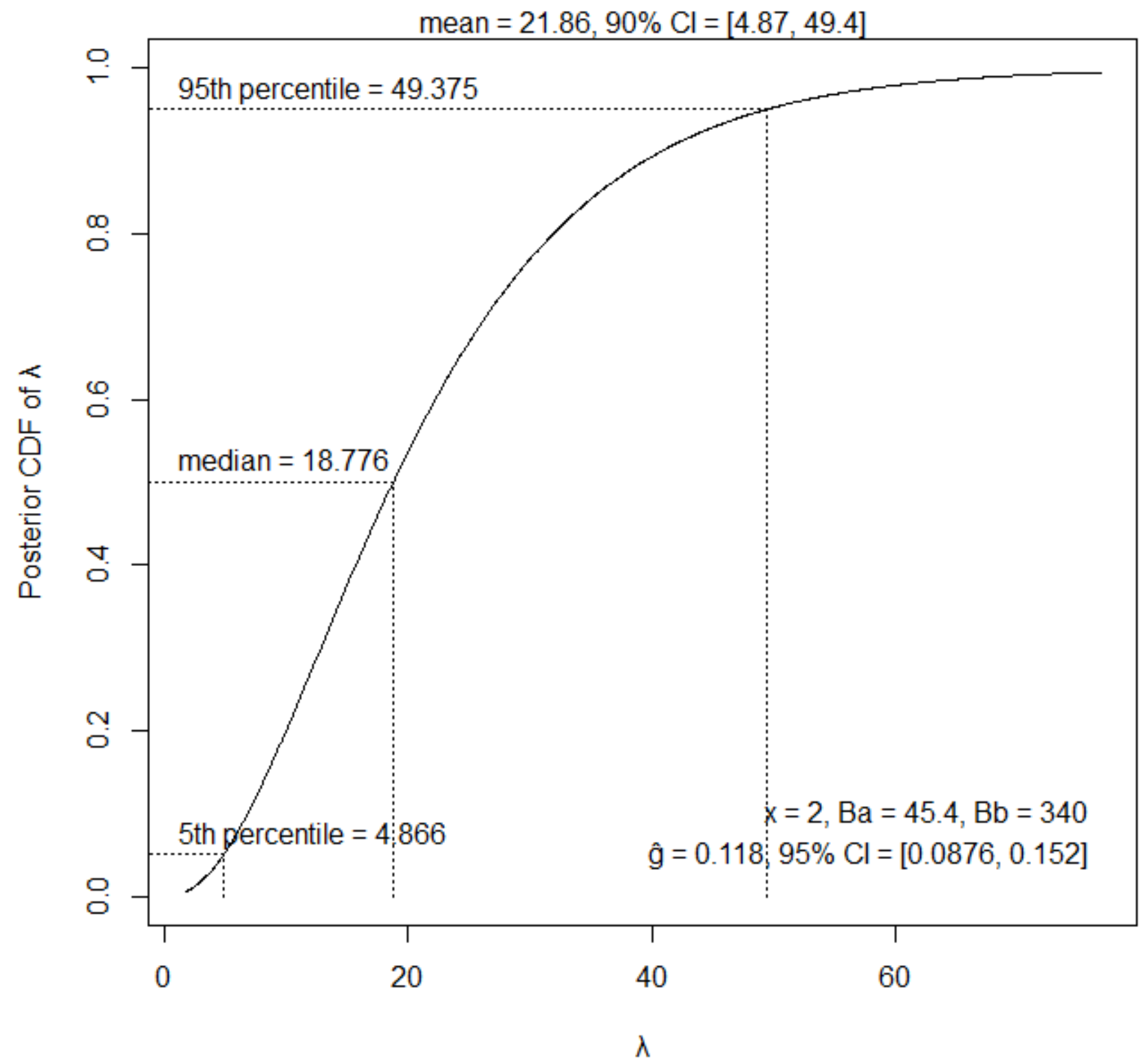

Figure 8. Graph generated by Evidence of Absence software showing posterior distribution of $\lambda$ for entire site for full year, Single Class Module. 


\section{Multiple Class Module}

When the searched area is divided into two or more classes or subunits, the Multiple Class Module can be used to combine data from independent classes to estimate the total mortality. For example, a given site may be subdivided into several classes depending on vegetative cover (for example, easy, moderate, and difficult visibility), ground texture or color (for example, roads and pads versus cleared ground), or season (for example, spring and fall). Also, different sites within a region may each be taken as separate classes and combined to estimate a regional total.

\subsection{Data and Parameters}

Data from each class are analyzed separately, and summary statistics are entered into the class table (fig. 9). The coverage $\left(a_{\mathrm{i}}\right)$ for class $i$ is the fraction of the total carcasses arriving a given class (Huso and Dalthorp, 2014), and the values in the $a$ column must sum to 1.0. $X$ is the observed number of carcasses. $B a$ and $B b$ are the parameters of the beta distribution characterizing $\hat{g}$. The $\hat{g}$ and $95 \% \mathrm{CI}$ columns are point and interval estimates of the overall detection probabilities for each class. These are a function of $B a$ and $B b$ and cannot be edited directly by the user.

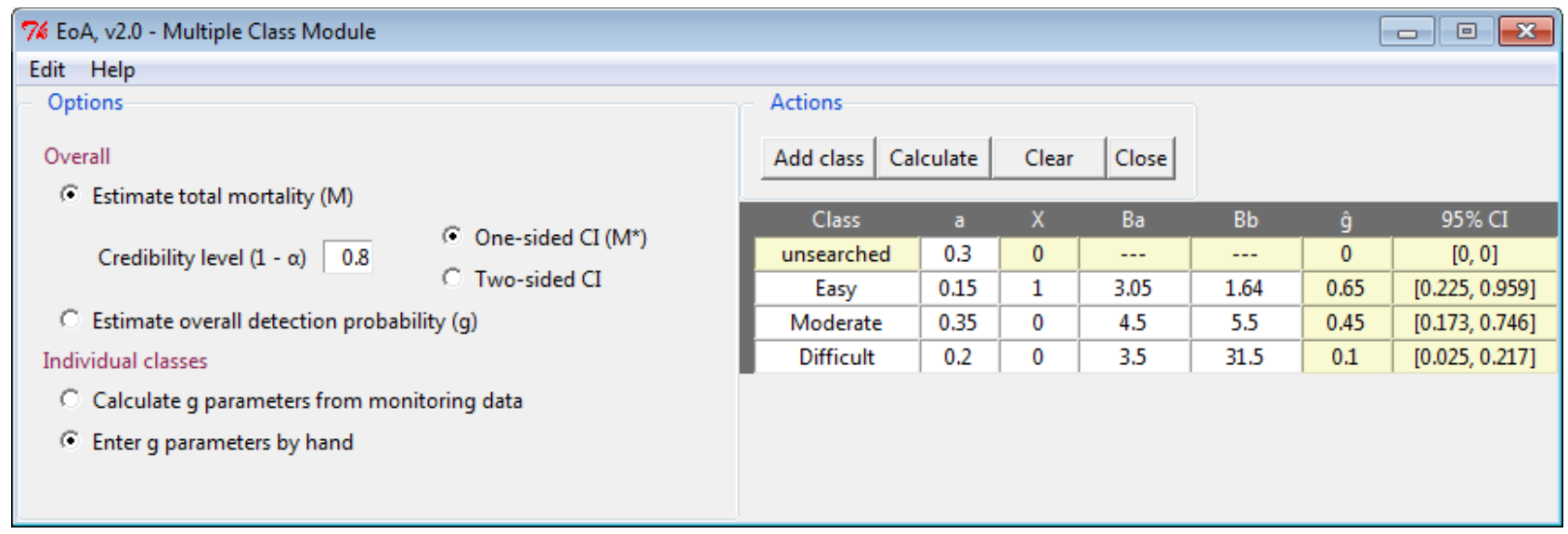

Figure 9. Screen capture of Multiple Class Module (inputs).

\subsubsection{Overview of $B a$ and $B b$ Parameters}

The $B a$ and $B b$ parameters characterize the estimated detection probability $(\hat{g})$ along with its uncertainty, or, more precisely, $\hat{g} \sim \operatorname{beta}(B a, B b)$. From $B a$ and $B b$, myriad statistics about the detection probability can be calculated. For example, the expected value of $\hat{g}$ is $\mathrm{E}[\hat{g}]=\frac{B a}{B a+B b}$, which is a good point estimate of $g$; the variance of $\hat{g}$ is $\mathrm{V}[\hat{g}]=\frac{B a \cdot B b}{(B a+B b)^{2}(B a+B b+1)}$, which gives a good indication of the degree of uncertainty associated with the estimated $g$; and a $95 \%$ CI for $g$ would be $\left[F^{-1}(0.025 ; B a, B b), F^{-1}(0.975 ; B a, B b)\right]$ where $F(x ; B a, B b)$ is the CDF of the beta distribution with parameters $B a$ and $B b$, and $F^{-1}$ can be calculated in $\mathrm{R}$ as qbeta (x, shape1 = Ba, shape2 $\left.=\mathrm{Bb}\right)$. 
Crucial to the interpretation of the $B a$ and $B b$ parameters and the estimated detection probabilities that they characterize is that they are specific to a particular period of inference, and the $B a$ 's and $B b$ 's listed in the input table in the Multiple Class Module are assumed to apply to the same period of inference. For example, suppose carcasses arrive uniformly through the year at a site that is subdivided into two search classes.

\subsubsection{Calculating $B a$ and $B b$ Parameters}

There are several options for calculating the $B a$ and $B b$ values to enter into the table. One is to select the Calculate $g$ parameters from monitoring data option and click the Add class button. This will open the Search Class window for entering search data as with the Single Class Module (fig. 2) but with two minor differences. First, it is assumed that there is $100 \%$ search coverage within the class, and thus the class coverage $\left(a_{\mathrm{i}}\right)$ is ignored in the calculation of $B a$ and $B b$. Second, when the Estimate $\boldsymbol{g}$ or Estimate $\boldsymbol{M}$ button is clicked, the data entry form closes, and the resulting estimated $B a$ and $B b$ parameters are entered into the class table in the Multiple Class Module. $B a$ and $B b$ parameters for each class can be successively calculated in this way to fill in the class table. Care must be taken to ensure that the period of inference remains the same for all classes. For example, if the monitoring period varies among classes, then the temporal coverage, class names, and $\left(a_{\mathrm{i}}\right)$ parameters must be entered manually.

Care must be taken to ensure that the period of inference is the same for all classes. For example, if the monitoring period is the same for all classes, the temporal coverage parameter $(v)$ in the Search Class window should be set to the same value for all classes, which would typically be: (1) $v=1.0$ in order to restrict inference to the monitored period or (2) $v$ equal to the fraction of annual fatalities that are expected to occur during the monitored period in order to define the period of inference as the full year.

A second option is to select Enter $g$ parameters manually and calculate $B a$ and $B b$ for each class using the Single Class Module with search coverage set to $a=1$. After calculating $B a$ and $B b$, the values may be recorded separately and entered into the multiple class table manually.

Finally, if original search data are not available but estimates of $\hat{g}$ are, the estimated detection probabilities (with $95 \%$ CIs) can be converted to corresponding $B a$ and $B b$ parameters $^{3}$ using the Parameter Conversion Calculator which can be opened from the Help menu. For example, when $\hat{g}=0.2$ with $95 \% \mathrm{CI}=[0.1,0.3]$ are entered, the corresponding $B a$ and $B b$ parameters are automatically updated (fig. 10). The values can then be recorded and entered manually into the multiple class table.

\footnotetext{
${ }^{3}$ Normally, there is no beta distribution with $B a$ and $B b$ parameters that give a mean of $\hat{g}$ and the given $95 \%$ CI, so an approximation must be used. The parameter conversion calculator gives the $B a$ and $B b$ parameters that define a beta distribution with mean $=\hat{g}$ and standard deviation $=1 / 4$ of width of the $95 \% \mathrm{CI}$.
} 


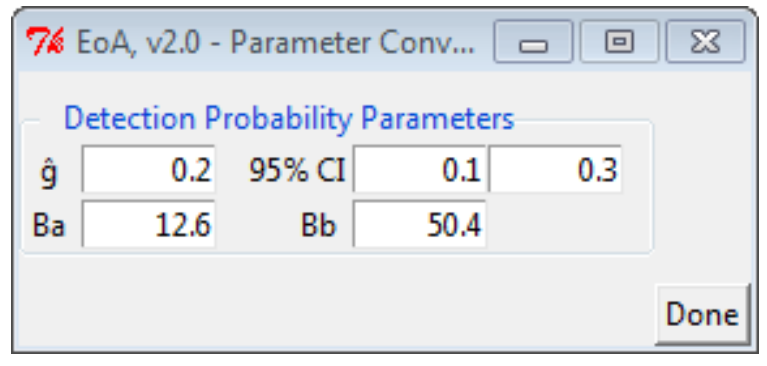

Figure 10. Screen capture of parameter conversions calculator. For a given $\hat{g}$ with $95 \% \mathrm{Cl}$, the calculator gives $\mathrm{Ba}$ and $B b$ parameters of a beta distribution with mean $=\hat{g}$ and with 0.025 and 0.975 quantiles approximately equal to the given bounds on the $\mathrm{Cl}$ for $\hat{g}$. Conversely, for given $B a$ and $B b$, the calculator gives $\hat{g}=$ mean and bounds on the $95 \% \mathrm{Cl}$ equal to the 0.025 and 0.975 quantiles of a beta distribution with parameters $B a$ and $B b$.

\subsection{Multiple Class Module-Results}

After the data have been entered, the posterior distribution of $M$ and summary statistics are presented in both graphical and tabular format upon clicking the Calculate button. The results graphs are similar to the results graphs for the Single Class (section 2.3.2) with one key difference: the results for Single Class show posterior distributions of $M$ for two different periods of inference (monitored period and full year), whereas results graphs for Multiple Classes show posterior distributions of $M$ only for the common period of inference among the separate classes.

Like the results table for Single Class (table 3), the results table for Multiple Classes gives summary statistics for $\hat{g}$ and $M^{*}$ but restricted to the common period of inference among the classes (table 5). In addition, there is a likelihood ratio test of the plausibility of the assumed relative weights (as discussed in appendix F, section F.3). Misspecification of the relative weights in the multiple class model can introduce bias, especially when the detection probabilities vary considerably among classes. There is a tradeoff between bias and variance in mortality estimators. The EoA method of combining data from search classes introduces some potential for bias in exchange for a substantial reduction in variance. When counts are small, that tradeoff results in smaller errors on average for EoA than for the often-used Horvitz-Thompson estimator (appendix E). 
Table 5. Results table generated by Evidence of Absence software showing Multiple Class estimates (posterior distribution of $M$ has been truncated to save space).

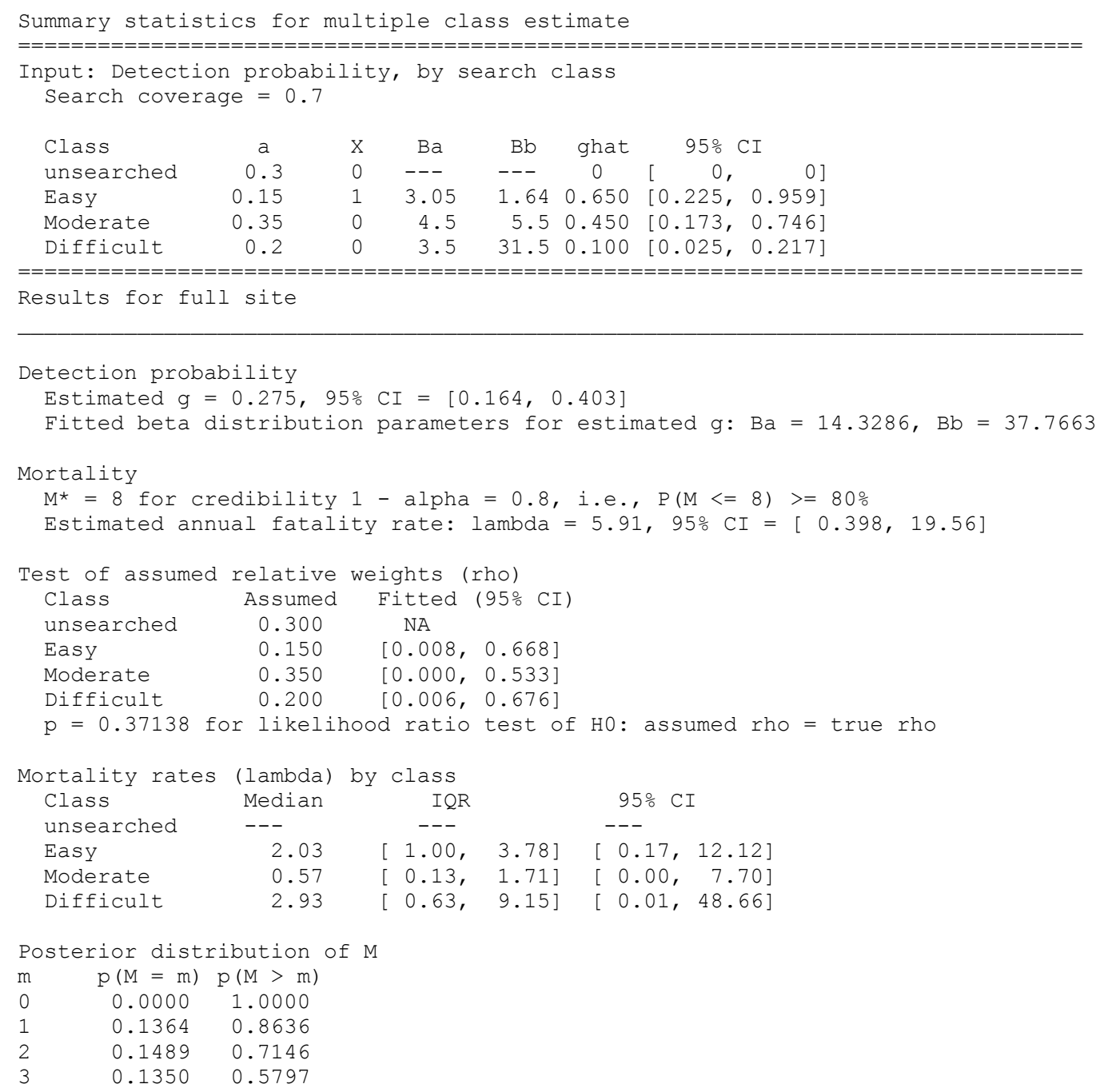

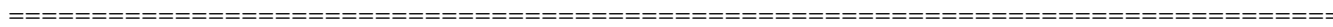




\section{Multiple Years Module}

The Multiple Years Module (fig. 11) provides tools for tracking estimated fatalities and mortality rates in the past and for projecting future mortality $(M, \lambda)$ and mortality estimates $\left(M^{*}, \hat{\lambda}\right.$, and their credible intervals).

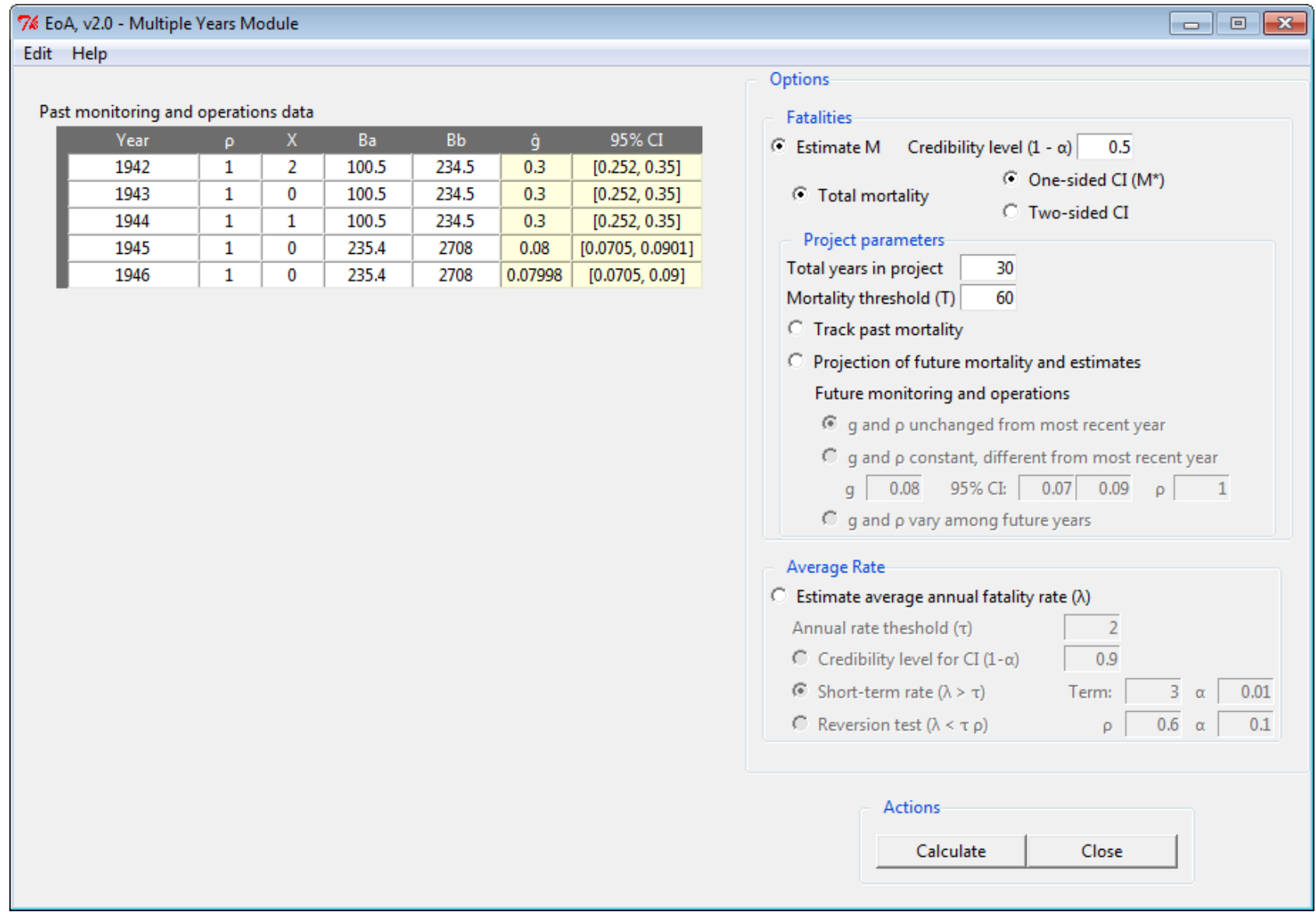

Figure 11. Screen capture of Multiple Years Module (inputs). 


\subsection{Past Monitoring and Operations Data}

In the Past monitoring and operations data table (fig. 11), for each year monitored, the user enters the year, the relative mortality rate $(\rho)$, the number of carcasses observed $(X)$, and the $B a$ and $B b$ parameters for the detection probability.

Data can be entered manually, by importing from a previously saved .rds file, or by copy-andpaste from a text file or spreadsheet. New rows can be appended to the bottom of the table using the keyboard shortcut $<\mathrm{Ctrl}+\mathrm{a}>$ or by pressing $<$ Enter $>$ when the cursor is in the last row of the table. Similarly, the active row can be deleted using $<\mathrm{Ctrl}+\mathrm{d}>$. Data in the " $\hat{g}$ " and " $95 \% \mathrm{CI}$ " columns are calculated from $B a$ and $B b$ and cannot be edited directly manually. Also, the table cannot be edited when the Projection of future mortality and estimates option is selected.

\subsubsection{Year}

The model regards the Year column as nothing more than a list of names for independent monitoring periods. In many cases, the Year column refers to a calendar year, but each line could be any period of time, for example a season or a phase of a project, and should be understood as such, although for convenience we refer to it as a year. The model does assume that the years are independent, that is, the estimated detection probabilities are based on separate carcass persistence and searcher efficiency trials each year.

\subsubsection{Relative Mortality Rate $(\rho)$}

The assumed relative mortality rate is $\rho$. If there are no changes in operations and no reason to suspect mortality rates varied systematically from year to year, then $\rho=1$ each year. However, if operations or ecological conditions change, the $\rho$ parameter should be adjusted to reflect changes. For example, if a site is expanded by $20 \%$ in year 3 , then $\rho=1$ for years 1 and 2 as a baseline and $\rho=1.2$ in year 3 would be appropriate. Or if minimization measures that are expected to reduce fatalities by $30 \%$ are implemented in year 3 , then $\rho=1$ for years 1 and 2 , and $\rho=0.7$ for year 3 .

\subsubsection{Number of Observed Carcasses $(X)$}

The number of carcasses that have been observed each year is expected to be small $\left(X_{i} \leq 5\right)$. If the number of carcasses is large $\left(X_{i} \geq 10\right)$, the model is still valid but may be more sensitive to potential bias if the relative weights $\left(\rho_{i}\right)$ are misspecified.

\subsubsection{Detection Probability Parameters $(B a$ and $B b)$}

The detection probability parameters for each year are calculated using EoA's Single Class Module with the monitoring data from the year in question, recorded, and then entered into the Past monitoring and operations data table. An overall detection probability for combined years is then calculated from the yearly $B a$ and $B b$ parameters and the relative weights, $\rho$ (appendix F). 


\subsection{Options}

The user has the option of estimating the actual number of fatalities $(M)$ or the average fatality rate $(\lambda)$, as described in section 2.2 .

\subsubsection{Estimate $M$}

Mortality estimates are defined for a specific credibility level, $1-\alpha$, which is entered by the user. The value of $1-\alpha$ can be interpreted as approximately the probability that the true number of fatalities $(M)$ is less than or equal to the estimated number of fatalities $\left(M^{*}\right)$. An $M^{*}$ based on a credibility level of $1-\alpha=0.5$ is the most accurate (in the sense that odds are about 50-50 that $M \leq M^{*}$ ), while higher credibility levels yield larger $M^{*}$ and greater assurance that $M \leq M^{*}$.

The option allows the user to estimate Total mortality in past years, Track past mortality (both annual and cumulative), or calculate a Projection of future mortality and estimates.

\subsubsection{Total Mortality}

Under the "total mortality" option, the posterior distribution of the total mortality over the period of time spanned by the search data is calculated. If the option of a one-sided CI is selected, then the estimated mortality at a user-defined credibility level of $1-\alpha$ is given as $M^{*}$, which can be interpreted as the $100(1-\alpha) \%$ credible upper bound for fatality. If the option of a two-sided CI is selected, then the estimated mortality is given as a $100(1-\alpha) \%$ credible interval $(\mathrm{CI})$ for $M$ along with the median of the posterior.

Results are displayed in both graphical (fig. 12) and text format (table 6). The overall detection probability $(g)$ is the probability of finding a carcass that arrives at the site at any time during the monitored years. The baseline fatality rate $(\lambda)$ is the fatality rate in a year with relative weight of $\rho=1$. In general, the fatality rate in year $i$ is $\rho_{i} \lambda$. Point and interval estimates of both $g$ and $\lambda$ are given. For example, with the default multiple years data set, the total number of fatalities in the 5 years is estimated to be $M^{*}=15$ for $1-\alpha=0.5$, which is a point estimate of the true number of fatalities. A precise interpretation would be that the probability that the true number of fatalities was less than or equal to 15 is at least $50 \%$ according to the posterior distribution.

To some degree, estimates of multi-year total mortality depend on the relative weights $\left(\rho_{i}\right)$ as defined by the user. However, in most cases the EoA model is robust with respect to assumptions about $\rho_{i}$ 's, and mortality estimates are often largely unaffected by misspecification of $\rho_{i}$ 's. In some cases, though, misspecification of the $\rho_{i}$ 's can significantly influence mortality estimates. EoA tests whether the monitoring data provide evidence that the $\rho_{i}$ 's are significantly misspecified and the degree to which that misspecification may introduce bias into the mortality estimates. 


\section{Posterior Distribution of Total Fatalities over 5 years}

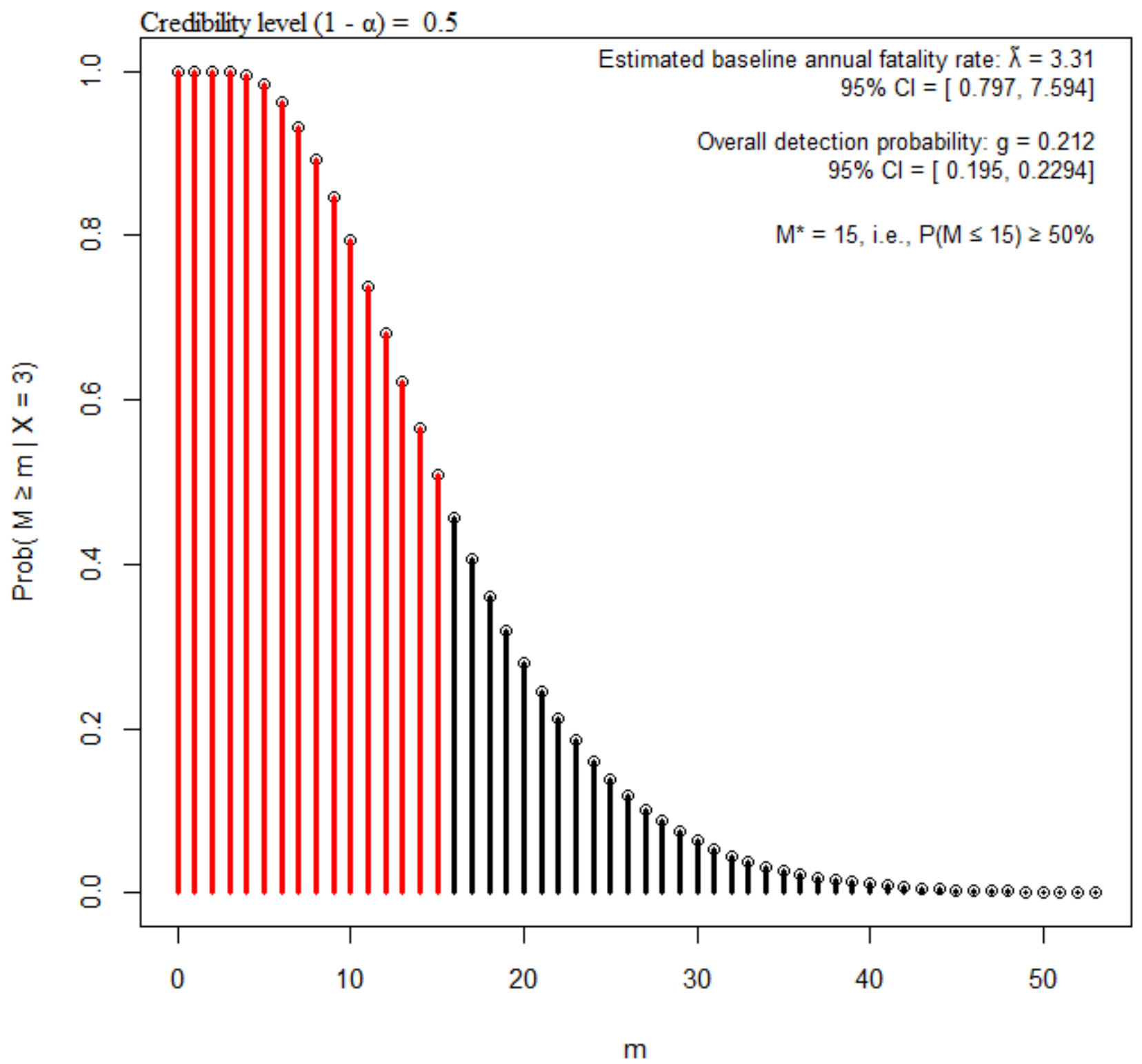

Figure 12. Graph generated by Evidence of Absence software showing posterior distribution of $M$ (Multiple Years Module). 
Table 6. Results table generated by Evidence of Absence software showing estimation of total mortality in Multiple Years Module.

[The original posterior distribution of $M$ has been truncated in the table to save space]

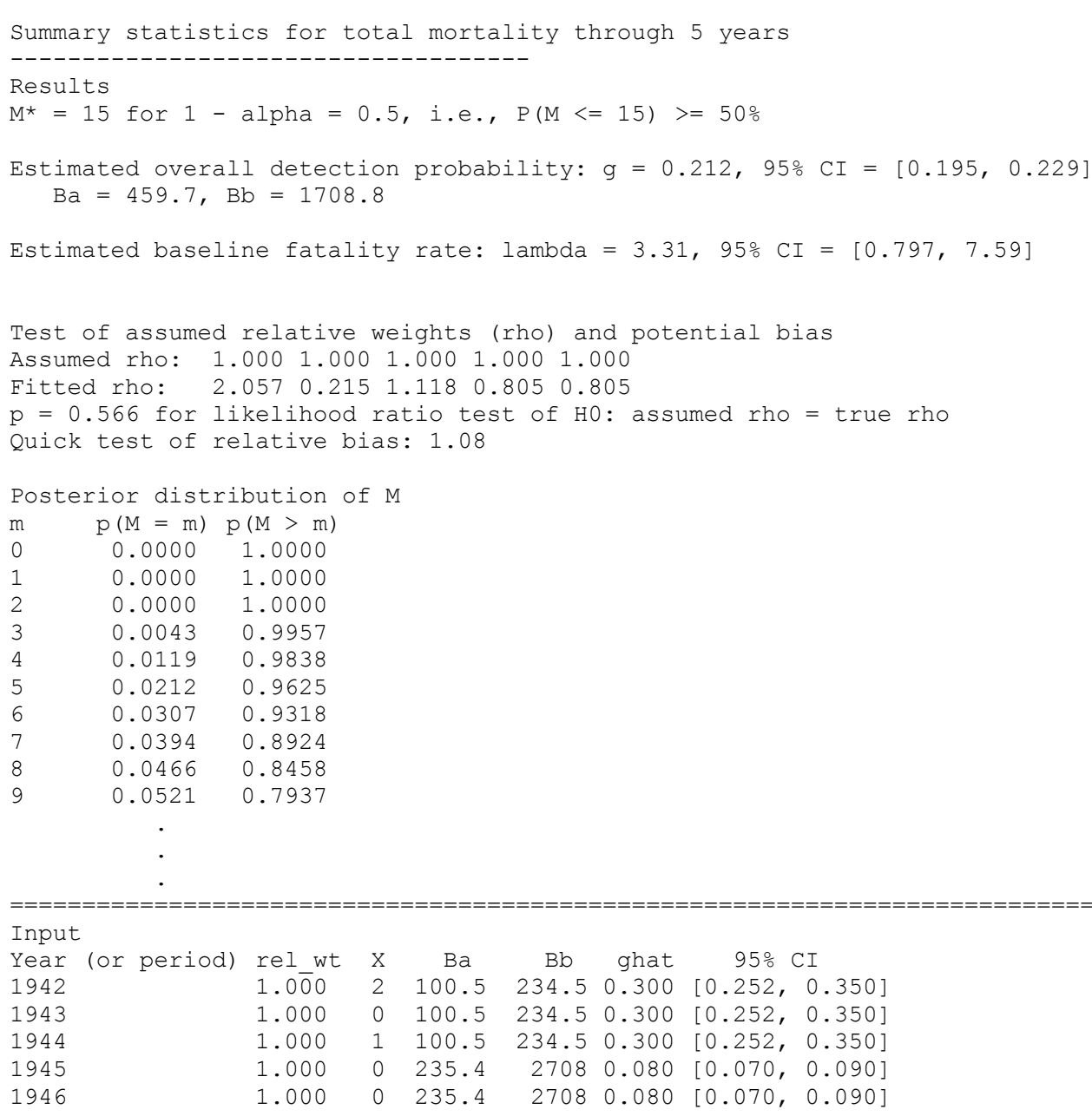


Results of statistical tests (appendix F, section F.3) of the plausibility of the assumed relative weights $(\rho)$ are included in the text output (table 6). The assumed values of $\rho$ for each year are the values entered by the user in the Past monitoring and operations data table in the Multiple Years Module window. The first test is a simple, side-by-side comparison of the assumed $\rho$ values versus the fitted $\rho$ values, which represent estimates of the true relative weights as calculated from the search data (section G.3.1). This simple test gives a qualitative indication of the degree to which the assumed $\rho$ 's may be misspecified. However, typically there is a high degree of uncertainty in the fitted $\rho$ 's, so a quantitative significance test also is performed. Taking into account the uncertainty in fitted $\rho$ 's, a $p$ value is calculated for a likelihood ratio (LRT) test of whether the assumed $\rho$ 's are equal to the true $\rho$ 's (section G.3.2). When detection probabilities do not vary from year to year, $\rho$ has no effect on mortality estimates, and no bias is introduced even if the assumed $\rho$ 's are badly mistaken. However, if $\hat{g}$ varies markedly from year to year and the $X_{i}$ 's are not small, then mortality estimates may be sensitive to the assumed $\rho$ values, introducing bias into the mortality estimates. Thus, significant misspecification of $\rho$ 's is a cause for concern, although it may not have a significant effect on fatality estimates. A quick test for bias (section G.3.3) helps inform interpretation of the LRT. The ratio of the $1-\alpha$ quantile of the posterior distributions of $\lambda$ calculated with the user-provided column of $\rho$ values and with the fitted $\rho$ values is taken as a rough estimate of the relative bias.

\subsubsection{Track Past Mortality}

Under the "track past mortality" option, the estimated annual and cumulative mortality is estimated for each year of past search data (as entered in the Past monitoring and operations data table). The user enters the number of years in the project and a mortality threshold (T), which may be a longterm take limit or some other significant mortality level. The number of years in the project and $\mathrm{T}$ are not used in the calculations to track past mortality but are used to define bounds on a graph of results. Results are shown both in graphical and text format.

In the tracking graph (fig. 13), posterior distributions of the cumulative mortality $(M)$ through the monitored years are shown as color bars, and point and interval estimates of the overall detection probability for the entire period and of the baseline fatality rate. 


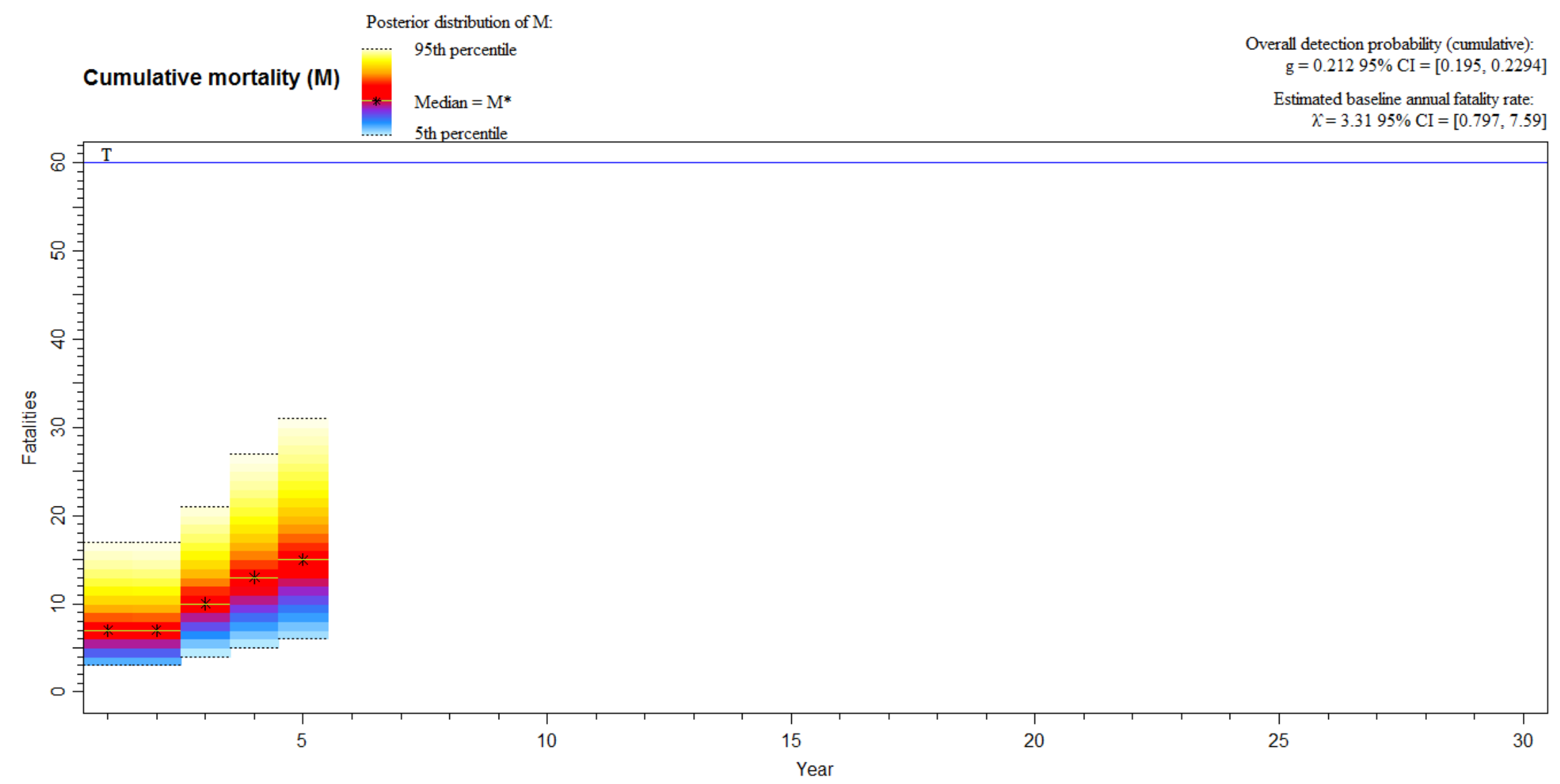

Figure 13. Graph generated by Evidence of Absence software showing estimated cumulative mortality through the monitored years. 
Posterior distributions of the annual fatality rates $(\lambda)$ for every year monitored are given in a separate graph (fig. 14). The estimates of annual rates are based solely on the search data for the given year and do not take into consideration assumed relative rates, $\rho$.

Results in text format (table 7) include summary statistics for the total mortality through the monitored years, including $M^{*}$ and point and interval estimates for overall detection rate $(g)$ and baseline fatality rate $(\lambda)$. In addition, summary statistics for estimates of cumulative and annual mortality are given for each year. Finally, results of the tests for validity of the assumed weights $(\rho)$ and for potential bias are given because mortality estimates may not be accurate if $\rho_{i}$ 's are significantly misspecified. These tests are described in more detail in section F.3.

\section{Annual posterior median $\lambda$ with IQR and $95 \% \mathrm{Cl}$}

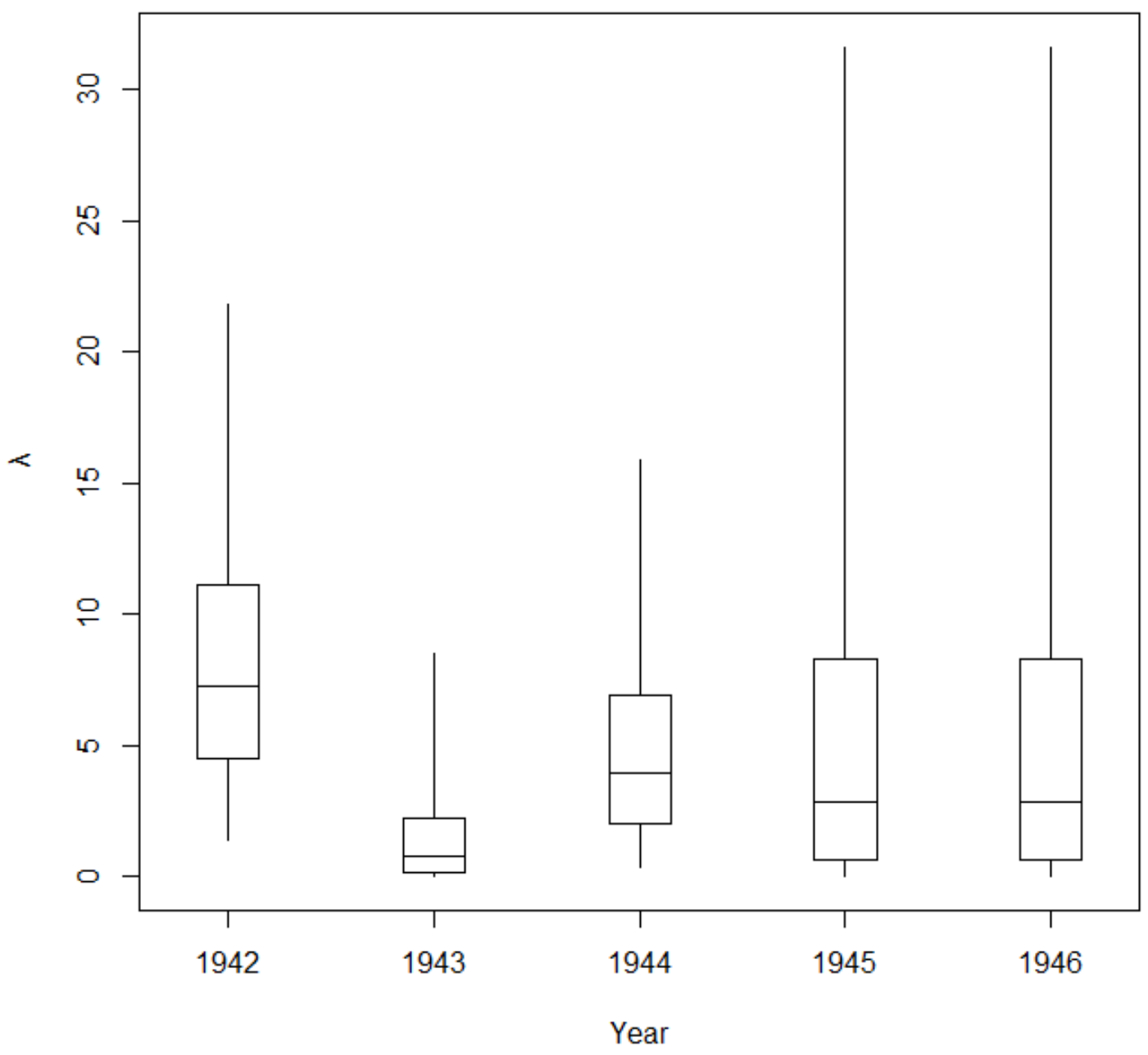

Figure 14. Box and whisker plots generated by Evidence of Absence software showing posterior distributions of $\lambda$ for each year that monitoring data were available. 
Table 7. Results table generated by Evidence of Absence software showing cumulative and annual mortality estimate.

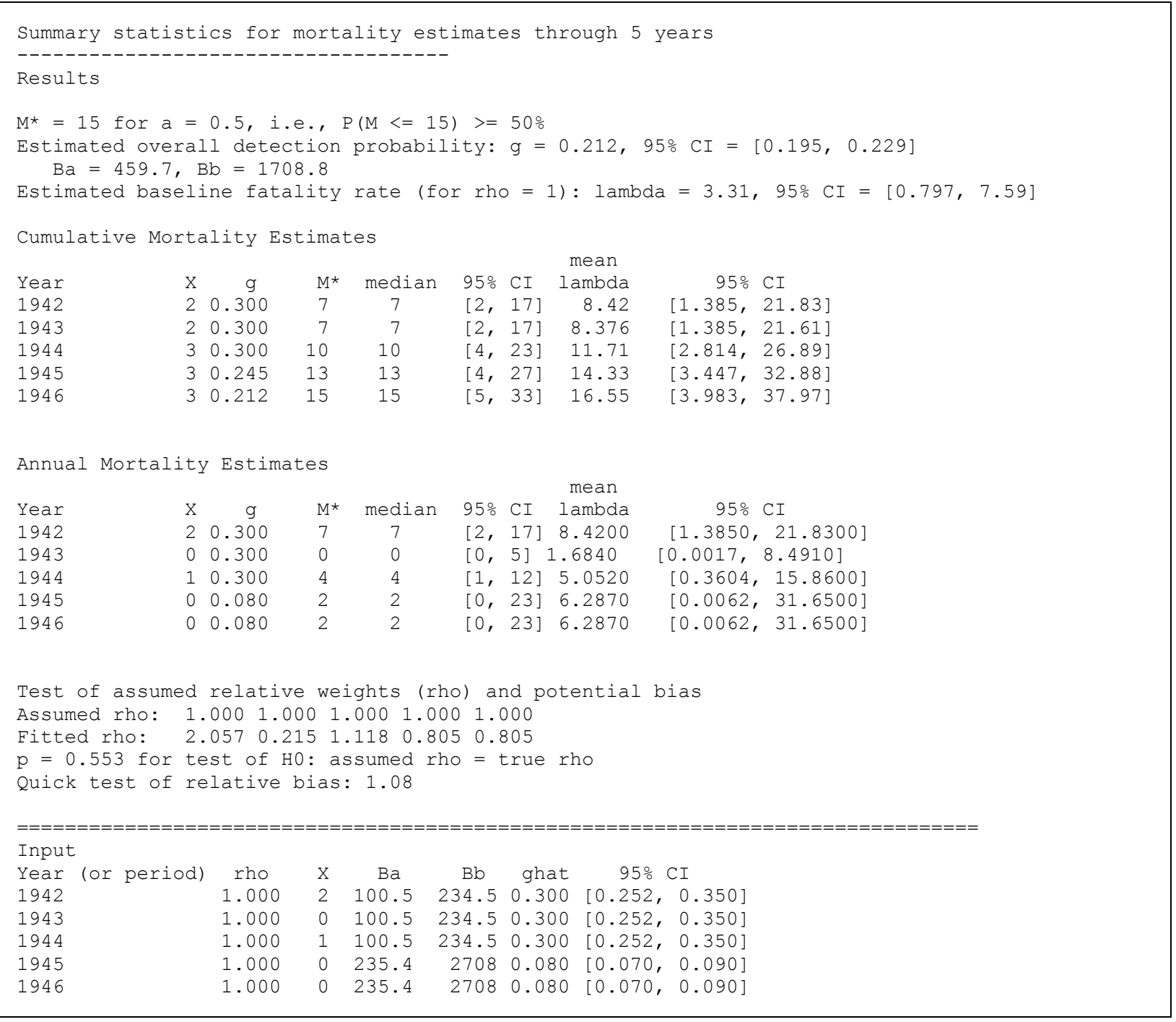




\subsubsection{Projection of Future Mortality and Estimates}

For the projection analysis, the user enters past search data in the Past monitoring and operations data table and anticipated future monitoring parameters and relative fatality rates. In turn, EoA simulates future mortality and search processes. Then, the EoA model is used to make projected fatality estimates from the simulated future search results. Output includes summary statistics for projected mortality $(M)$, projected mortality estimates $\left(M^{*}\right)$, and projected mortality threshold exceedance patterns.

There are three options for entering future monitoring and operations parameters. Under option $g$ and $\rho$ unchanged from most recent year, parameters that were used in the most recent year of past monitoring are applied to all future years as well. Under option $g$ and $\rho$ constant, different from most recent year, the user enters parameter values for the first projection year, which remain constant for all projection years. Under option $g$ and $\rho$ vary among future years, the user may edit the parameters values in the Future monitoring and operations parameters table manually. Values may be typed into the table or pasted from the clipboard. To navigate within the table, use the arrow keys. To append or delete rows, edit the Total years in project. A parameter conversion calculator is available via the Help menu to convert from point and interval estimates of $g$ to approximately equivalent beta distribution $B a$ and $B b$ parameters (and vice versa). The conversion from $\hat{g}$ with $95 \%$ CI to $B a$ and $B b$ is not exact because there is no guarantee that a given combination of $\hat{g}$ with $95 \%$ CI will have a beta distribution to match (appendix J).

Based on the past data, the software estimates the baseline mortality rate ( $\lambda$ for years with $\rho=$ 1) and then generates simulated fatalities for future years at a rate of $\lambda \rho_{i}$ for year each year $i$, where $\rho_{i}$ is the user-defined anticipated relative weight for year $i$.

For each future year, the mean, median, IQR, and $80 \%$ and $90 \%$ PI's of the posterior predictive distributions of both the cumulative mortality and corresponding mortality estimates (appendix F, section F.2) are given in graphical and text format.

Featured in the Cumulative mortality plot (fig. 15) for each year until the end of the project are (1) box-and-whisker plots of projected actual mortality, $M$, and (2) gray color bars showing the projected estimated mortality, $M^{*}$, with light gray lines indicating the median. The box and whisker plots show projected numbers of actual fatalities and are of interest to conservation. However, because the true number of fatalities will never be known with certainty, future management decisions will be based on estimated mortalities, and the gray bars give an indication of what the available mortality information will look like to managers in future years.

With the default data set, in year 20 the median projected mortality $(M)$ is equal to the mortality threshold $\mathrm{T}$, indicating that if the mortality rate does not change, there is a $50 \%$ chance that the cumulative mortality will exceed T in year 20 (fig. 15). However, the median projected estimate of mortality $\left(M^{*}\right)$ is clearly less than T in year 20 (light gray line) but greater than $\mathrm{T}$ in year 21 , indicating that there is somewhat less than 50-50 odds that the mortality estimate in year 20 will exceed $\mathrm{T}$ and slightly greater than 50-50 odds that it will exceed $\mathrm{T}$ in year 21 .

Results also are given in tabular format. The output file has been split into two parts for discussion, with summary statistics for future projections shown in table 8 and summary statistics for past estimates shown in table 9. 


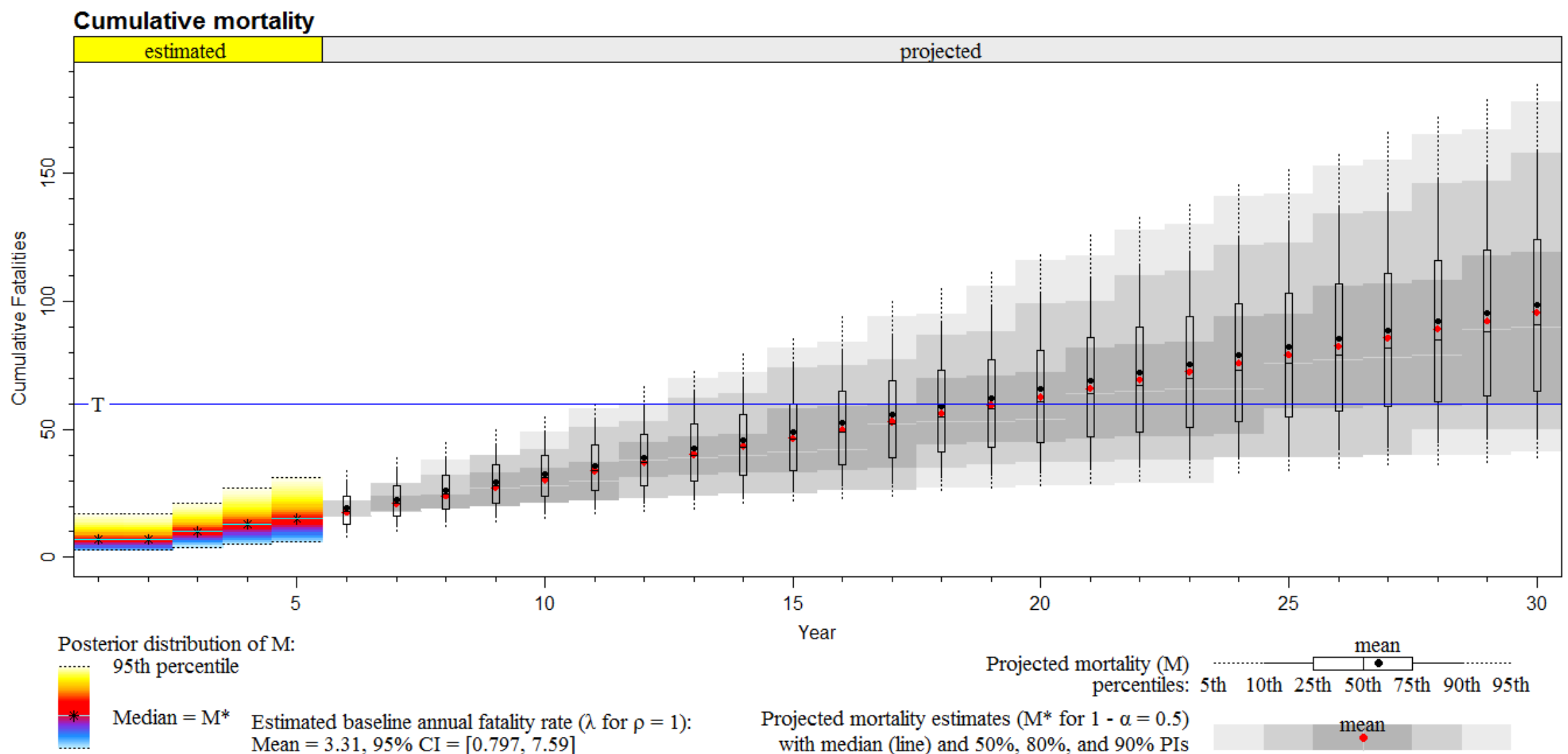

Figure 15. Box and whisker plots generated by Evidence of Absence software showing estimates of past mortality and projections of future mortality and mortality estimates, $\alpha=0.5$. 
Table 8. Results table generated by Evidence of Absence software showing summary statistics for future mortality projections, $1-\alpha=0.5$ (part A).

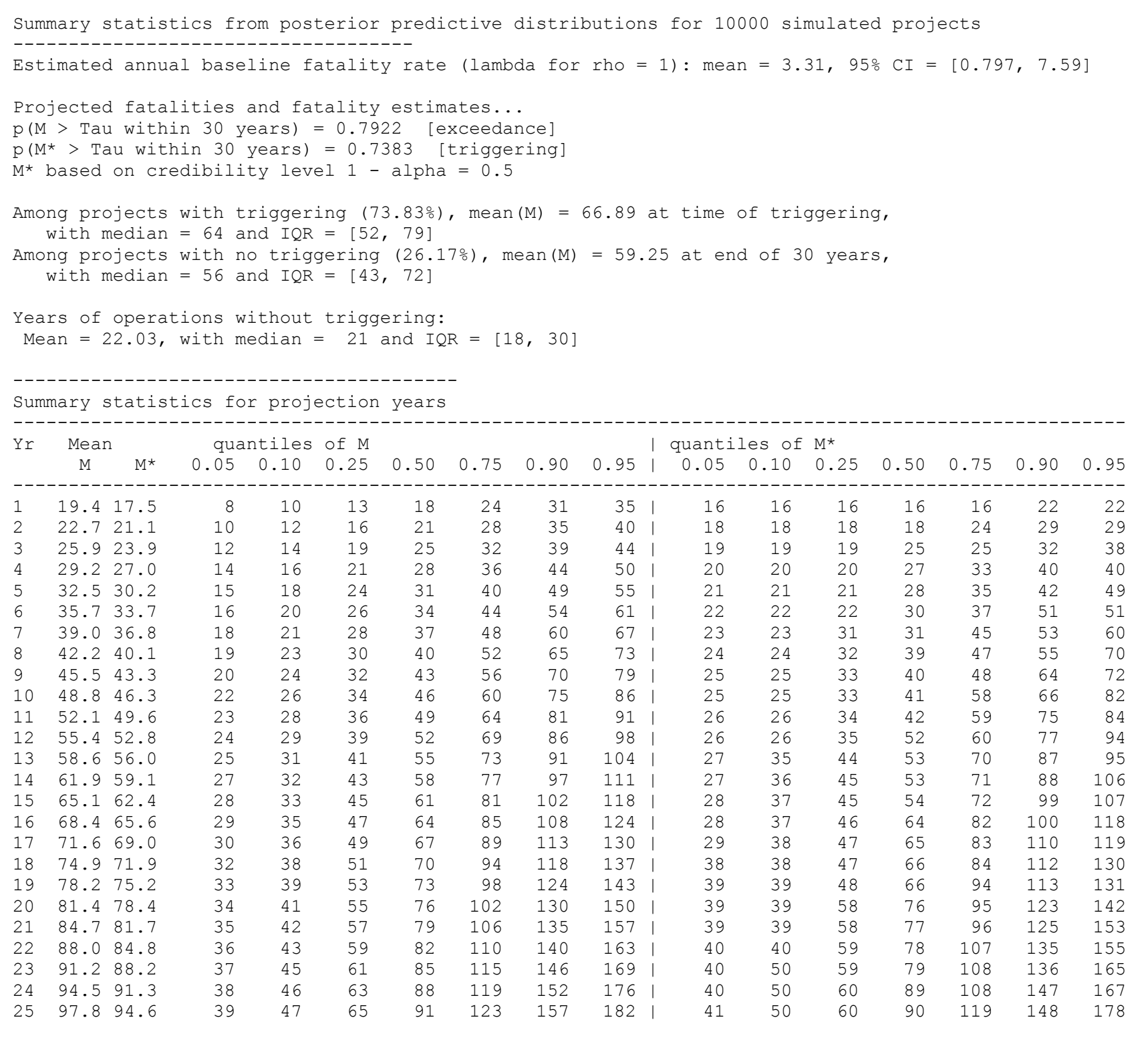


Table 9. Results table generated by Evidence of Absence software showing summary statistics for past mortality estimates, $1-\alpha=0.5$ (part B).

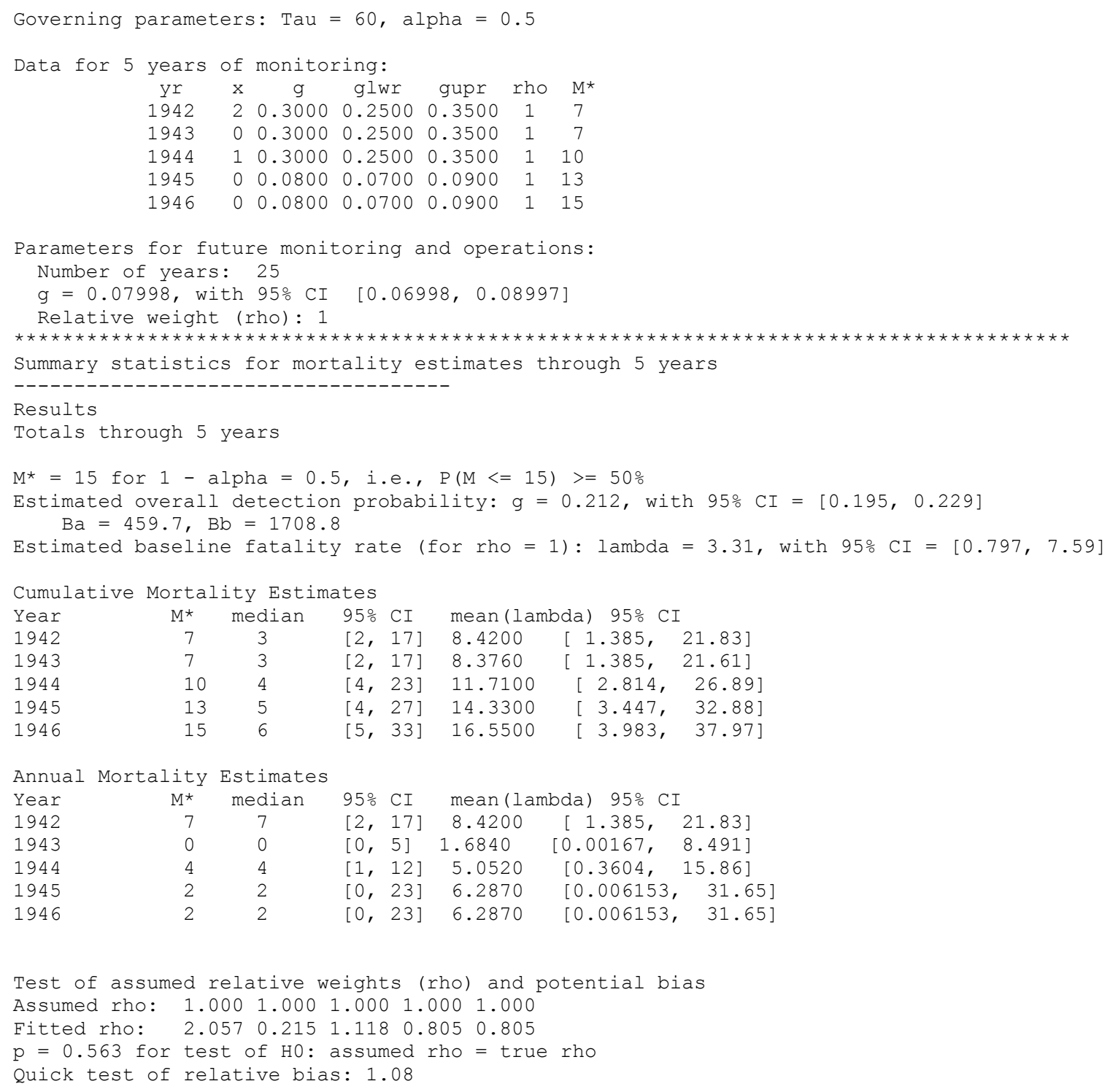


Projections (table 8) are based on the estimated annual baseline fatality rate $(\lambda)$, which is the expected number of fatalities in a year with relative weight $\rho=1$. In general, the expected fatality rate in a year with relative weight $\rho$ will be $\rho \lambda$. The baseline fatality rate is not known precisely and must be estimated from past monitoring data. The uncertainty is characterized in the posterior distribution of $\lambda$, which is summarized in the results table with the mean and $95 \% \mathrm{CI}$, or mean $(\lambda)=3.31$ and $95 \% \mathrm{CI}=$ $[0.797,7.59]$ for the default data set. With the default parameter set, the actual number of fatalities exceeded the threshold in about $79 \%$ of the 10,000 simulated projects, $P(M>\mathrm{T})=0.7922$. However, the estimated number of fatalities exceeded the threshold slightly less often, $P\left(M^{*}>\mathrm{T}\right)=0.7383$. The average number of fatalities at the time of triggering - that is, the time that the estimated number of fatalities first exceeded T-was 66.89, which is slightly greater than $\mathrm{T}$ and reflects the design of the trigger to signal when the threshold is exceeded. The mean number of fatalities in projects with no triggering - that is, $M^{*} \leq \mathrm{T}$ at the end of the permit term-was 59.35, which is slightly less than T.

More extensive summary statistics for projection years also are provided (table 8), including the means and $0.05,0.10,0.25,0.50,0.75,0.90$, and 0.95 quantiles for both $M$ and $M^{*}$ for each projection year. Results for the default data set show that the mean $M^{*}$ tracked the mean $M$ fairly closely but was slightly smaller each year. A slight gap between average projected cumulative $M^{*}$ and $M$ is common because cumulative $M$ increases in regular steps from year to year while $M^{*}$ follows a more broken pattern with periods with almost constant $M^{*}$ followed by years when $M^{*}$ increases in great leaps, as can be seen (fig. 15) by comparing the median bars of $M$ (center lines in the box-and-whiskerplots) with the median bars of $M^{*}$ (light gray horizontal lines). A rough explanation is that while $M$ increases by (approximately) $\lambda$ each year, $M^{*}$ remains relatively constant when no carcasses are found but increases by (approximately) $1 / g$ whenever another carcass is found. After each leap, median $M^{*}$ is closely aligned with median $M$, and the medians gradually diverge until another leap in $M^{*}$ (by $1 / g$ ) brings them back into alignment. By contrast, the mean of $M^{*}$ (red dots) increases in regular steps and generally lies close to the median $M$.

Another noteworthy pattern in comparing the distributions of $M$ with the distributions of $M^{*}$ through time is that in the early projection years, $M$ is substantially more variable than $M^{*}$ but the difference becomes gradually less pronounced through time (table 8, fig. 15). For example, in the first projected year (or 6th year of the project), the $90 \%$ PI of $M$ is $[8,35]$, while the $90 \%$ PI of $M^{*}$ is $[16$, 22]. The PI for $M^{*}$ is relatively narrow because it is derived from the cumulative carcass count, which will change very little with one additional year of monitoring. There is relatively little uncertainty about what the estimate of cumulative mortality will be after an additional year of monitoring. If no additional carcasses are found, $M^{*}$ for years $1-6$ will be virtually unchanged from $M^{*}$ for years $1-5$. If one carcass is found in year $6, M^{*}$ for years $1-6$ will increase by approximately $1 / g$ compared to $M^{*}$ for years $1-5$.

The past data and future assumptions that the projections are derived from are reproduced in the results table (table 9), below the projections themselves and above the summary statistics for mortality estimates for past years. The summary statistics for past years mirror those in the tracking graph (fig. 15) and table (table 9). 
A final graph for the mortality projections tool shows the posterior predictive probabilities of exceedance (that is, actual mortality exceeding threshold, $P(M>\mathrm{T})$ ) and of triggering (that is, estimated mortality exceeding the threshold, $P\left(M^{*}>\mathrm{T}\right)$ ). For example, with the default scenario (fig. 16), in any given year the probability that cumulative mortality exceeded threshold (black line) was typically slightly greater than the probability that the estimated mortality exceeded the threshold (red line).

Basing $M^{*}$ on $\alpha=0.2$ (fig. 17) instead of 0.5 (fig. 15) has no effect on projected mortality ( $\left.M\right)$, but projected mortality estimates $\left(M^{*}\right)$ are greater. Triggering occurs earlier and typically precedes actual exceedance $(M>\mathrm{T})$ by several years. When $\alpha=0.2$ with the default data set, mean $M^{*}$ first exceeds $\mathrm{T}$ in year 15 instead of year 20 as it did when $\alpha=0.5$.

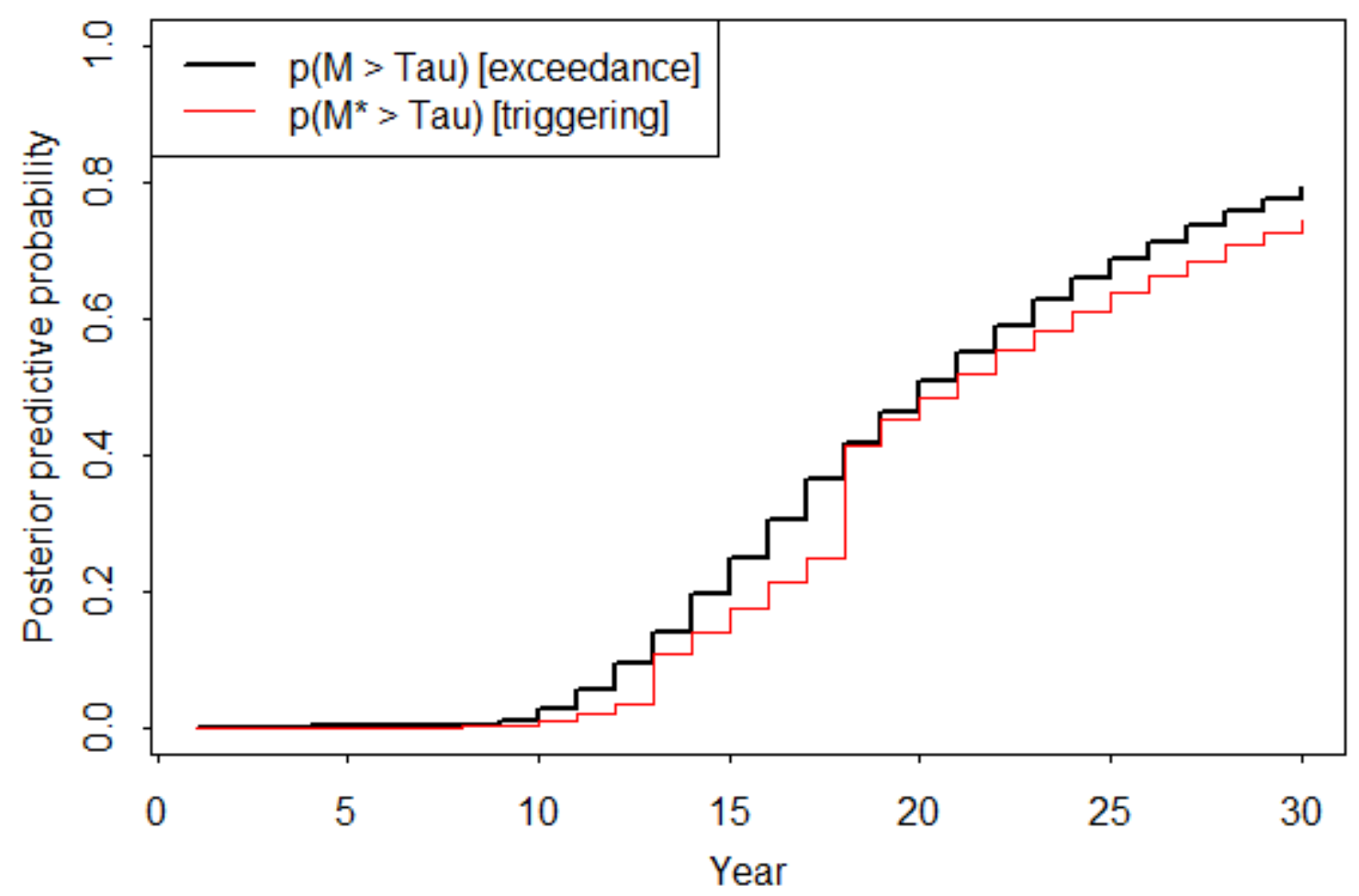

Figure 16. Graph generated by Evidence of Absence software showing probabilities of exceedance and triggering for each year. 


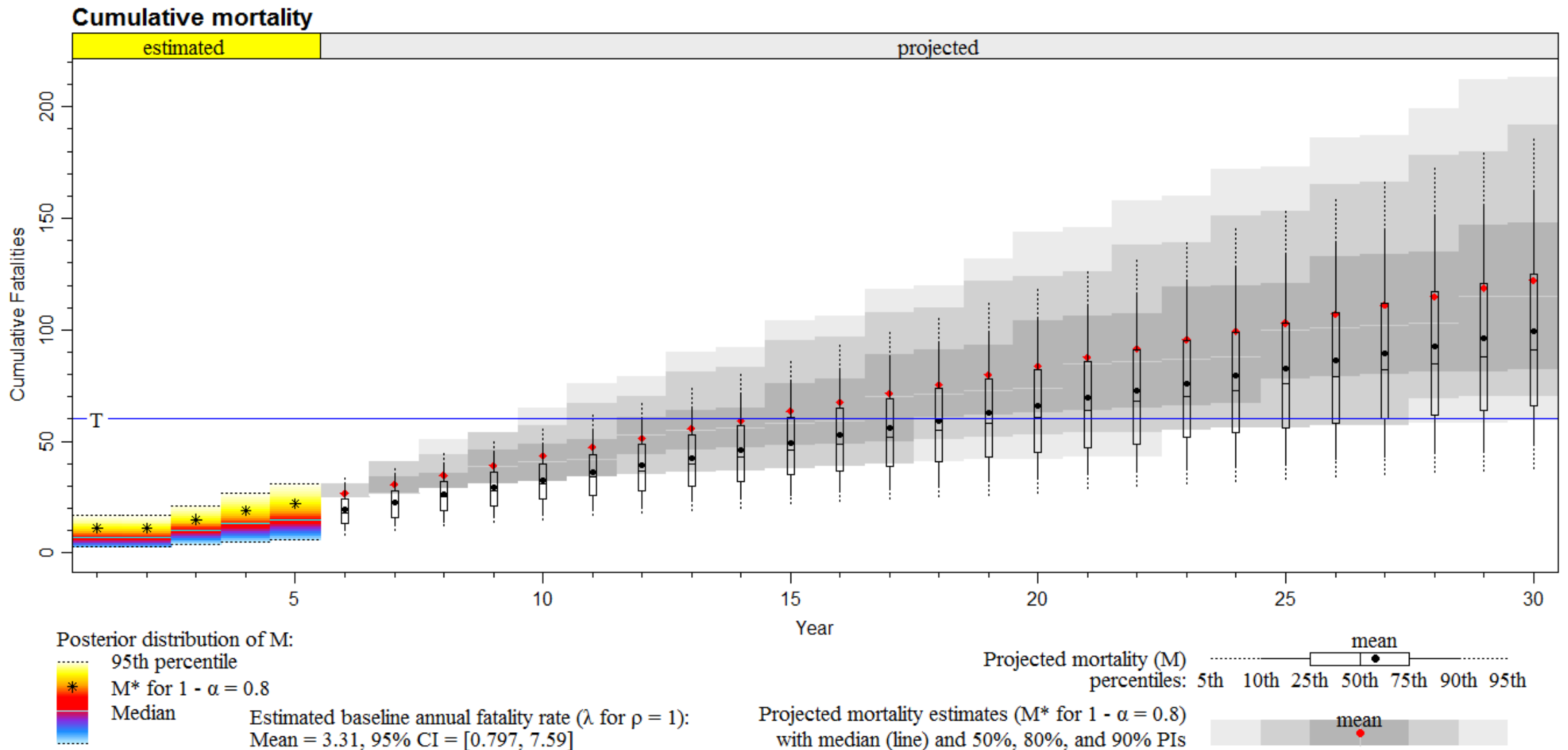

Figure 17. Box and whisker plots generated by Evidence of Absence software showing estimates of past mortality and projections of future mortality and mortality estimates, $1-\alpha=0.8$. 
Although the relative weight, $\rho$, usually has little effect on estimates of past mortality, it strongly affects fatality projections. The software's default option for the projection years is to set $\rho$ at whatever it was in the final year of monitoring, with the interpretation that future mortality rates will not change from the most recent year monitored. However, the user may override the default to reflect anticipated changes in operations or to explore "what if..."scenarios. For example, setting $\rho$ equal to 3 in the default data set beginning with the 7 th projection year (fig. 18) indicates that the annual mortality rate from the first 5 years of monitoring remains the same for the next 6 years but then triples in year 12 . The effect can be seen as a clear trend break in year 12 (fig. 19) for both $M$ and $M^{*}$. Mean estimated mortality first exceeds $T$ in year 14, and actual number of fatalities exceeds 200 by the end of the 30 years, in contrast to the mean of less than 100 when $\rho$ is held constant at 1 .

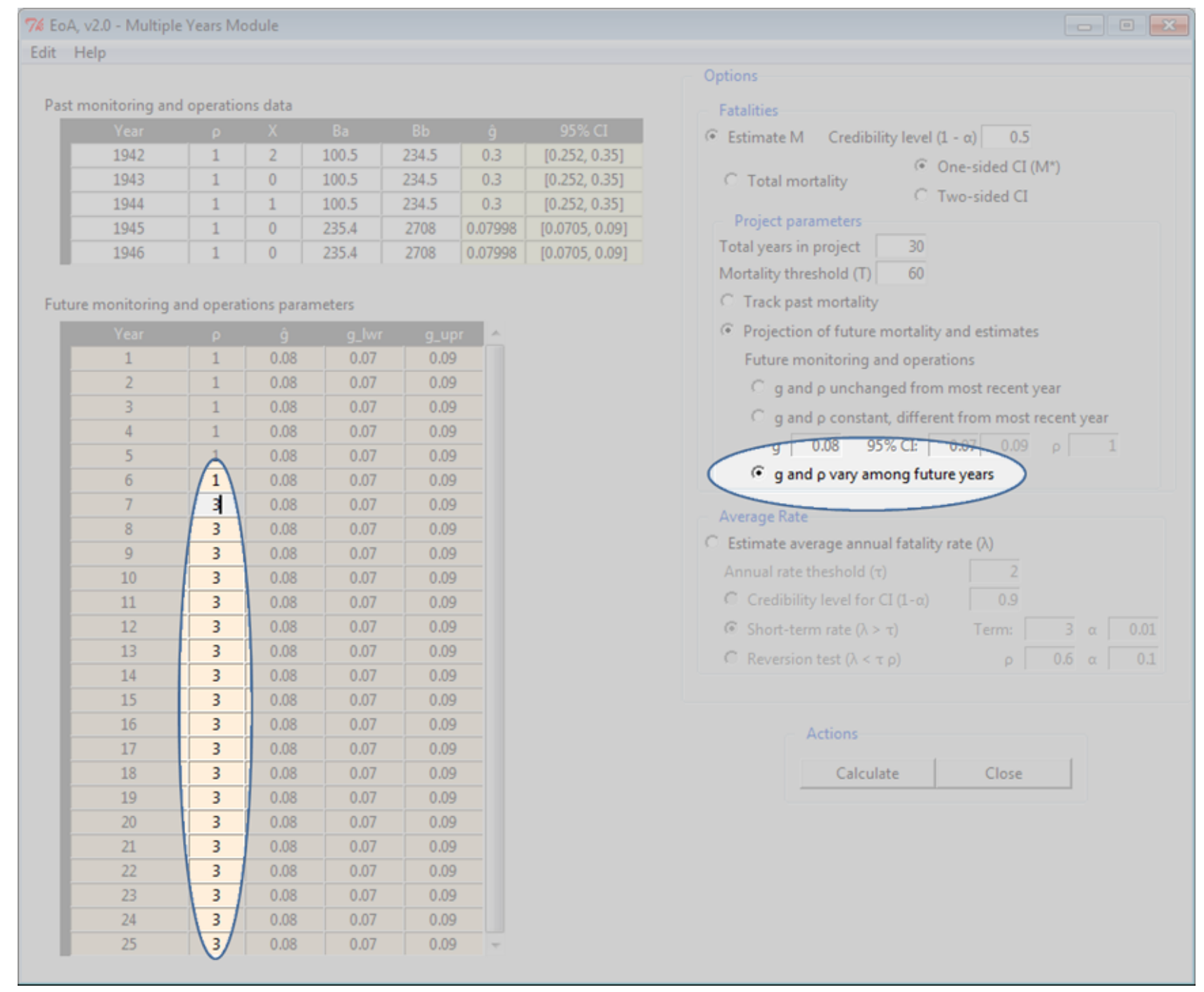

Figure 18. Screen capture of the Multiple Years Module, accounting for anticipated changes in operations in future years. In projection year 7 , mortality rate triples due to expansion of the site. 


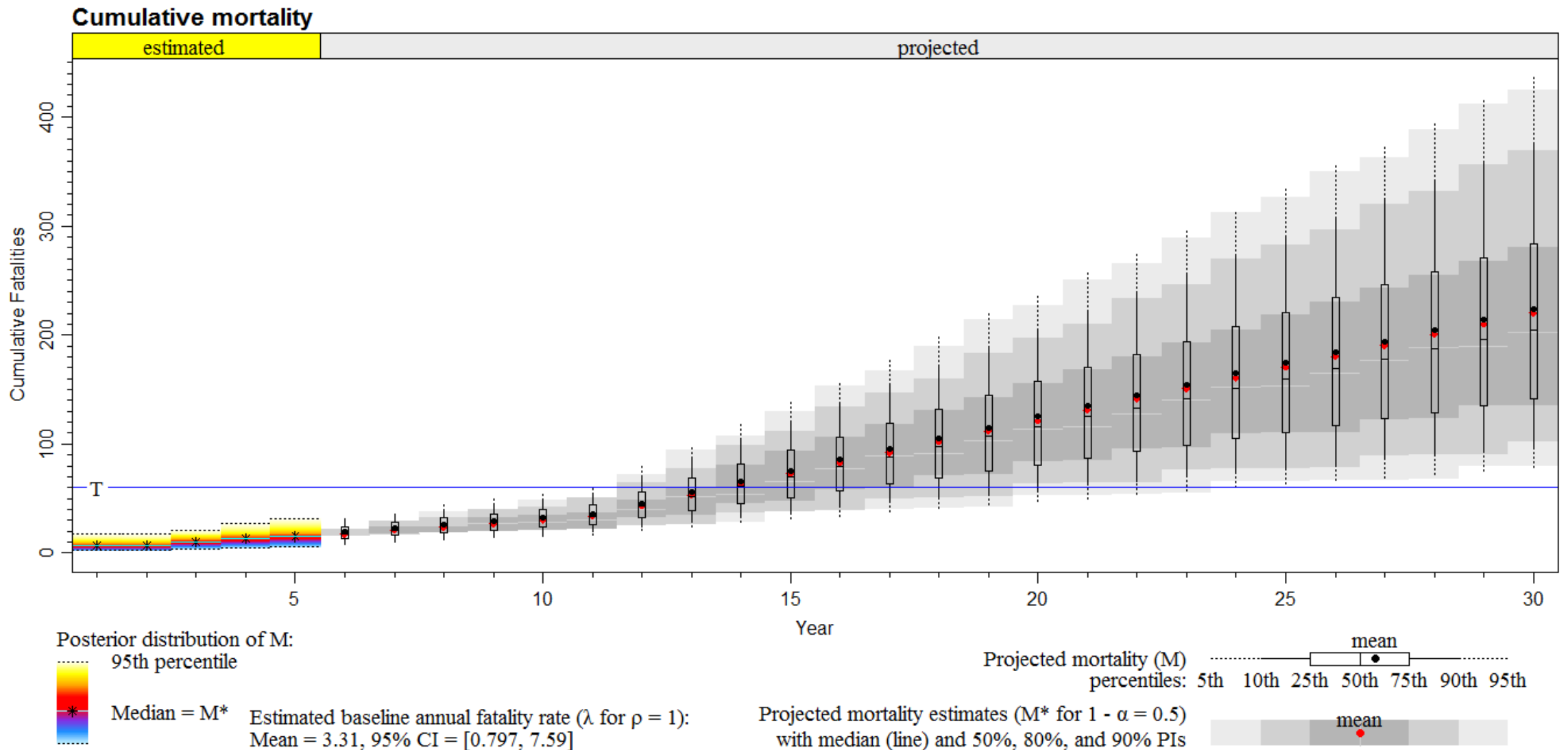

Figure 19. Box and whisker plots generated by Evidence of Absence software showing projected mortality with anticipated tripling of fatality rate $(\rho=3)$ beginning in year 12 (or projection year 7). 


\subsubsection{Estimate Average Annual Fatality Rate $(\lambda)$}

Carcasses are generated by a random process at an average rate of $\lambda$ per year. If in one particular year, we know with certainty that there were exactly $M$ fatalities, there is still uncertainty about $\lambda$ because the number killed would vary from year to year due to random variation. However, $\hat{g}$ and $X$ provide information about the average annual rate that is analyzed and summarized using the Average Rate tools (fig. 20) in the Multiple Years Module. Options include calculating a CI, performing a shortterm test on the rate $(\lambda>\tau)$, or performing a reversion test $(\lambda<\tau \rho)$.

In the option to calculate a $\mathrm{CI}$, the user enters the desired credibility level, $1-\alpha$. Output includes a $100(1-\alpha) \% \mathrm{CI}$ for the average annual mortality rate $\lambda$ for the years monitored and a $95 \%$ $\mathrm{CI}$ for the overall detection probability for carcasses arriving in the years monitored. A rough interpretation of the $\mathrm{CI}$ for $\lambda$ is that there is $100(1-\alpha) \%$ probability that $\lambda$ is in the interval. The bounds of the CI $\left(\lambda_{\text {lwr }}\right.$ and $\left.\lambda_{\text {upr }}\right)$ are defined to be the points where $P\left(\lambda<\lambda_{\text {lwr }}\right)=P\left(\lambda>\lambda_{\text {upr }}\right)=\alpha / 2$ according to the posterior distribution of $\lambda$ derived from the pooled estimate of overall detection probability and pooled carcass counts ( section G.2).

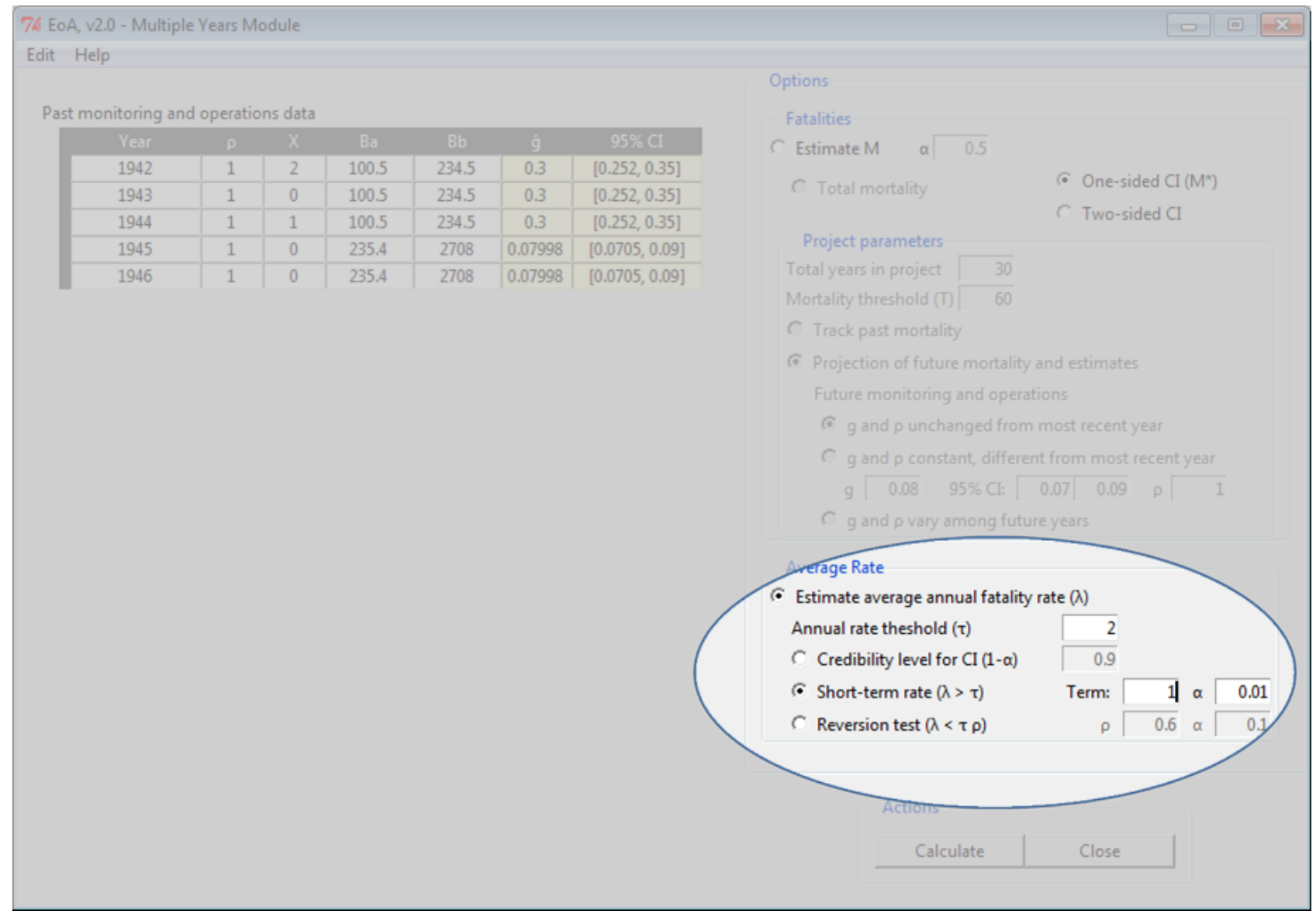

Figure 20. Screen capture of Multiple Years Module, estimating average fatality rate $(\lambda)$ in past years. 
The short-term test estimates the average annual fatality rate over the past few years. The shortterm test can be used to determine if past monitoring data signal a potentially unsustainable mortality rate that would lead to exceedance of the long-term limit before the end of the permit term. The user inputs the annual rate threshold $(\tau)$, the number of years over which to calculate the rate (Term), and the sensitivity of the test $(\alpha)$. For example, in the default data set (fig. 11), $\tau$ is set equal to 2 to reflect a management or regulatory concern that an annual rate of $\lambda>2$ would likely lead to exceedance of the long-term fatality limit of $\mathrm{T}=60$ over the permit term of 30 years. The rate is estimated from the most recent $n$ years, as specified by the user. To estimate the previous year's rate, enter a value of 1 for the Term. The 95\% CI for the rate is $[0.00615,31.6]$ (table 10), which appears to be a very wide range. The problem is that the overall detection probability was only 0.08 , which implies that we would expect to miss $92 \%$ of the carcasses, so the data do not support a precise estimate of the rate. The test of whether there is sufficient evidence that the short-term rate exceeds $\tau$ is based on the probability that $\lambda>\tau$ according to the posterior distribution of $\lambda$. If $P(\lambda>\tau)>1-\alpha$, the inference is that $\lambda$ is significantly greater than $\tau$. The user-defined value of $\alpha$ is a statement of the strength of the evidence required before concluding $\lambda>\tau$. Smaller $\alpha$ 's mean more stringent demands for evidence. Typically, a small value of $\alpha$ (like $\alpha=0.01$ ) is used for the short-term test. If the test is conducted year after after, larger values of $\alpha$ can result in frequent false alarms where the inferred "exceedance" is due simply to random chance rather than actual exceedance.

The short-term test provides valuable information about whether a given rate has been exceeded and can serve as an early warning of potentially excessive take. However, some caveats about running the short-term rate test with a single-year term are: (1) the amount of information in a single year of monitoring tends to be scarce, so CIs tend to be wide, and (2) there may be some year-to-year fluctuation in $\lambda$, so a single year's data might not provide a reliable estimate of the annual rate over the long term. Increasing the term to 2 or 3 or more years can ameliorate both these potential problems. For example, increasing the term from 1 year to 2 years in the previous example (fig. 20) cuts the CI width in half, from $[0.00615,31.6]$ to $[0.00307,15.8]$ because the second consecutive year with $\mathrm{X}=0$ gives stronger evidence of a smaller rate.

Table 10. Results generated by Evidence of Absence software showing short-term trigger test results.

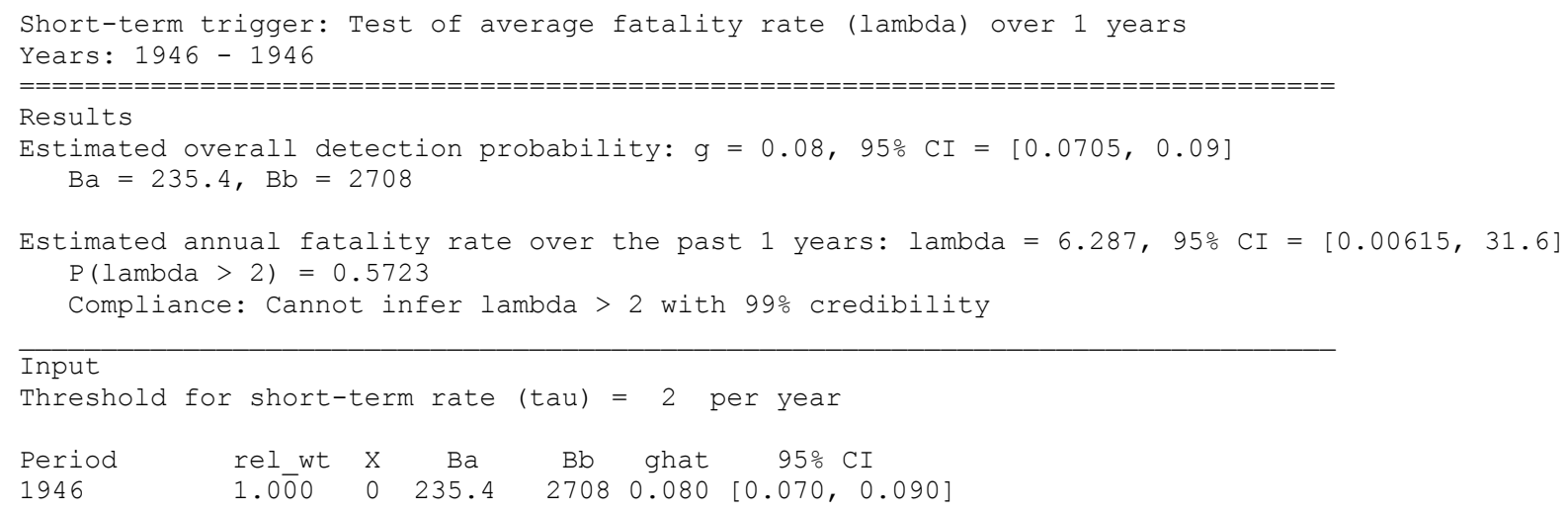


The reversion test is a specialized test to determine if the fatality rate in the monitored years is significantly lower than a given level that would cause concern or whether rates are low enough to warrant the relaxing of operational constraints imposed for species conservation. For example, suppose that a mortality rate of $>\tau$ would raise legitimate conservation concerns, and a wind power facility is required to monitor intensively to give assurance that $\tau$ is not exceeded. The intensive monitoring showed that the mortality rate during those years was significantly smaller than $\tau$, and less stringent monitoring requirements were implemented as a result. For another example, suppose that a new wind power facility seeks to ensure that annual mortality rates are below $\tau$ and employs prophylactic minimization measures such as curtailment or deterrents to reduce mortality rates by a factor of $\rho$ compared to what would otherwise be expected. If the fatality rate under the minimization regime is $\lambda$, then it will be $\lambda / \rho$ if the minimization measures are discontinued. Thus, if $\lambda$ is demonstratively less than $\rho \tau$, then the mortality rate after discontinuing the minimization measures would be expected to be less than $\tau$. For example, with the default data set (fig. 11), the reversion test determines whether $\lambda<\rho \tau=$ $0.6 \cdot 2=1.2$. The results show that the average annual fatality rate was 3.31 , which is not significantly smaller than 1.2, and the conclusion is "No reversion: Cannot infer $\lambda<\rho \tau=1.2$ with $90 \%$ credibility" (table 11). Smaller values of $\alpha$ mean stronger evidence is required before allowing reversion.

Table 11. Reversion trigger test results.

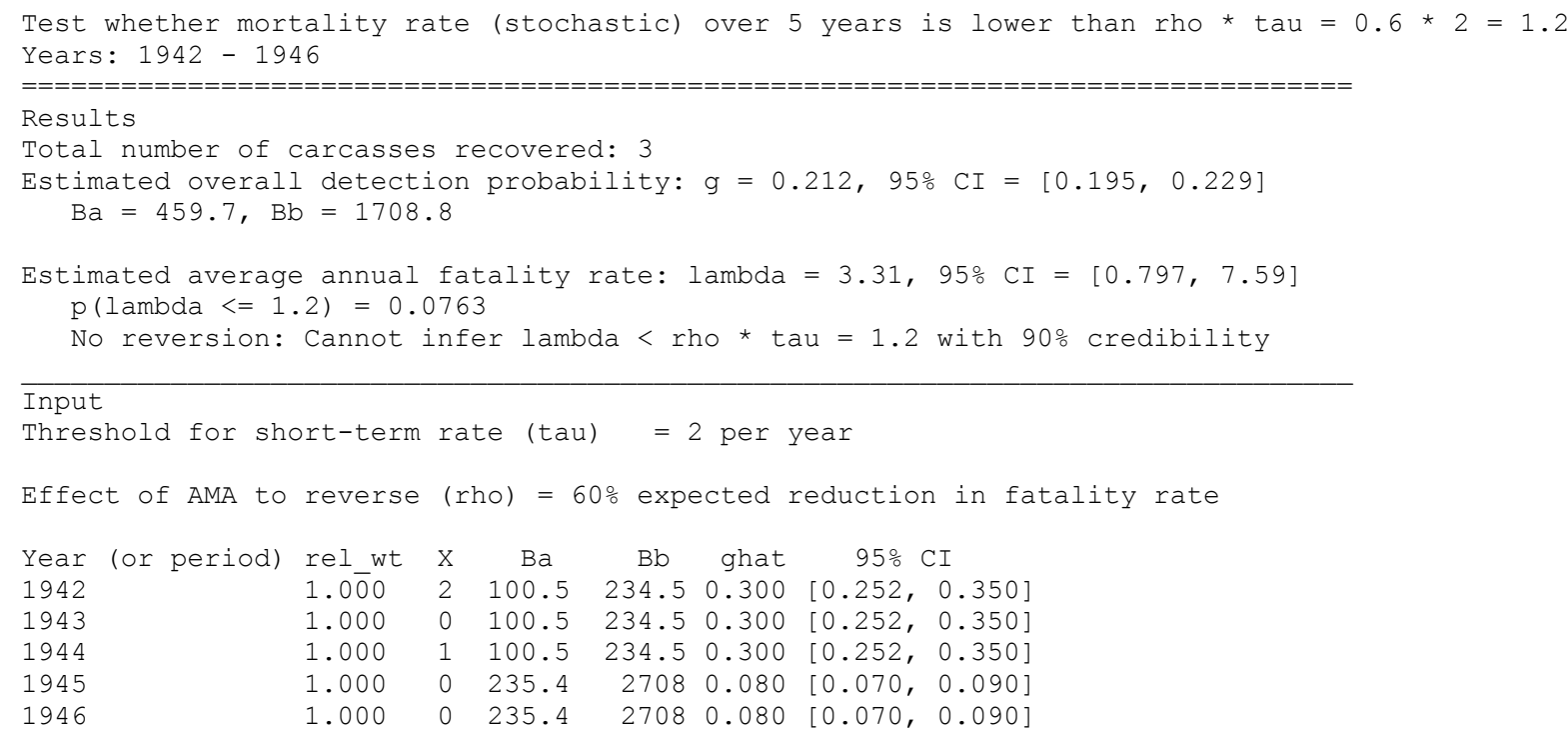




\section{Design Tradeoffs}

The Design Tradeoffs module (fig. 21) provides tools for optimizing study design to achieve a stated design objective, which may be to determine what management parameter values are required to attain a target detection probability $(g)$ or a target credibility level $(1-\alpha)$ for ruling out the possibility that mortality exceeds a given threshold for a hypothetical number of carcasses found. The user inputs ranges of values for proposed searcher efficiency, search coverage, and search schedule, and software displays graphs of the resulting detection probabilities or credibility levels for the given combinations of monitoring parameters.

Data may be entered manually or by importing a .rds file with parameter sets previously saved by selecting Save to file (.rds) from the Edit menu. Upon performing calculations, the active parameter set is saved for reloading the next time the module is opened. If desired, the default parameter set may be reloaded by selecting Restore defaults from the Edit menu.

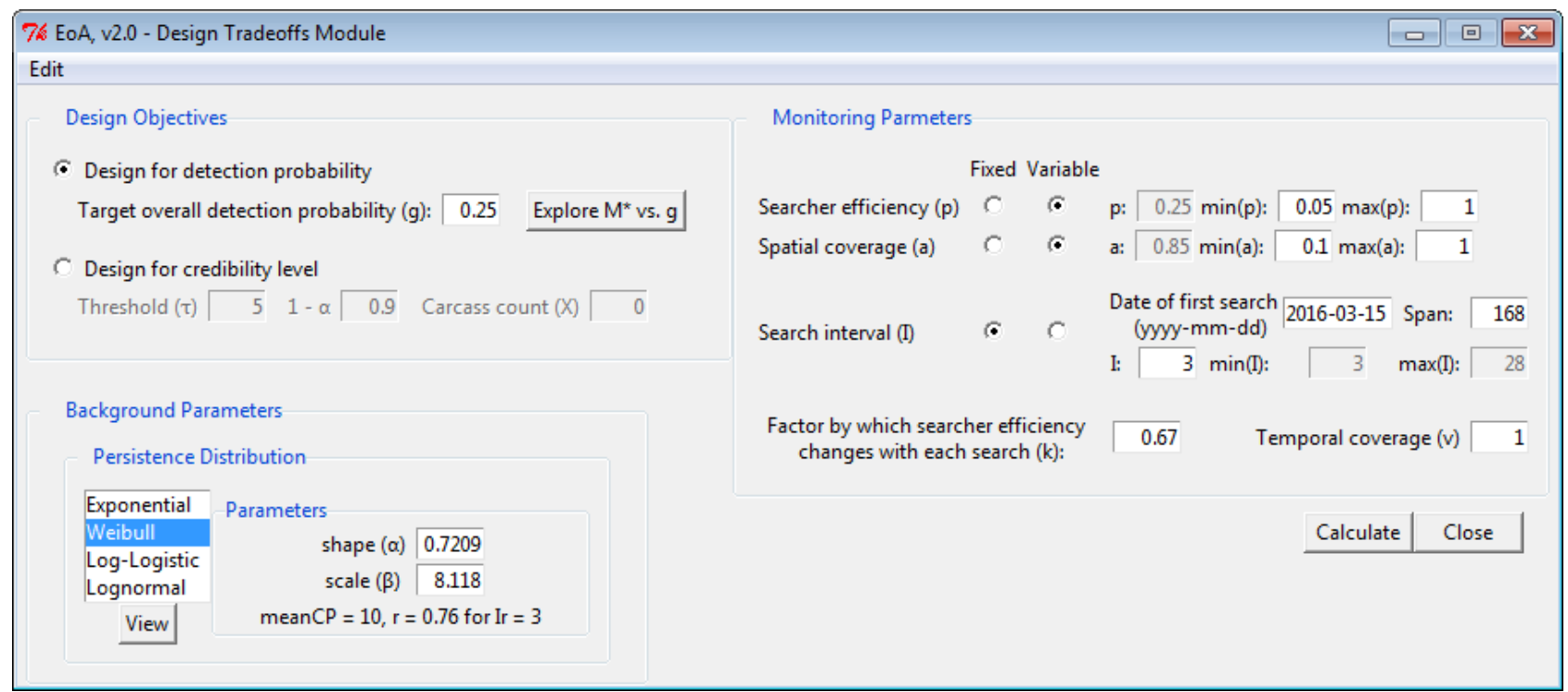

Figure 21. Screen capture of Design Tradeoffs Module (inputs). 


\subsection{Design Objectives}

The user selects one of two objectives: to optimize the study design to achieve a target overall Detection Probability $(\boldsymbol{g})$ or to achieve a target Credibility Level $(\mathbf{1}-\boldsymbol{\alpha})$ in providing assurance that a specified threshold has not been exceeded when a hypothetical number of carcasses has been found. A tool is provided for exploring the relationship between $M^{*}$ and $g$ (fig. 22) to help the user determine a desired target detection probability level.

Alternatively, the user can input parameters to design a monitoring program to provide 100(1 $\alpha) \%$ credibility that a specified Threshold $(\boldsymbol{\tau})$ has not been exceed, given a hypothetical Carcass count (X).

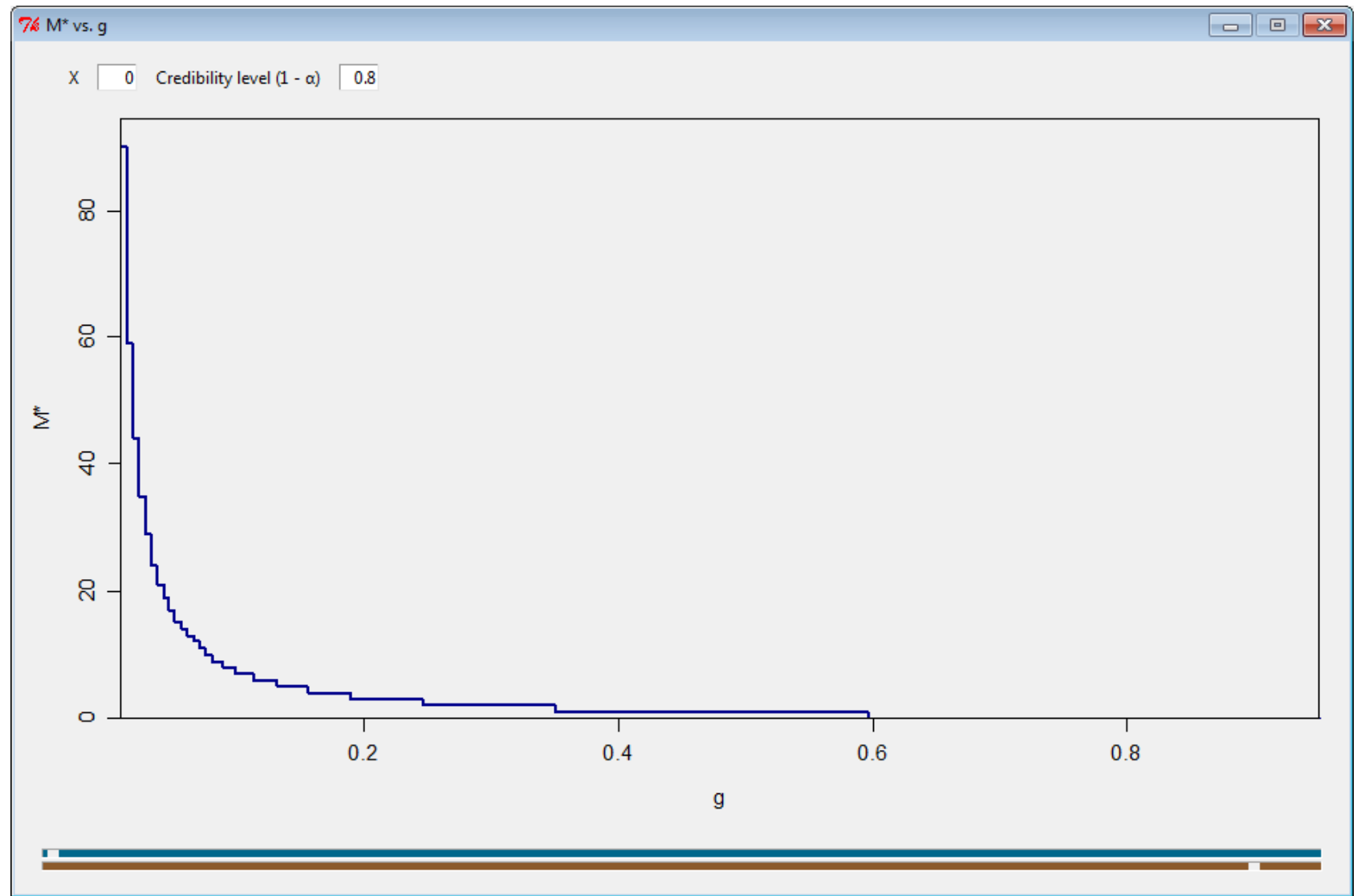

Figure 22. Screen capture of $M^{*}$ vs $g$ option. Sliders control the limits of the $x$-axis, allowing the user to zoom in on a desired range of $g$. 


\subsection{Monitoring Parameters}

Four factors generally determine the overall detection probability: searcher efficiency, search coverage, search schedule, and persistence pattern. The first three, listed under Monitoring

Parameters, are factors over which we have most control. For example, searcher efficiency can be improved by clearing vegetation within the searched area or using well-trained dogs (Arnett, 2006; Paula and others , 2011; Mathew and others, 2013) to search in thick vegetation; spatial coverage can be improved by searching a higher proportion of turbines at a site, increasing the search radius, or searching areas with highest density of carcasses; and reducing the search interval leaves less time for carcasses to be removed between searches, thereby increasing overall detection rates.

The user has the option to enter fixed values or ranges for searcher efficiency $(p)$, spatial coverage $(a)$, and search interval $(I)$. In addition, the user enters the date of the first search, the span of the monitored period (that is, the number of days between the first and last searches), the factor by which searcher efficiency changes with each search $(k$, section 2.1.2), and the temporal coverage $(v$, section 2.1.1).

\subsection{Background Parameters}

The Persistence Distribution often strongly influences the detection probability and is listed under Background Parameters. Generally, the persistence patterns are determined by the scavenging population at a site and are harder to change than the monitoring parameters are. However, there may be some opportunity to increase persistence times by removing or deterring scavengers or by constructing exclusion cages around searched areas. User selects the appropriate persistence distribution and enters the governing parameters of the distribution (section 2.1.3). The View button produces a graph representing the persistence pattern of the input distribution (fig. 23).

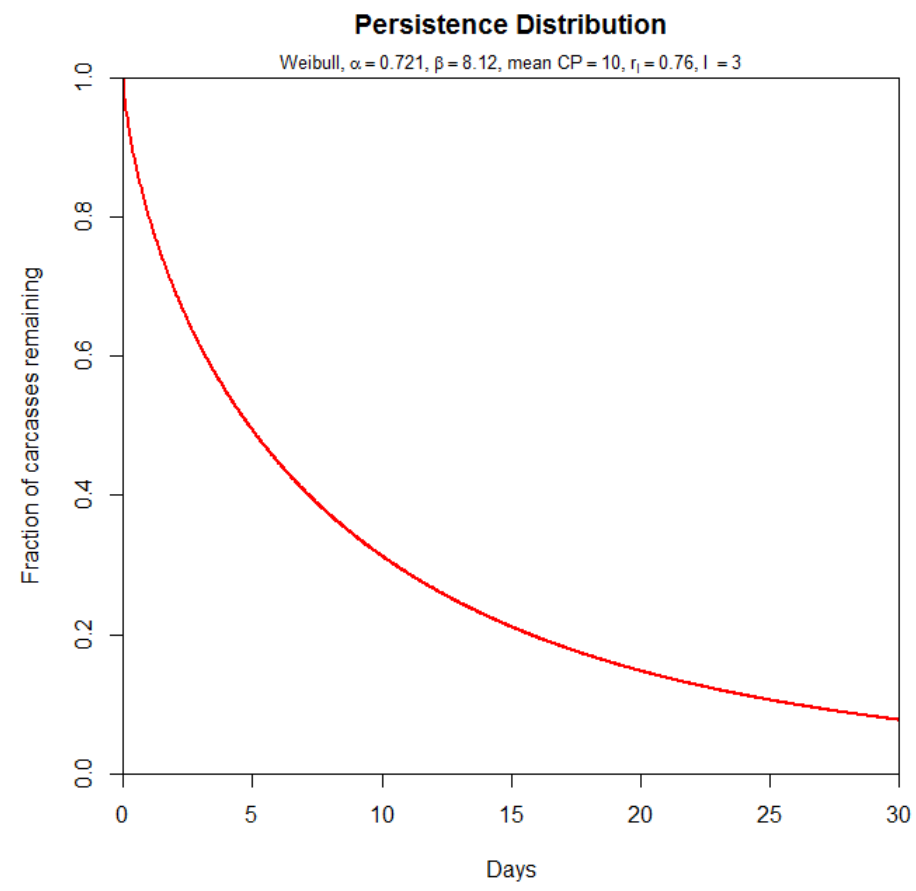

Figure 23. Graph generated by Evidence of Absence software showing user-selected persistence distribution. 


\subsection{Calculate}

The Calculate button graphs the detection probabilities or credibility levels as functions of the input parameters. It also saves the active parameter set for reloading the next time the module is opened.

\subsubsection{Design for Detection Probability}

With the default parameters for the Design Tradeoffs Module (fig. 21), Calculate will produce a graph showing design tradeoffs for search interval and search coverage for the given persistence distribution and search interval (fig. 24). The critical combinations of search interval and coverage that can be expected to achieve a $g$ of 0.25 are indicated by the bold white line in the graph.
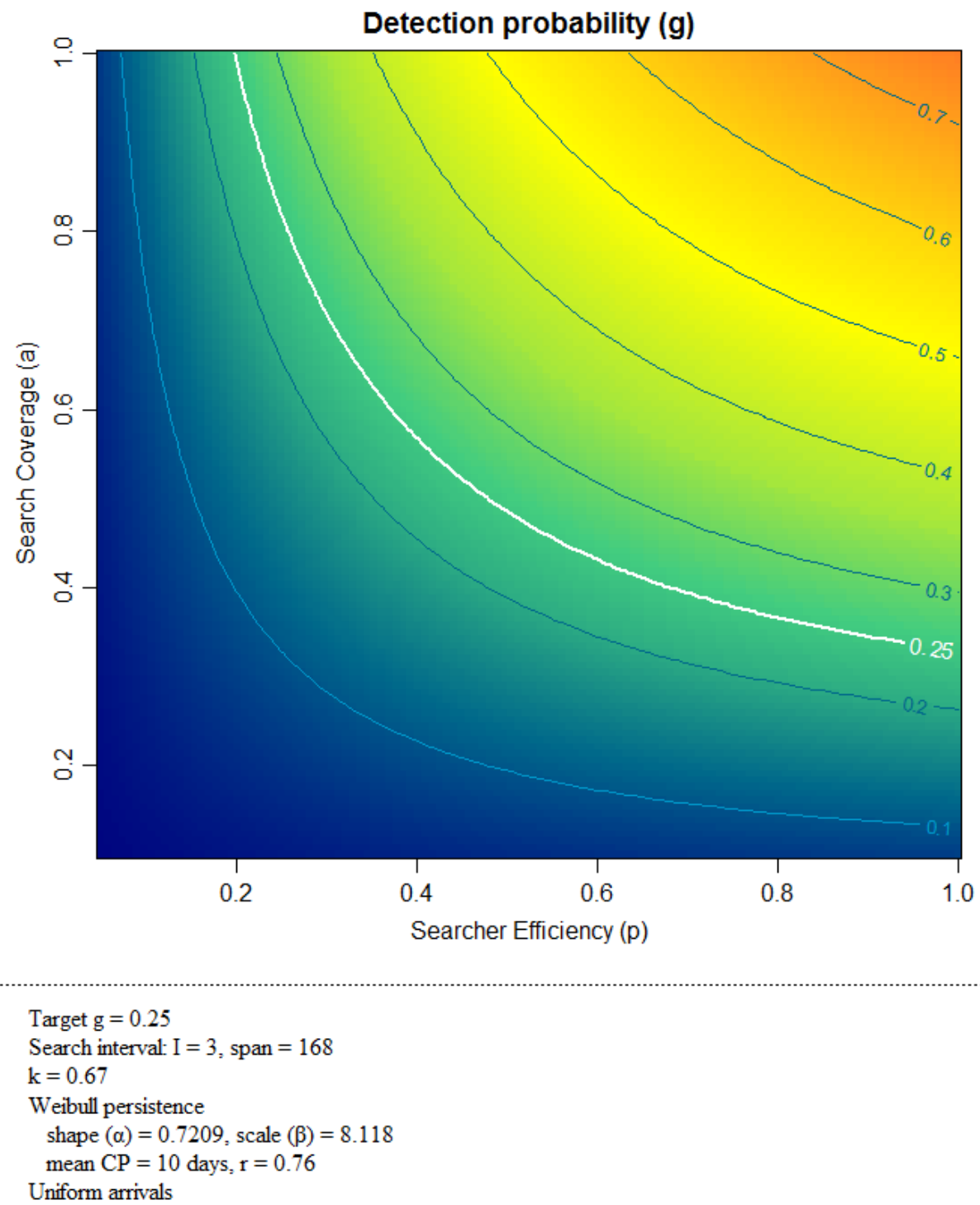

Figure 24. Graph generated by Evidence of Absence software showing tradeoffs between search coverage and searcher efficiency for $g$. 
The white line represents the combinations of search coverage and searcher efficiency that would be expected to result in an overall $g$ equal to the target. Any combination below the white line would result in a $g$ less than the target, and any combination above the white line would result in a $g$ greater than the target. For example, looking at the upper left portion of the graph, we see that with $100 \%$ search coverage, the target overall probability of detection of 0.25 can only be achieved if searcher efficiency is $>0.2$. The lower right portion of the graph shows that if searcher efficiency is $100 \%$, the target $g$ can only be achieved with search coverage $\geq 0.3$. A "goldilocks" solution might lie in having searcher efficiency of $\sim 0.4$ combined with a search coverage of about $\sim 0.55$.

This solution applies to the fixed interval of 3 days. The user may wish to explore other solutions in combination with search interval. By changing $I$ from Fixed to Variable, with a range from 3 to 28 days (both of which evenly divide the span of 168 days), a series of graphs similar to the previous one is produced (fig. 25), each representing the tradeoffs between searcher efficiency and search coverage for a different search interval.

As the search interval increases, the proportion of carcasses persisting decreases, requiring either higher searcher efficiency or higher search coverage to achieve the target overall detection probability. Nonetheless, the upper middle graph indicates that increasing the search coverage from 0.55 to 0.75 while keeping searcher efficiency at 0.4 will still allow the target detection probability to be achieved but with searches conducted only half as often $(I=6$ instead of 3$)$. The economic cost of increasing the searched area that comprises $75 \%$ of all carcasses can be balanced against the economic advantage of searching half as often. 


\section{Detection probability vs. searcher efficiency, coverage, and search interval}
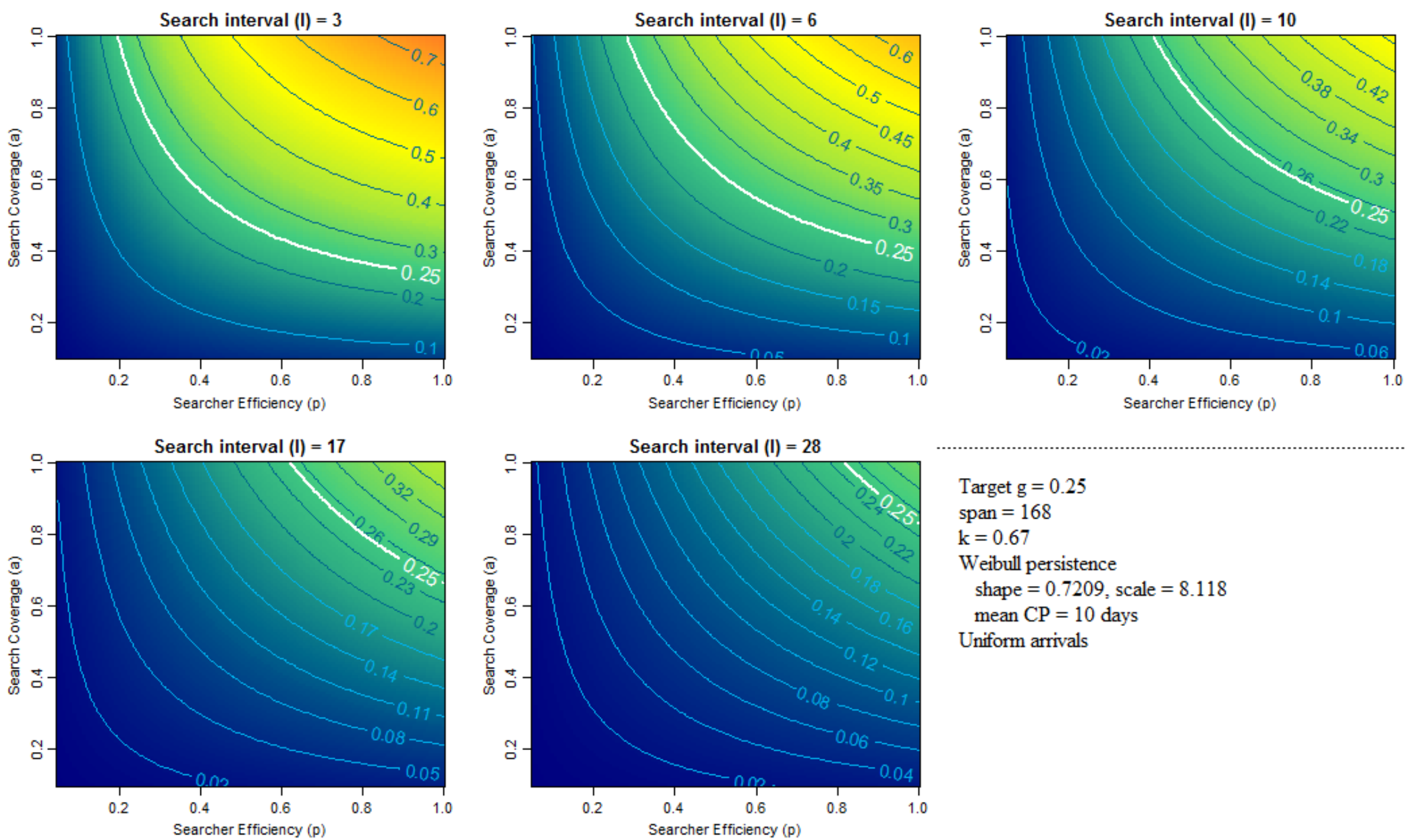

Target $\mathrm{g}=0.25$

span $=168$

$\mathrm{k}=0.67$

Weibull persistence

shape $=0.7209$, scale $=8.118$

mean $\mathrm{CP}=10$ days

Uniform arrivals

Figure 25. Graphs generated by Evidence of Absence software showing tradeoffs between search coverage and searcher efficiency for $g$, with $I$ varying. 


\subsubsection{Design for Credibility Level}

In some cases, there is a need to provide assurance at a set level of credibility $(1-\alpha)$ that no more than $\tau$ individuals of a species have been killed, even if 0 (or 1 or 2 or...) are discovered in the monitoring process. User inputs the threshold $(\tau)$, the target credibility level $(1-\alpha)$, and the critical carcass count $(X)$ for which the assurance is desired. For example, with the default data set (fig. 21), Calculate produces a graph showing design tradeoffs for search interval and search coverage for the given persistence time and search interval (fig. 26). The critical combination of search interval and coverage that can be expected to achieve a credible level of $1-\alpha$ is indicated by the bold line in the graph.

The bold line represents combinations of search coverage and searcher efficiency that would result in $100(1-\alpha) \%$ credibility level for asserting that $M \leq \tau$ given that $X=0$ carcasses are observed. Any combination below or to the left of the white line would result in lower credibility levels for asserting $M \leq \tau$, and combinations above or to the right of the white line would result in higher credibility levels for asserting $M \leq \tau$.

Again, the user may wish to explore other solutions in combination with search interval. By changing $I$ from Fixed to Variable, with a range from 3 to 28 days, a series of graphs similar to the previous one is produced (fig. 27), each representing the tradeoffs between searcher efficiency and search coverage for a different search interval.

As the search interval increases, more carcasses are scavenged before searchers can find them, and either higher searcher efficiency or higher search coverage is required to achieve the same level of credibility. For example, the upper left graph (fig. 27) indicates the target credibility level could be achieved with a search interval of 3 days with searcher efficiency of 0.4 and coverage area $\sim 0.45$. Alternatively, the upper right graph (fig. 27) indicates that target credibility level could be achieved with a search interval of 10 days if both searcher efficiency coverage area are $\geq 0.6$. The economic cost of increasing the searched area to comprise $60 \%$ of all carcasses and increasing the searcher efficiency within that area to 0.6 can be balanced against the economic advantage of searching one third as often. 


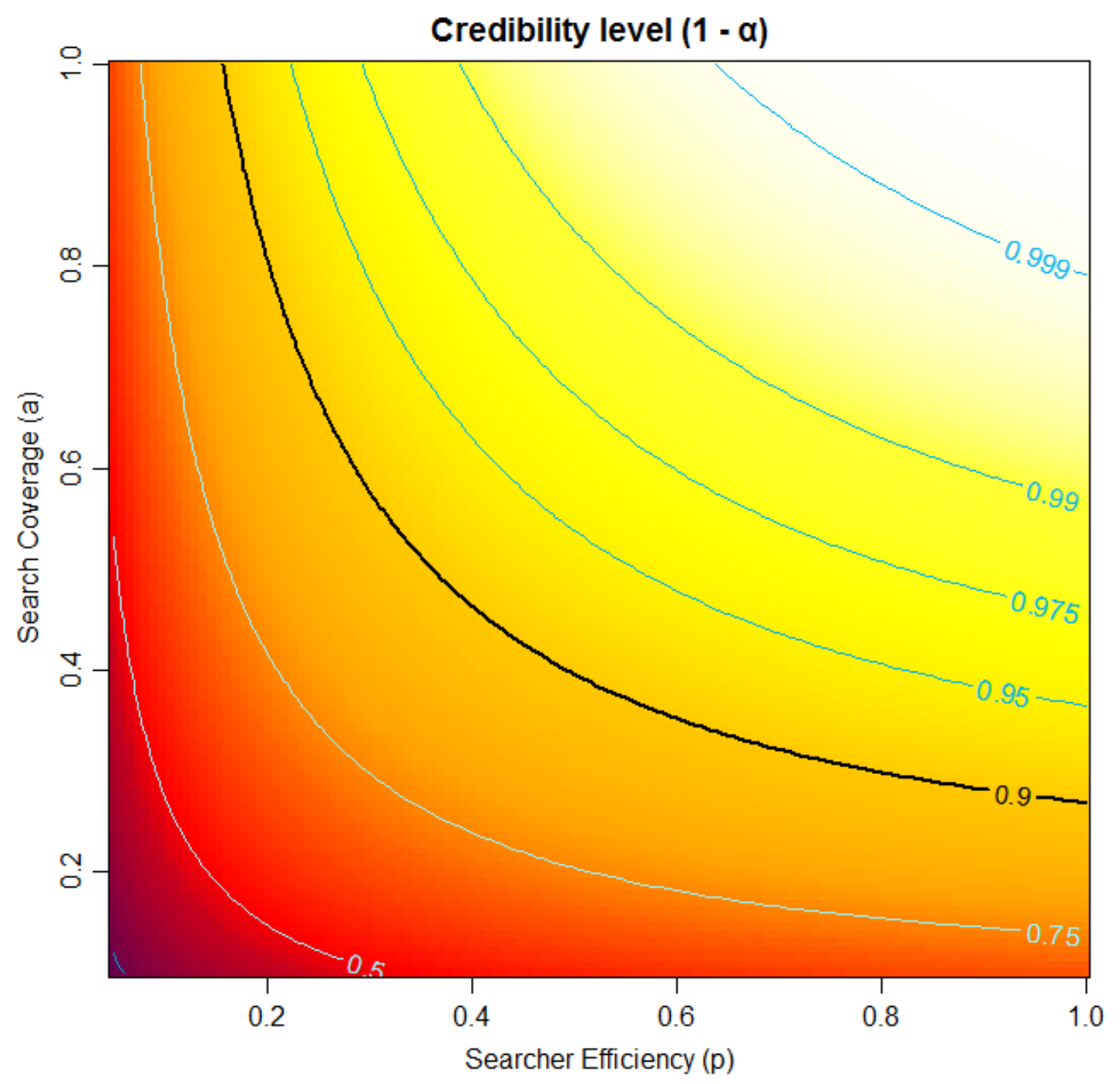

$1-\alpha=\mathrm{P}(\mathrm{M}<=5 \mid \mathrm{X}=0)$

Search interval: $I=3$, span $=168$

$\mathrm{k}=0.67$

Weibull persistence

shape $(\alpha)=0.7209$, scale $(\beta)=8.118$

mean $\mathrm{CP}=10$ days, $\mathrm{r}=0.76$

Uniform arrivals

Figure 26. Graph generated by Evidence of Absence software showing tradeoffs between search coverage and searcher efficiency for $1-\alpha$. 
Credibility level $(1-\alpha)$ vs. searcher efficiency, coverage, and search interval
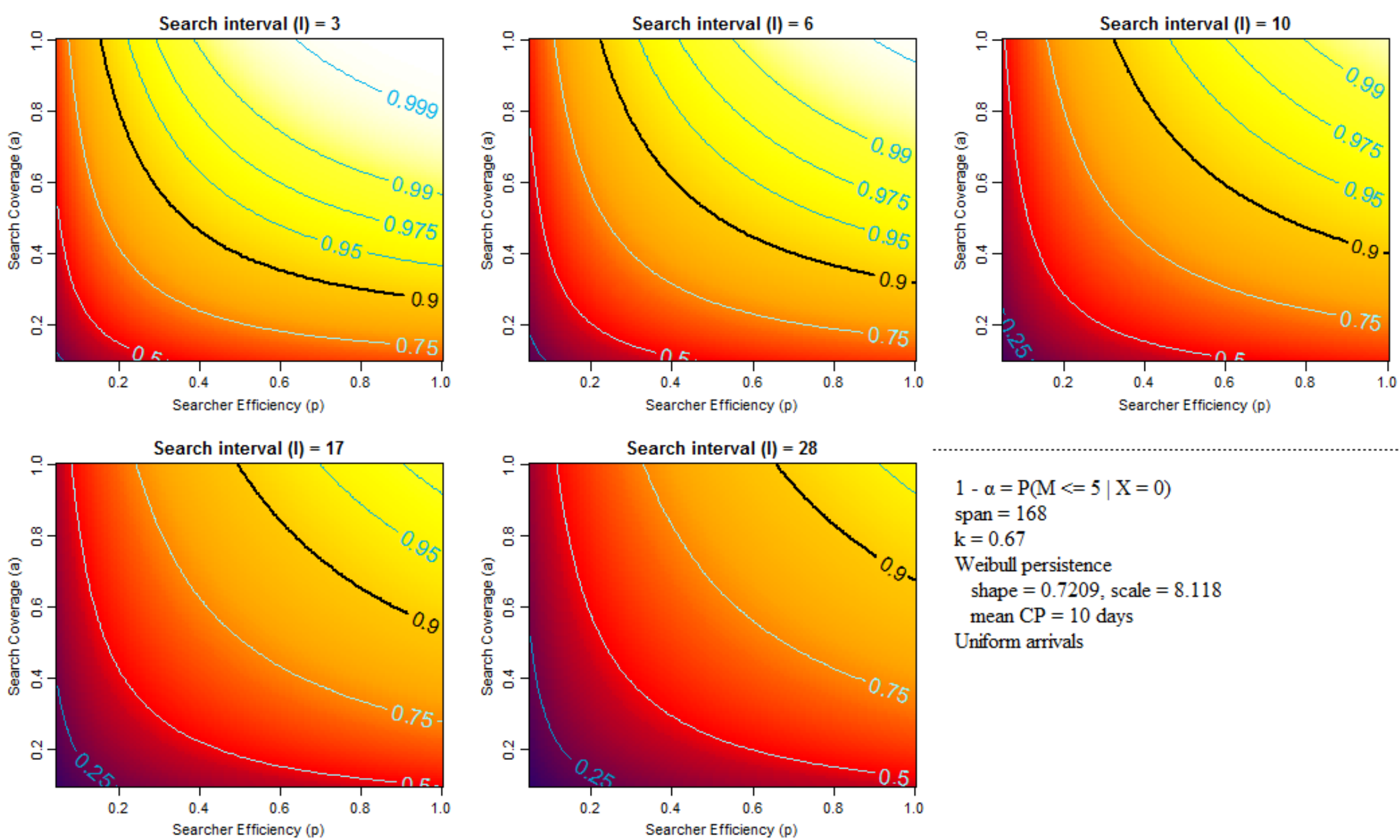

$1-\alpha=\mathrm{P}(\mathrm{M}<=5 \mid \mathrm{X}=0)$

$\operatorname{span}=168$

$\mathrm{k}=0.67$

Weibull persistence

shape $=0.7209$, scale $=8.118$

mean $\mathrm{CP}=10$ days

Uniform arrivals

Figure 27. Graphs generated by Evidence of Absence software showing tradeoffs between search coverage and searcher efficiency for $1-\alpha$, with variable $I$ values. 


\section{Scenario Explorer}

\subsection{Overview}

The scenario explorer provides tools for investigating the projected consequences of userdefined adaptive management regimes. The general framework is monitoring and adaptive management decision-making in the context of a long-term take permit (Dalthorp and Huso, 2015). The user defines the duration of the permit and the total permitted take. In addition, the user defines an assumed annual fatality rate and monitoring regime. Finally, the user defines parameters for a decision protocol for determining what adaptive management actions to take and the assumed effects of those actions in changing the fatality rates and carcass detection probabilities. EoA then simulates the projected number of fatalities and projected future constraints on wind farm operations induced by adaptive management.

\subsubsection{Long-Term Trigger}

[Reproduced with modification from Dalthorp and Huso, 2015]

Total take accumulates from year to year. Progress toward a long-term take limit of T is tracked using the Multiple Years module in the EoA software. Because actual cumulative take $(M)$ is not known, estimated cumulative total $\left(M^{*}\right)$ must be used instead. Exceedance of the long-term limit $\left(M^{*}>\mathrm{T}\right)$ triggers AMA to avoid further take. The value of $M^{*}$ is strongly tied to choice of required significance level $\alpha$, which must be agreed on before monitoring begins. Small values of $\alpha$ (for example, 0.1 or 0.2 ) give relatively strong assurance that take does not exceed the permitted limit at any given project, but triggering will tend to occur well before $M>\mathrm{T}$. A value of $\alpha=0.5$ results in more accurate tracking of actual fatality rates with triggering tending to occur after $M>\mathrm{T}$, because the trigger is not designed to prevent exceedance but to signal when exceedance has occurred.

As an example, suppose the long-term authorized take is $\mathrm{T}=60$ for a 30 -year permit. Ideally, if we knew exactly the number of fatalities each year, we could track the cumulative number of fatalities through the years and implement full-avoidance AMA when the cumulative total exceeded T. But in practice, the number of fatalities is estimated from carcass counts, after accounting for estimated detection probability, with varying degrees of certainty (fig. 28). A conservative approach using a small value of $\alpha$, for example, $\alpha=0.1$, would (1) give greater assurance that fatality rates do not exceed permitted levels, (2) provide a buffer against potential underestimation of fatality resulting from inadvertent mischaracterization of detection probability, and (3) provide a margin of safety against a full-avoidance AMA that is less than $100 \%$ effective. But it would also result in a higher likelihood of triggering well before the limit of 60 has been reached. If the factors that contribute to imperfect detection-most notably coverage (that is, the fraction of total carcasses that arrive in the search area during the monitoring period), carcass persistence, searcher efficiency, and change in searcher efficiency with carcass age - are properly accounted for and $g$ accurately reflects overall detection probability for the species of concern, then 0.5 will generally be the most accurate choice for $\alpha$. 


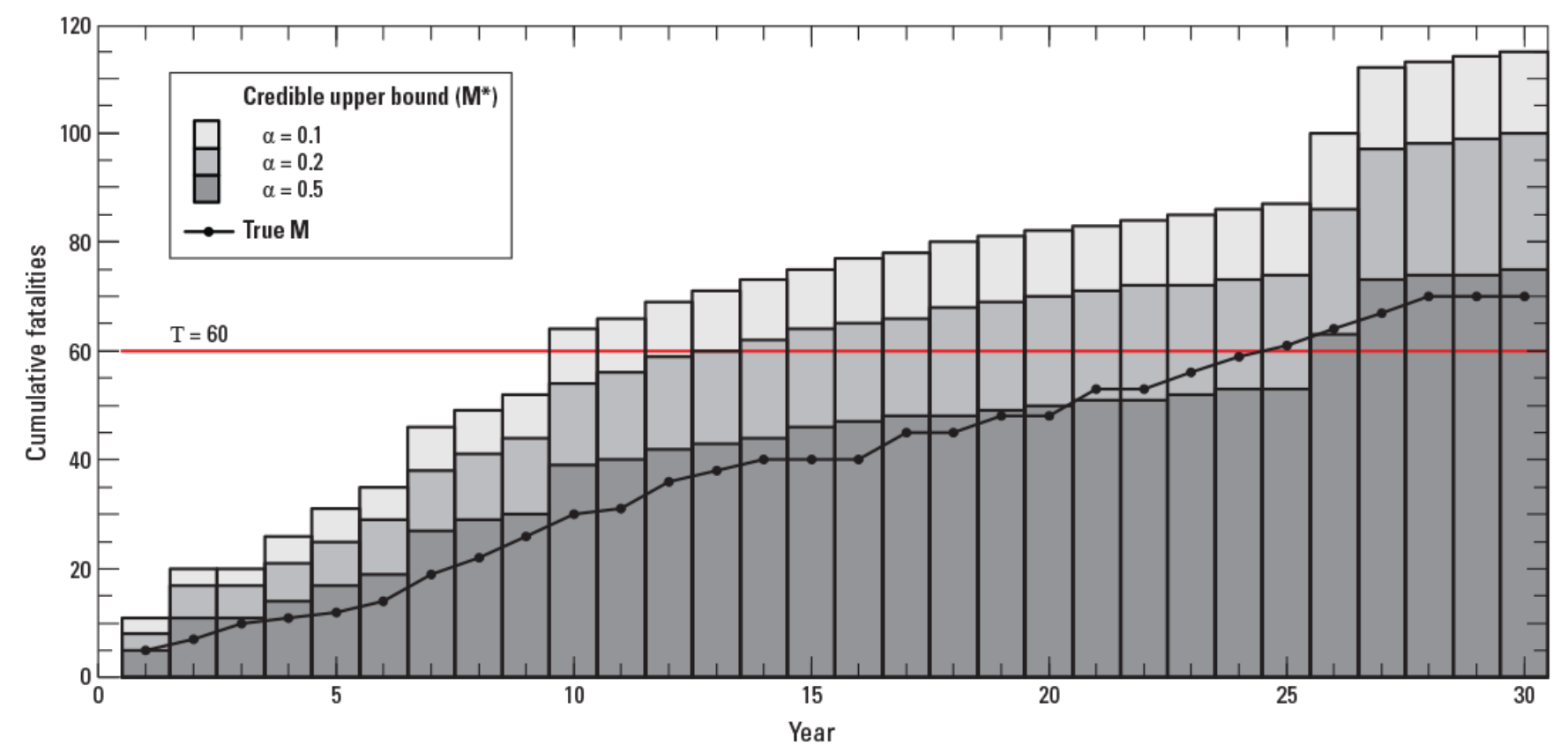

Figure 28. Graph generated by Evidence of Absence software showing operation of the long-term trigger. Simulated yearly fatalities were generated from a Poisson distribution with an annual rate of $\lambda=2$. Carcass counts were generated from the fatalities as binomial random variables with probability of success equal to the detection probability $g$, which was taken as 0.3 in the first 3 years and 0.08 in years $4-30$. Credible intervals were calculated using the Multiple Years module in the Evidence of Absence software. See table 1 for explanation of terms used here. (Reproduced from Dalthorp and Huso, 2015.)

In the scenario portrayed in figure 28 , the actual number of fatalities first exceeds $\mathrm{T}$ in year 25 , whereas he estimated number of fatalities $M^{*}$ with $\alpha=0.5$ first exceeds $\mathrm{T}$ to fire the long-term trigger in year 26. A value of $\alpha=0.2$ would result in triggering in year 14 when the actual cumulative mortality was 39 -well below the limit of 60 , providing some assurance that $\mathrm{T}$ had not been exceeded and some assurance that $\mathrm{T}$ will not be greatly exceeded if fatalities continue to accumulate after the AMA is implemented. An even more conservative approach would be to base $M^{*}$ on $\alpha=0.1$, which, in this example, would induce triggering in year 10 when the number of fatalities was 30 (fig. 28).

The long-term trigger (appendix G, section G.1) with $\alpha=0.5$ is designed to signal when take exceeds permitted limits, but it is not designed to prevent exceedance. Indeed, the long-term trigger does not guard against the possibility that the take permitted for the entire life of the project occurs within just a few years or give any indication when annual take rates are well above $\tau$. The conservation effect of a total take of T may be more severe if it occurs in a short period rather than being spread out over the course of the project.

In addition, the long-term trigger does not provide a mechanism for detecting changes in fatality rates over time. Finally, the AMAs associated with the long-term trigger may be costly. Advance warning of impending long-term triggers coupled with less onerous AMAs to prevent exceedance may be desirable. To remedy these limitations of relying strictly on a long-term trigger, a short-term trigger can work in concert with the long-term trigger to give advance warning of possible exceedance, to detect changes in fatality rates, and to provide a framework for incremental AMAs to prevent eventual exceedance. 


\subsubsection{Short-Term Trigger}

[Reproduced with modification from Dalthorp and Huso, 2015]

The short-term trigger (appendix G, section G.2) is designed to signal when the annual fatality rate $(\lambda)$ exceeds a given level $(\tau)$ over the course or one or a few years. The short-term trigger acts as a precaution against unexpectedly high fatality rates, as a warning signal that the long-term authorized take is likely to be exceeded unless additional measures are taken to reduce take rate, and as a mechanism to signal significant changes in fatality rates. In response to short-term trigger firing, incremental AMAs may be implemented to improve the precision of estimates by more intensive monitoring, to reduce take rates to bring them more in line with expectations, or to adjust permitted take levels to align with actual take and offset the increase in permitted take with additional mitigation.

Even though the average permitted annual take at a site, $\tau$, might be "correct" and reflect the true annual take rate, the actual number of fatalities that occur will not be exactly the same every year, due simply to natural variation and random chance. The short-term trigger is designed to allow for some annual variation in actual take and to guard against "hair-trigger" decision points. The trigger fires when the observed data (carcass counts combined with detection probabilities) are incompatible with the permitted rate. In other words, if it is too unlikely $(\leq \alpha)$ that the number of carcasses counted would be as high as observed if the true fatality rate really were in line with the permitted rate, then the short-term trigger would fire. The test is conducted each year on a running average basis (with the user defining the term or width of the window for the running average). If the total number of carcasses observed in any moving window is not compatible with what would be expected if the rate were equal to permitted level, the trigger fires.

The value of $\alpha$ is approximately the probability of the trigger firing when $\lambda=\tau$. Thus, smaller values of $\alpha$ result in a more sensitive trigger because they require stronger evidence to conclude $\lambda>\tau$. A small value of $\alpha$ (for example, $\alpha=0.01$ ) may be necessary to protect against the trigger firing unnecessarily when $\lambda \leq \tau$.

\subsubsection{Reversion Trigger}

[Reproduced with modification from Dalthorp and Huso, 2015]

If a facility is operating under constraints that are expected to reduce fatality rates by a factor of $\rho,(0<\rho \leq 1)$ compared with rates expected under operations free from the constraints (that is, the fatality rate would be $\lambda$ for operations without the constraints and $\lambda \rho$ with the constraints), then the reversion trigger (section J.3) is designed to signal when fatality rates are low enough so that removal of the operational constraints would not be likely to result in annual fatality rates that exceed $\tau$. One possible use of the reversion trigger would be to relax an initial prophylactic constraint if fatality rates are well below expected rates. For example, if, from the beginning of a project, turbines are required to be curtailed at wind speeds $<5.0 \mathrm{~m} / \mathrm{s}$ to minimize bat fatalities, the reversion trigger could be used to determine if fatality rates are low enough to allow unconstrained operations and still remain below the permitted rate. 
In particular, a previous operational constraint or restrictive AMA implemented to reduce fatalities by a factor of $\rho$ can be reversed when take rate is demonstrated to be lower than $\tau \rho$ at a credibility level of $1-\alpha$ according to a test on average rate over the years since the AMA was implemented. The rationale is that reversing an AMA with effect of $\rho$ would have the effect of increasing the fatality rate to $\lambda / \rho$, so an initial fatality rate of $\lambda \leq \tau \rho$ would result in a rate $\lambda / \rho \leq \tau$ after reversion.

\subsection{Parameters}

The scenario explorer provides a collection of tools for analyzing the potential effects of long term take permits for conservation and facility operations. The tool is useful for guiding choices of regulatory parameters (years in permit, total permitted take, and compliance criteria) in the drafting of multi-year ITPs. Parameters are entered manually using the scenario explorer form (fig. 29).

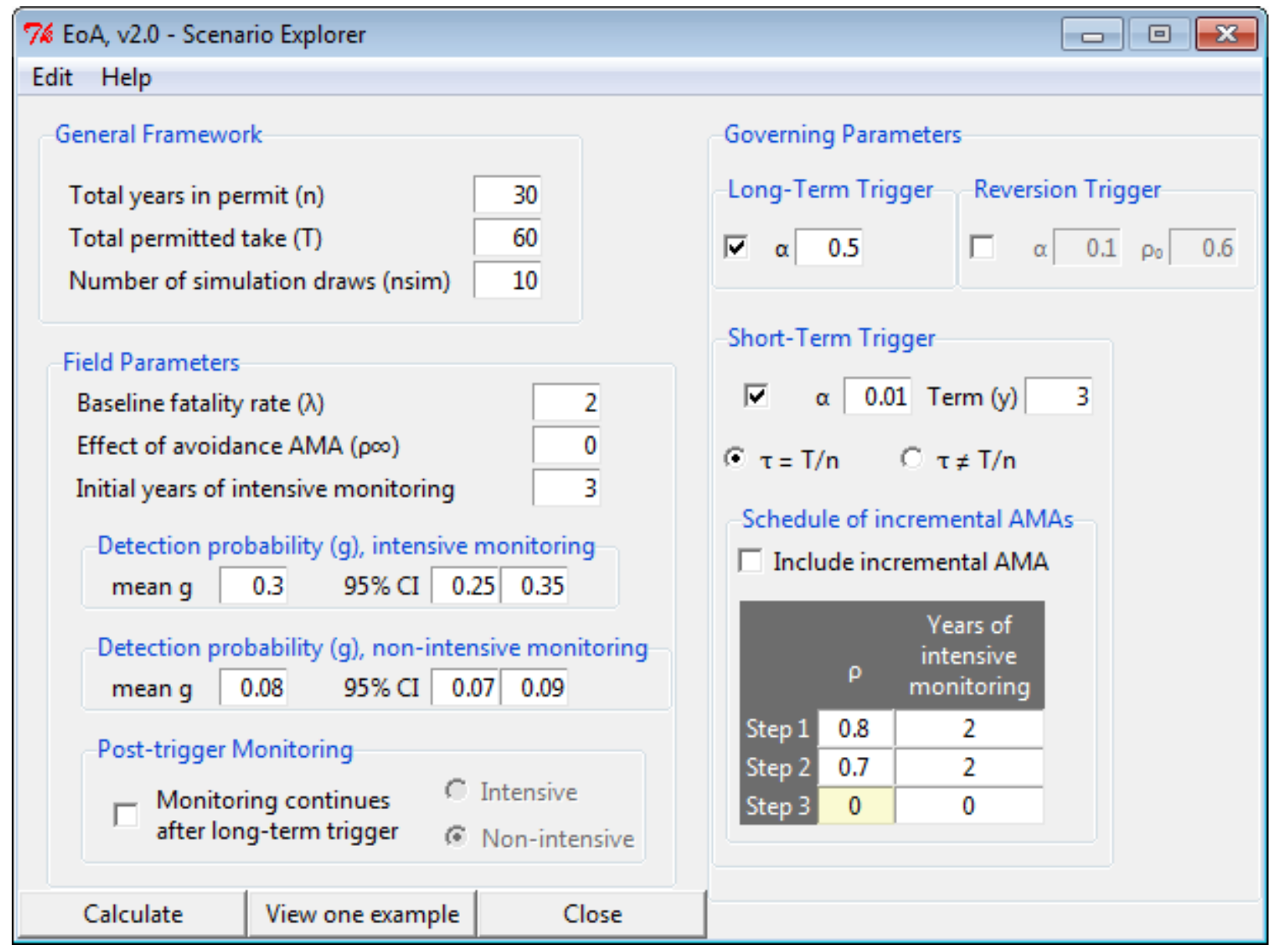

Figure 29. Screen capture of Scenario Explorer parameter input form with default data set. The default data set is a starting point for discussion and creating examples and should not interpreted in any way as "recommended" or "typical." 


\subsubsection{General Framework}

For the general framework of the simulations, the user inputs the total number of years in the permit, the total permitted take over the full term of the permit, and the number of simulation draws.

Simulation results are highly unstable when the number of simulation draws (nsim) is small and should not be considered reliable for nsim $<1000$. However, simulations that involve incremental AMAs, a reversion trigger, or post-trigger intensive monitoring (fig. 30) run slowly. It may take a number of attempts and a period of trial and error to find a parameter set that properly defines the desired scenario. In these cases, it may be desirable to start with $n$ sim $=10$ until you are satisfied with the parameter set, and then boost the number of simulation draws to produce more accurate figures. Simulations that do not involve incremental AMAs, a reversion trigger, or post-trigger intensive monitoring run hundreds of times faster, and nsim can be set at 1,000 or 10,000 for these scenarios without concern about long wait times.

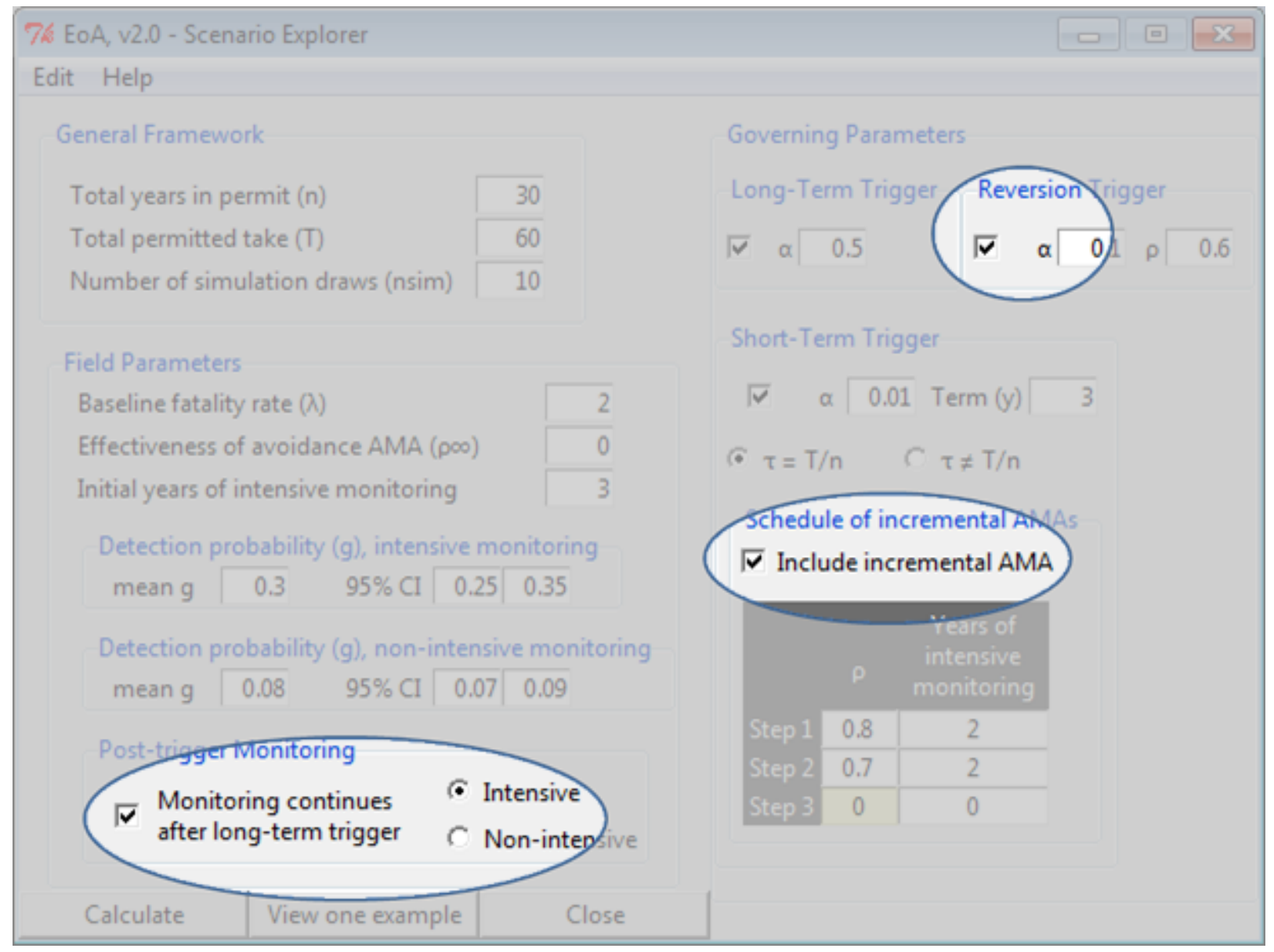

Figure 30. Screen capture of Scenario Explorer parameter options with potentially slow simulation speeds. 


\subsubsection{Field Parameters}

The baseline mortality rate $(\lambda)$ is the expected number of fatalities generated each year at the start of the permit term. The mortality rate is held constant in subsequent years unless altered by adaptive management actions (AMAs), which would change the mortality rate by a factor of $\rho$ (defined by the user).

In some regulatory scenarios, after the permitted take is exceeded, an AMA to avoid future take must be implemented. This avoidance AMA is assumed to change mortality by a factor of $\rho_{\infty}$. For example, $\rho_{\infty}=0$ would imply that the AMA would reduce the mortality rate to $\lambda \rho_{\infty}=0$. An avoidance AMA that is not entirely effective may only reduce the mortality rate by $90 \%$, and $\rho_{\infty}$ would be set to 0.1 .

The monitoring regime is defined in terms of the overall detection probability, $g$, which may be set for more intensive monitoring in the initial years of the permit followed by less intensive monitoring in subsequent years. Alternatively, the monitoring regime can be held constant by setting the Initial years of intensive monitoring equal to the total years in the permit. The user has the option of whether to continue monitoring after the long-term trigger is fired and to set the post-trigger monitoring intensity.

\subsubsection{Governing Parameters}

The governing parameters define long-term, short-term, and reversion triggers, which are the criteria for determining, respectively, exceedance of the permitted total take, excessively high average mortality rates that signal a change in mortality rates or eventual exceedance of permitted take, and low rates that would allow safe removal of previous operational constraints implemented to reduce mortality rates.

The long-term trigger is designed to signal when the permitted take $(\mathrm{T})$ has been exceeded. The sensitivity of the trigger can be adjusted via the $\alpha$ parameter, with smaller values of $\alpha$ defining a more sensitive trigger. More specifically, the long-term trigger signals when $M^{*}>\mathrm{T}$ or, equivalently, $P(M>\mathrm{T})>\alpha$ according to the posterior distribution of $M \mid(X, \hat{g})$. A value of $\alpha=0.5$ yields the most accurate estimate of $M$ and triggering that, on average, occurs when $M$ first exceeds $\mathrm{T}$. Smaller values of $\alpha$ in the long-term trigger result in greater $M^{*}$ and, in most cases, triggering before $M>\mathrm{T}$. Likewise, larger values of $\alpha$ yield smaller $M^{*}$ s with triggering typically occurring several years after $M>\mathrm{T}$. 
The short-term trigger tests whether the average annual mortality rate $(\lambda)$ has exceeded some value of concern $(\tau)$ over a term of the past $y$ years. Setting $\tau=\mathrm{T} / n$ tests whether the annual rate is consistent with a long-term rate of $\mathrm{T}$, and triggering would provide an early warning that the long-term rate is likely to be exceeded before the end of the permit term unless actions are taken to reduce the take rate or increase the take limit. Users also have the option of setting $\tau$ to values other than $\mathrm{T} / n$ if desired. The value of $\alpha$ governs the sensitivity of the trigger. For example, triggering when $\alpha=0.01$ indicates that there is approximately a $1 \%$ chance that we would have observed the sequence of counts we did, if $\lambda=\tau$. Larger values of $\alpha$ in the short-term trigger correspond to greater sensitivity and less certainty that $\lambda>\tau$ at triggering. Because the short-term rate is tested year after year, even if $\lambda \leq \tau$ the probability of erroneously triggering (type I error) at least once over the course of an entire project may be substantially greater than triggering in any particular term of $y$ years. Thus, there is an elevated risk of type I errors with the short-term trigger, and that risk can be managed by choosing a small value for $\alpha$ (like $\alpha=0.01$ ) and using an incremental approach to adaptive management to define a schedule of increasingly restrictive constraints designed to reduce mortality rates and/or increase monitoring requirements each time the short-term trigger is fired.

Users may select the option to include incremental AMAs. For example, a 3-step schedule of incremental AMAs might require action to reduce the mortality rate by $20 \%$ to $0.8 \lambda$ and to add 2 years of intensive monitoring after the first time the short-term trigger fires, to reduce the mortality rate to $0.7 \lambda$ with another 2 years of intensive monitoring after the second triggering, and avoidance AMA after the third firing. Steps may be added or removed from the schedule by pressing $<\mathrm{Ctrl}+\mathrm{a}>$ or $<\mathrm{Ctrl}+\mathrm{d}>$ when the cursor is in the AMA table. If the option to include incremental AMA is not selected, the simulations tally the number of times the short-term trigger fires but do not alter the mortality rate or monitoring intensity in response to triggering.

The reversion trigger tests whether the mortality rate is low enough so that a current operational constraint to reduce mortality can be removed while keeping the resulting rate below $\tau=\mathrm{T} / n$. For example, suppose curtailment of turbines at wind speeds less than $5 \mathrm{~m} / \mathrm{s}$ reduces the mortality rate to $\rho_{0}=60 \%$ of the uncurtailed rate and that at the start of the permit term, turbines are curtailed as a prophylactic measure to minimize mortality. If the observed mortality rate after some years of monitoring is significantly smaller than $\tau \rho_{0}$, then after removing the constraint and increasing the mortality rate by a factor of $1 / \rho_{0}$, the resulting mortality rate would still be less than $\tau$. The reversion trigger tests whether a constraint with effect $\rho_{0}$ can be reversed with the subsequent mortality rate significantly less than $\tau$ (with significance level of $\alpha$ ). 


\subsection{Simulations}

The scenario explorer tracks fatalities, mortality estimates, and adaptive management actions for nsim simulated projects year by year through the entire term of the permit under the conditions defined by the parameters entered by the user. Two options for simulation are to View one example or

Calculate, which summarizes results from nsim simulated projects (fig. 31).

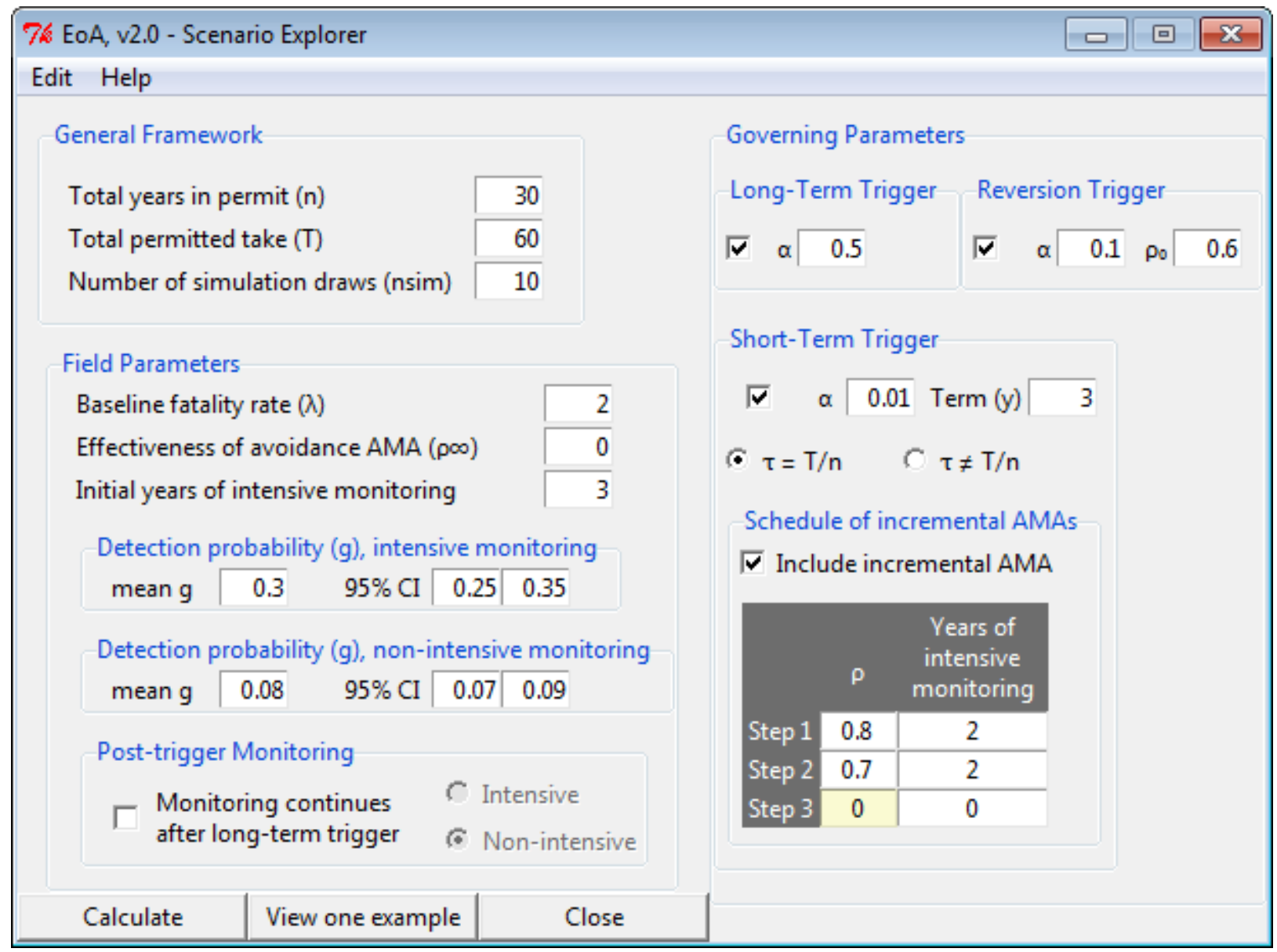

Figure 31. Screen capture of Scenario Explorer with default parameters but with "Reversion Trigger" and "Include incremental AMA" options selected. 


\subsubsection{View One Example}

The tool to View one example tracks the number of fatalities, the mortality estimates, and management conditions for one simulated project through the $n$ years of the project term. The actions of the long-term, short-term, and reversion triggers are illustrated for three simulated projects using the tool (figs. 32-34). The three projects represent different random outcomes of the scenario described by the parameter set shown in figure 31. The total permitted take is $\mathrm{T}=60$ over the course of a 30 -year permit. The baseline fatality rate is $\lambda=2$ per year, so the expected take would be $30 \cdot 2=60$. However, the number of fatalities is a random variable, and the total will rarely be exactly 60 but will usually be somewhat more or less than 60 .

In the first simulated project (fig. 32), the cumulative number of fatalities $(M)$ reaches 61 in year 25. The estimated cumulative totals $\left(M^{*}\right)$ closely track the actual totals, and $M^{*}$ crosses the $\mathrm{T}=60$ threshold in year 26. At that point, the long-term trigger fires (that is, estimated mortality $M^{*}>\mathrm{T}$ for $\alpha=0.5$ ), avoidance AMA is implemented, and the mortality rate is reduced to $\rho_{\infty} \lambda=0$ in future years. Accumulation of fatalities ceases, and the total remains constant at 66 until the end of the permit term. To explore the consequences of avoidance AMA not reaching 100\% effectiveness, $\rho_{\infty}$ can be set to some non-zero value like 0.10 or 0.05 to reflect $90 \%$ or $95 \%$ effectiveness. Fatalities would still accumulate after the long-term trigger fires but at a greatly reduced rate.

The "cumulative $g$ " is the probability of observing a carcass that arrived in the current year or a previous year. For example, in year 4 non-intensive monitoring yielded $g=0.08$, so there is only an $8 \%$ chance of observing a carcass that arrives in that year. However, the probability of observing a carcass that arrives at any point in the first 4 years would be 0.245 , which is the average probability of detecting a carcass in years $1-4$. In this scenario, the overall cumulative detection probability gradually declines as more and more carcasses accumulate in years with low detection probabilities. When no new carcasses are observed, the estimated cumulative total number of carcasses $\left(M^{*}\right)$ increases as cumulative $g$ decreases, but the increase is much more gradual than the increase in actual fatalities $(M)$. When new carcasses are found (years $1,2,5,9,19$, and 26), $M^{*}$ leaps by approximately the number of carcasses found in that year divided by the cumulative $g$.

In the second simulated project (fig. 33), fatalities accumulated rapidly in the initial years (reaching 29 in year 9)an average of 3.2 per year, which is substantially greater than expected $\lambda=2$. The short-term trigger fired $(P(\lambda>\tau)>0.99$, which is 1 minus the short-term $\alpha)$ as the 3 carcasses discovered in the previous 3 years gave strong indication that actual fatality rate over the previous 3 years exceeded the permitted rate of 2 . In response, the first step in incremental AMA was triggered, reducing the mortality rate to $\rho \lambda=1.6$ and requiring monitoring at $g=0.3$ for the next two years. No carcasses were found during those two years, and the estimated cumulative fatalities actually decreased as the cumulative overall detection probability increased. The actual total number of fatalities did not decrease; however, the quality of information available about the cumulative total improved, and the new information suggests that the cumulative total was less than previously thought. Mortality continued at a reduced rate until year 27 , when the cumulative number of fatalities reached 54 but the long-term trigger fired as the estimated total exceeded 60. 


\begin{tabular}{|c|c|c|c|c|c|c|c|c|c|c|c|c|c|c|c|c|c|}
\hline \multicolumn{3}{|c|}{ 76. EoA, v2.0 - Scenario Explorer, One Example } & & & & & & & & & & & & \multicolumn{4}{|c|}{\begin{tabular}{|l|l|} 
& 0 \\
\end{tabular}} \\
\hline & & & \multicolumn{3}{|c|}{ Output: } & \multicolumn{3}{|c|}{ Annual } & \multicolumn{3}{|c|}{ Cumulative } & \multirow{2}{*}{$\begin{array}{c}\text { Long-term } \\
\qquad \mathrm{M}^{*}\end{array}$} & \multirow{2}{*}{$\begin{array}{l}\text { Reversion } \\
p(\lambda<\tau \rho)\end{array}$} & \multicolumn{4}{|c|}{ Short-term } \\
\hline \multicolumn{2}{|c|}{ Input parameters } & & Year & M & $\mathrm{x}$ & $\mathrm{g}$ & $\rho$ & $\lambda$ & M & $\mathrm{x}$ & $\mathrm{g}$ & & & M & $\mathrm{x}$ & $\mathrm{g}$ & $p(\lambda>\tau)$ \\
\hline \multicolumn{2}{|c|}{ Permit years } & 30 & 1 & 3 & 1 & 0.3 & 1 & 2 & 3 & 1 & 0.3 & 4 & 0.131 & 3 & 1 & 0.3 & 0.314 \\
\hline \multicolumn{2}{|c|}{ Total permitted take (T) } & 60 & 2 & 5 & 1 & 0.3 & 1 & 2 & 8 & 2 & 0.3 & 7 & 0.08 & 8 & 2 & 0.3 & 0.611 \\
\hline \multicolumn{2}{|c|}{ Baseline fatality rate $(\lambda)$} & 2 & 3 & 4 & 0 & 0.3 & 1 & 2 & 12 & 2 & 0.3 & 7 & 0.173 & 12 & 2 & 0.3 & 0.61 \\
\hline \multicolumn{2}{|c|}{$\mathrm{g}$ (intensive) } & $0.3(0.25,0.35)$ & 4 & 4 & 0 & 0.08 & 1 & 2 & 16 & 2 & 0.245 & 9 & 0.201 & 13 & 1 & 0.227 & 0.48 \\
\hline \multicolumn{2}{|c|}{$g$ (non-intensive) } & $0.08(0.07,0.09)$ & 5 & 4 & 2 & 0.08 & 1 & 2 & 20 & 4 & 0.212 & 19 & 0.02 & 12 & 2 & 0.153 & 0.877 \\
\hline \multicolumn{2}{|c|}{ long-term trigger $(\alpha)$} & 0.5 & 6 & 1 & 0 & 0.08 & 1 & 2 & 21 & 4 & 0.19 & 22 & 0.026 & 9 & 2 & 0.08 & 0.966 \\
\hline \multicolumn{2}{|c|}{ long-term trigger ( $p \infty)$} & 0 & 7 & 1 & 0 & 0.08 & 1 & 2 & 22 & 4 & 0.174 & 24 & 0.033 & 6 & 2 & 0.08 & 0.966 \\
\hline \multicolumn{2}{|c|}{ short-term trigger $(\tau)$} & 2 & 8 & 4 & 0 & 0.08 & 1 & 2 & 26 & 4 & 0.162 & 25 & 0.041 & 6 & 0 & 0.08 & 0.328 \\
\hline \multicolumn{2}{|c|}{ short-term trigger $(\alpha)$} & 0.01 & 9 & 2 & 1 & 0.08 & 1 & 2 & 28 & 5 & 0.153 & 33 & 0.014 & 7 & 1 & 0.08 & 0.811 \\
\hline \multicolumn{2}{|c|}{ short-term trigger (term) } & 3 & 10 & 0 & 0 & 0.08 & 1 & 2 & 28 & 5 & 0.146 & 35 & 0.018 & 6 & 1 & 0.08 & 0.811 \\
\hline \multicolumn{2}{|c|}{ Incremental AMAs: } & $\rho$, monitoring & 11 & 3 & 0 & 0.08 & 1 & 2 & 31 & 5 & 0.14 & 37 & 0.022 & 5 & 1 & 0.08 & 0.811 \\
\hline \multicolumn{2}{|c|}{ step 1} & $0.8,2$ & 12 & 1 & 0 & 0.08 & 1 & 2 & 32 & 5 & 0.135 & 38 & 0.027 & 4 & 0 & 0.08 & 0.328 \\
\hline \multicolumn{2}{|l|}{ step 2} & $0.7,2$ & 13 & 5 & 0 & 0.08 & 1 & 2 & 37 & 5 & 0.131 & 39 & 0.033 & 9 & 0 & 0.08 & 0.328 \\
\hline \multicolumn{2}{|c|}{ step 3} & 0,0 & 14 & 0 & 0 & 0.08 & 1 & 2 & 37 & 5 & 0.127 & 40 & 0.039 & 6 & 0 & 0.08 & 0.328 \\
\hline Reversion tric & $\operatorname{ger}(\alpha)$ & 0.1 & 15 & 0 & 0 & 0.08 & 1 & 2 & 37 & 5 & 0.124 & 41 & 0.046 & 5 & 0 & 0.08 & 0.328 \\
\hline Reversion tric & $\operatorname{ger}(\rho)$ & 0.6 & 16 & 3 & 0 & 0.08 & 1 & 2 & 40 & 5 & 0.121 & 42 & 0.053 & 3 & 0 & 0.08 & 0.328 \\
\hline & & & 17 & 5 & 0 & 0.08 & 1 & 2 & 45 & 5 & 0.119 & 43 & 0.062 & 8 & 0 & 0.08 & 0.328 \\
\hline & & & 18 & 1 & 0 & 0.08 & 1 & 2 & 46 & 5 & 0.117 & 44 & 0.071 & 9 & 0 & 0.08 & 0.328 \\
\hline & & & 19 & 2 & 1 & 0.08 & 1 & 2 & 48 & 6 & 0.115 & 53 & 0.03 & 8 & 1 & 0.08 & 0.811 \\
\hline & & & 20 & 1 & 0 & 0.08 & 1 & 2 & 49 & 6 & 0.113 & 54 & 0.035 & 4 & 1 & 0.08 & 0.811 \\
\hline & & & 21 & 1 & 0 & 0.08 & 1 & 2 & 50 & 6 & 0.111 & 55 & 0.041 & 4 & 1 & 0.08 & 0.811 \\
\hline & & & 22 & 3 & 0 & 0.08 & 1 & 2 & 53 & 6 & 0.11 & 56 & 0.047 & 5 & 0 & 0.08 & 0.328 \\
\hline & & & 23 & 1 & 0 & 0.08 & 1 & 2 & 54 & 6 & 0.109 & 56 & 0.054 & 5 & 0 & 0.08 & 0.328 \\
\hline & & & 24 & 4 & 0 & 0.08 & 1 & 2 & 58 & 6 & 0.108 & 57 & 0.061 & 8 & 0 & 0.08 & 0.328 \\
\hline & & & 25 & 3 & 0 & 0.08 & 1 & 2 & 61 & 6 & 0.106 & 58 & 0.069 & 8 & 0 & 0.08 & 0.328 \\
\hline & & & 26 & 5 & 1 & 0.08 & 1 & 2 & 66 & 7 & 0.105 & 68 & NA & 12 & 1 & 0.08 & 0.811 \\
\hline & & & 27 & 0 & 0 & 0 & 0 & 0 & 66 & 7 & NA & NA & NA & NA & NA & NA & NA \\
\hline & & & 28 & 0 & 0 & 0 & 0 & 0 & 66 & 7 & NA & NA & NA & NA & NA & NA & NA \\
\hline & & & 29 & 0 & 0 & 0 & 0 & 0 & 66 & 7 & NA & NA & NA & NA & $\mathrm{NA}$ & NA & NA \\
\hline & & & 30 & 0 & 0 & 0 & 0 & 0 & 66 & 7 & NA & NA & NA & NA & NA & NA & NA \\
\hline Edit/Close & $\begin{array}{l}\text { Save to file } \\
\text { (.txt) }\end{array}$ & $\begin{array}{l}\text { Generate } \\
\text { Another }\end{array}$ & & & & & & & & & & & & & & & \\
\hline
\end{tabular}

Figure 32. Screen capture of Scenario Explorer, one example (long-term trigger). Cumulative mortality $(M)$ first exceeds the threshold of $\mathrm{T}=60$ in year 25 (red font in the column for cumulative $M$ ). The following year the longterm trigger signals exceedance as $M^{*}$ exceeds $\mathrm{T}$ (red cell in the $M^{*}$ column). 


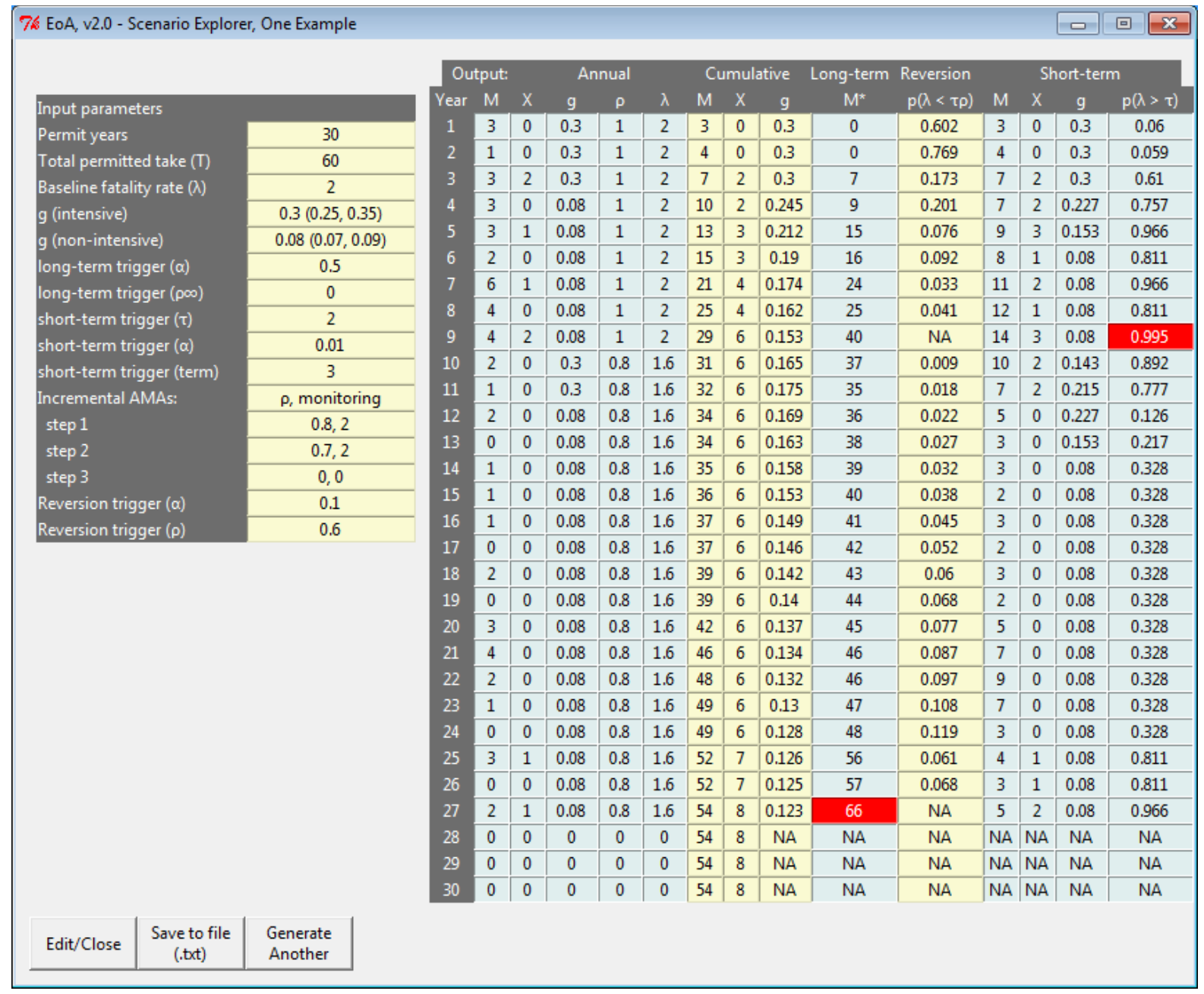

Figure 33. Screen capture of Scenario Explorer, one example (short-term trigger). The 3-year running total carcass count was 3 in year 9 ( $X$ in the "Short-term" area of the table), which, when combined with a low detection rate over these 3 years, was high enough to fire the short-term trigger (red cell in the right-hand column) because $P(\lambda>$ $\tau)=0.995>1-\alpha$. After trigger, AMA reduces future mortality rate by a factor of $\rho=0.8$, as governed by step 1 in the schedule of incremental AMAs. In addition, 2 years of intensive monitoring are required after triggering (annual $g$ increases to 0.3 for years 10 and 11).

In the third simulated project (fig. 34), there were only 6 fatalities in the first 6 years - many fewer than the expected $6 \lambda=12$. No carcasses were found in the searches, and the reversion trigger fired. The resulting AMA increased the mortality rate to 3.33, and fatalities accumulated rapidly for several years. In year 13, the short-term trigger detected the high mortality rate, and AMAs returned the mortality rate to its initial level, $\lambda=2$. By year $25, M^{*}$ reached 61 , and the long-term trigger correctly signaled that the permitted take rate had been exceeded. 
The examples discussed in figures 32-34 were specially selected for illustration. Repeated use of the Generate Another button will show that a wide variety of patterns are possible and that the reversion and short-term triggers do not fire often for this scenario. The long-term trigger fires more frequently, but usually the triggering is near the end of the permit term. More precise summary statistics on triggering and total fatalities can be obtained via simulating many such projects and cataloging the results. This can be done automatically via the Calculate button in the Scenario Explorer window (after closing the Single Example window).

\begin{tabular}{|c|c|c|c|c|c|c|c|c|c|c|c|c|c|c|c|c|c|}
\hline \multicolumn{3}{|c|}{ 76. EoA, v2.0 - Scenario Explorer, One Example } & & & & & & & & & & & & \multicolumn{4}{|c|}{\begin{tabular}{|l|l} 
& 0 \\
\end{tabular}} \\
\hline & & & \multicolumn{3}{|c|}{ Output: } & \multicolumn{3}{|c|}{ Annual } & \multicolumn{3}{|c|}{ Cumulative } & \multirow{2}{*}{$\begin{array}{c}\text { Long-term } \\
\mathrm{M}^{\star}\end{array}$} & \multirow{2}{*}{$\begin{array}{l}\text { Reversion } \\
p(\lambda<\tau \rho)\end{array}$} & \multicolumn{4}{|c|}{ Short-term } \\
\hline \multicolumn{3}{|c|}{ Input parameters } & Year & M & $\mathrm{x}$ & $g$ & $\rho$ & $\lambda$ & M & $x$ & $\mathrm{~g}$ & & & M & $\mathrm{x}$ & $\mathrm{g}$ & $p(\lambda>\tau)$ \\
\hline \multicolumn{2}{|c|}{ Permit years } & 30 & 1 & 0 & 0 & 0.3 & 1 & 2 & 0 & 0 & 0.3 & 0 & 0.602 & 0 & 0 & 0.3 & 0.06 \\
\hline \multicolumn{2}{|c|}{ Total permitted take $(T)$} & 60 & 2 & 1 & 0 & 0.3 & 1 & 2 & 1 & 0 & 0.3 & 0 & 0.769 & 1 & 0 & 0.3 & 0.059 \\
\hline \multicolumn{2}{|c|}{ Baseline fatality rate $(\lambda)$} & 2 & 3 & 3 & 0 & 0.3 & 1 & 2 & 4 & 0 & 0.3 & 0 & 0.858 & 4 & 0 & 0.3 & 0.059 \\
\hline \multicolumn{2}{|c|}{ g (intensive) } & $0.3(0.25,0.35)$ & 4 & 1 & 0 & 0.08 & 1 & 2 & 5 & 0 & 0.245 & 0 & 0.874 & 5 & 0 & 0.227 & 0.126 \\
\hline \multicolumn{2}{|c|}{$g$ (non-intensive) } & $0.08(0.07,0.09)$ & 5 & 1 & 0 & 0.08 & 1 & 2 & 6 & 0 & 0.212 & 0 & 0.889 & 5 & 0 & 0.153 & 0.217 \\
\hline \multicolumn{2}{|c|}{ long-term trigger $(\alpha)$} & 0.5 & 6 & 0 & 0 & 0.08 & 1 & 2 & 6 & 0 & 0.19 & 1 & 0.902 & 2 & 0 & 0.08 & 0.328 \\
\hline \multicolumn{2}{|c|}{ long-term trigger $(\rho \infty)$} & 0 & 7 & 3 & 0 & 0.08 & 1.67 & 3.33 & 9 & 0 & 0.166 & 1 & 0.905 & 4 & 0 & 0.08 & 0.328 \\
\hline \multicolumn{2}{|c|}{ short-term trigger $(\tau)$} & 2 & 8 & 6 & 0 & 0.08 & 1.67 & 3.33 & 15 & 0 & 0.151 & 1 & 0.911 & 9 & 0 & 0.08 & 0.328 \\
\hline \multicolumn{2}{|c|}{ short-term trigger $(\alpha)$} & 0.01 & 9 & 6 & 1 & 0.08 & 1.67 & 3.33 & 21 & 1 & 0.14 & 8 & 0.612 & 15 & 1 & 0.08 & 0.811 \\
\hline \multicolumn{2}{|c|}{ short-term trigger (term) } & 3 & 10 & 3 & 0 & 0.08 & 1.67 & 3.33 & 24 & 1 & 0.132 & 9 & 0.634 & 15 & 1 & 0.08 & 0.811 \\
\hline \multicolumn{2}{|c|}{ Incremental AMAs: } & $\rho$, monitoring & 11 & 1 & 0 & 0.08 & 1.67 & 3.33 & 25 & 1 & 0.126 & 9 & 0.656 & 10 & 1 & 0.08 & 0.811 \\
\hline \multicolumn{2}{|c|}{ step 1} & $0.8,2$ & 12 & 2 & 1 & 0.08 & 1.67 & 3.33 & 27 & 2 & 0.121 & 18 & 0.375 & 6 & 1 & 0.08 & 0.811 \\
\hline \multicolumn{2}{|l|}{ step 2} & $0.7,2$ & 13 & 6 & 2 & 0.08 & 1.67 & 3.33 & 33 & 4 & 0.117 & 35 & NA & 9 & 3 & 0.08 & 0.995 \\
\hline \multicolumn{2}{|c|}{ step 3} & 0,0 & 14 & 6 & 0 & 0.08 & 1 & 2 & 39 & 4 & 0.115 & 36 & NA & 14 & 3 & 0.08 & 0.995 \\
\hline Reversion tri & $\operatorname{ger}(\alpha)$ & 0.1 & 15 & 3 & 1 & 0.3 & 0.8 & 1.6 & 42 & 5 & 0.123 & 42 & 0.044 & 15 & 3 & 0.131 & 0.979 \\
\hline Reversion tri & $\operatorname{ger}(\rho)$ & 0.6 & 16 & 1 & 0 & 0.3 & 0.8 & 1.6 & 43 & 5 & 0.13 & 39 & 0.068 & 10 & 1 & 0.215 & 0.506 \\
\hline & & & 17 & 3 & 0 & 0.08 & 0.8 & 1.6 & 46 & 5 & 0.128 & 40 & 0.08 & 7 & 1 & 0.227 & 0.48 \\
\hline & & & 18 & 3 & 0 & 0.08 & 0.8 & 1.6 & 49 & 5 & 0.126 & 41 & 0.093 & 7 & 0 & 0.153 & 0.217 \\
\hline & & & 19 & 2 & 0 & 0.08 & 0.8 & 1.6 & 51 & 5 & 0.125 & 41 & 0.106 & 8 & 0 & 0.08 & 0.328 \\
\hline & & & 20 & 1 & 0 & 0.08 & 0.8 & 1.6 & 52 & 5 & 0.123 & 42 & 0.121 & 6 & 0 & 0.08 & 0.328 \\
\hline & & & 21 & 0 & 0 & 0.08 & 0.8 & 1.6 & 52 & 5 & 0.122 & 42 & 0.136 & 3 & 0 & 0.08 & 0.328 \\
\hline & & & 22 & 1 & 1 & 0.08 & 0.8 & 1.6 & 53 & 6 & 0.12 & 51 & 0.068 & 2 & 1 & 0.08 & 0.811 \\
\hline & & & 23 & 2 & 0 & 0.08 & 0.8 & 1.6 & 55 & 6 & 0.119 & 51 & 0.077 & 3 & 1 & 0.08 & 0.811 \\
\hline & & & 24 & 4 & 1 & 0.08 & 0.8 & 1.6 & 59 & 7 & 0.118 & 60 & 0.037 & 7 & 2 & 0.08 & 0.966 \\
\hline & & & 25 & 2 & 0 & 0.08 & 0.8 & 1.6 & 61 & 7 & 0.117 & 61 & NA & 8 & 1 & 0.08 & 0.811 \\
\hline & & & 26 & 0 & 0 & 0 & 0 & 0 & 61 & 7 & NA & NA & NA & NA & NA & NA & NA \\
\hline & & & 27 & 0 & 0 & 0 & 0 & 0 & 61 & 7 & NA & NA & NA & NA & $\mathrm{NA}$ & NA & NA \\
\hline & & & 28 & 0 & 0 & 0 & 0 & 0 & 61 & 7 & NA & NA & NA & NA & NA & NA & NA \\
\hline & & & 29 & 0 & 0 & 0 & 0 & 0 & 61 & 7 & NA & NA & NA & NA & NA & NA & NA \\
\hline & & & 30 & 0 & 0 & 0 & 0 & 0 & 61 & 7 & NA & NA & NA & NA & NA & NA & NA \\
\hline Edit/Close & $\begin{array}{l}\text { Save to file } \\
\text { (.txt) }\end{array}$ & $\begin{array}{l}\text { Generate } \\
\text { Another }\end{array}$ & & & & & & & & & & & & & & & \\
\hline
\end{tabular}

Figure 34. Screen capture of Scenario Explorer, one example (reversion trigger). No carcasses were found in the first 6 years of monitoring $(X=0)$, and evidence was strong enough to conclude that $\lambda<\tau \rho$ at $90 \%$ credibility (green cells), so the $\rho_{0}$ constraint was lifted. 


\subsubsection{Calculate}

The Calculate button gives a graphical summary of the triggering and mortality statistics for simulated projects.

\subsubsection{Example: Default parameters but with reversion trigger and incremental AMAs implemented}

Results for the scenario described in the previous section (fig. 31) but with 1000 simulation draws are given in figure 35. Fatalities are generated at a rate of $\lambda=2$ per year on average, so the average total number of fatalities in 30 years at sites without AMA would be 60 , with $M \leq 60$ at about half the sites and $M>60$ at about half the sites (fig. 29). However, the short- and long-term triggers initiate AMAs to reduce mortality rates when carcasses accumulate too rapidly, so in this scenario, fewer than 60 animals are killed on average. In the box-and-whisker plots, the horizontal central bars represent medians, and the bottoms and tops of the boxes show the 25 th and 75 th percentiles of the distribution.

The middle graph shows summary distributions of the number of years of operation before avoidance (that is, until the avoidance AMA is first implemented, either when the long-term trigger is fired or the incremental AMAs associated with the short-term trigger have advanced to the final step) or reversion. In the box-and-whisker plots, the horizontal central bars represent medians, and the bottoms and tops of the boxes show the 25th and 75th percentiles of the distribution. The median number of years before avoidance cannot be seen on the graph, which indicates that less than $50 \%$ of the projects were in avoidance by the end of the permit term. The bottom of the box is at 24 , indicating that $25 \%$ of projects reached avoidance AMA in the 24th year or earlier. The bottom of the box for reversion is not visible because fewer than $25 \%$ were ever in reversion during the permit term. The bottom of the solid whisker indicates that about $10 \%$ of projects the reversion trigger fired within 6 years.

The graph on the right shows the frequency of short-term trigger firings. The green bar shows that in nearly $80 \%$ of projects, the short-term trigger did not fire at all. At first glance, it may seem surprising that the short-term trigger fired so frequently. In a scenario with $\lambda=\tau$ (like in the example), $\alpha=0.01$ means that there is approximately a $99 \%$ chance the trigger would not fire in a single test. However, the short-term trigger is tested every year, and the probability that the trigger fires in at least one of the 30 years is $26 \%$ - much greater than the $1 \%$ probability that it fires in a given single year. In addition, the reversion trigger occasionally fires in the example, which increases the mortality rate in future years and thus increases the probability of the short-term trigger firing. The yellow bar indicates that in about $15 \%$ of projects the short-term trigger fired 1 time, and top of the red shows that in over $99 \%$ of projects, the short-term trigger fired fewer than 3 times.

\subsubsection{Example: Action of Long-Term Trigger and Frequency of Short-Term Triggering When $\lambda>\mathrm{T} / n$}

The long-term trigger is designed to signal when the number of fatalities has exceeded the permitted total or threshold, T. If avoidance AMA reduces future mortality rate to 0 after the long-term trigger has fired, then the action of the long-term trigger will not prevent the number of fatalities from exceeding T. However, typically the degree of exceedance will be minor, even when the baseline fatality rate far exceeds $\mathrm{T} / n$. In addition, in those cases, the short-term trigger gives early warning that the mortality rate may be higher than expected, which gives opportunity to make changes in operations or in permit terms to ensure that the number of fatalities remains in an acceptable range. 

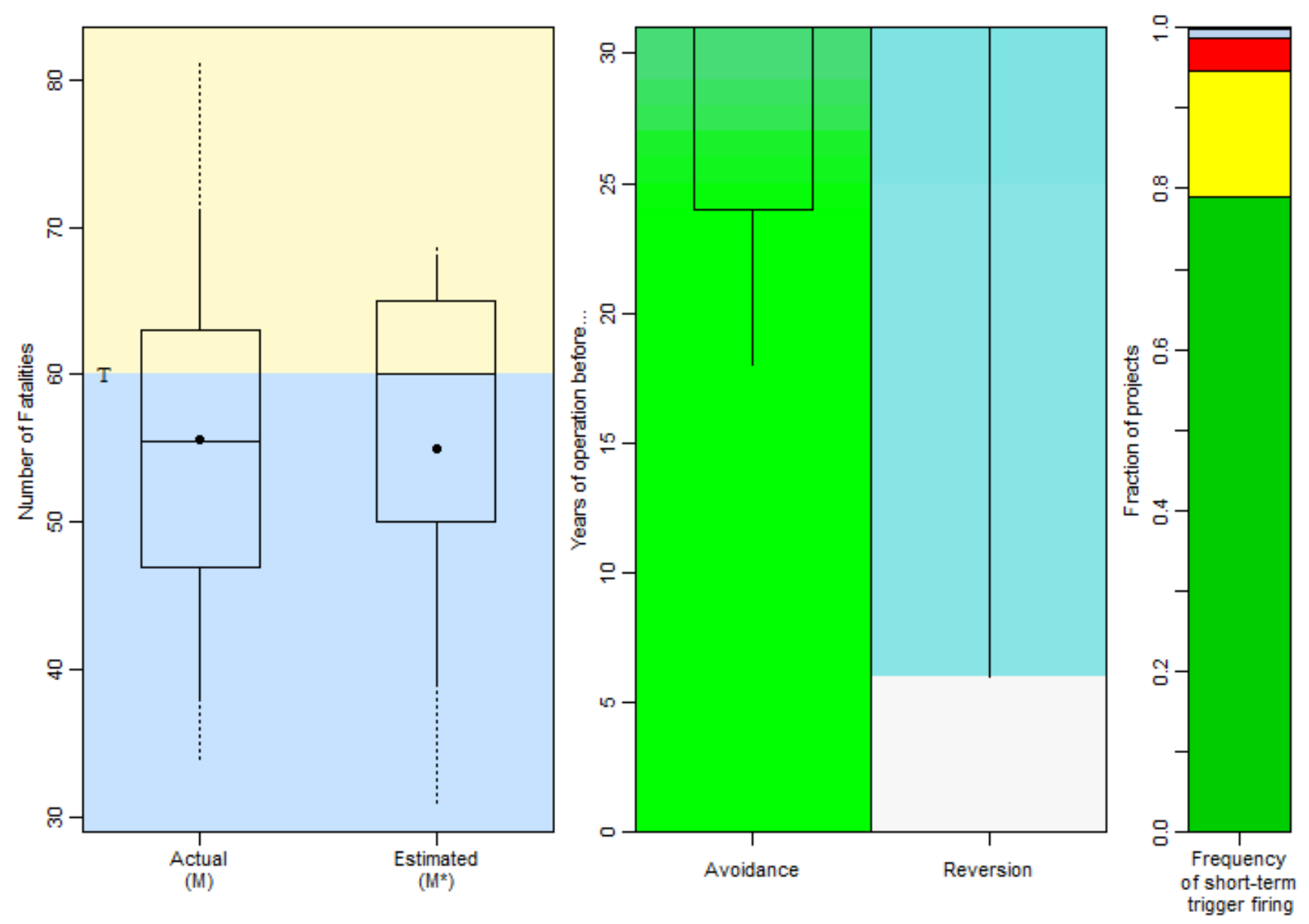

Take limit $(\mathrm{T})=60$ in $30 \mathrm{yrs}$, Baseline fatality rate $(\lambda)=2$, number of reps $=1000$

Monitoring: 3 years of intensive monitoring at start of permit

Detection probability, intensive monitoring: $\mathrm{g}=0.3$ with $95 \% \mathrm{CI}=[0.25,0.35]$

Detection probability, non-intensive monitoring: $\mathrm{g}=0.08$ with $95 \% \mathrm{CI}=[0.07,0.09]$

Long-term trigger: $\alpha=0.5$

Reversion trigger: $\rho 0=0.6, \alpha=0.1$

Short-term trigger: $\alpha=0.01$ for rolling average rate over 3 years, $\tau=2$

Incremental AMA: $\rho=0.8,0.7,0$

and 220 years of intensive monitoring following each trigger firing

Boxplots show IQR with median, and whiskers to show 5 th, 10 th, 90 th, and 95 th percentiles; $\bullet=$ mean

Number of short-term trigger firings in project:

\begin{tabular}{|l|l|l|l|l|}
\hline 0 & 1 & 2 & 3 & $4+$ \\
\hline
\end{tabular}

Figure 35. Box and whisker plots generated by Evidence of Absence software showing Scenario Explorer results for default parameters but with active reversion trigger, incremental AMA, and 1000 simulation draws. 
The actions of the short- and long-term triggers in a scenario where the baseline fatality rate is 3 times the permitted annual rate of $\mathrm{T} / n$ are explored using the Scenario Explorer module. To begin, select "Restore defaults" from the Edit menu to load the default parameter set. Set the baseline fatality rate to 6 and the number of simulation draws to 10000 (fig. 36). If the fatality rate is $6 / \mathrm{yr}$, on average there will be 180 fatalities over the course of 30 years, which is 3 times the permitted rate of $\mathrm{T}=60$ in this example. Also, since the scenario does not include the reversion trigger, incremental AMAs, or intensive monitoring following the long-term trigger, the calculations are relatively fast and 10,000 simulation draws can be calculated quickly. The short-term trigger is activated, but it is not associated with incremental AMAs. In this case, the simulation tallies the number of times the short-term trigger fires in each project, but the triggering does not induce AMAs or affect fatality rates or monitoring protocols.

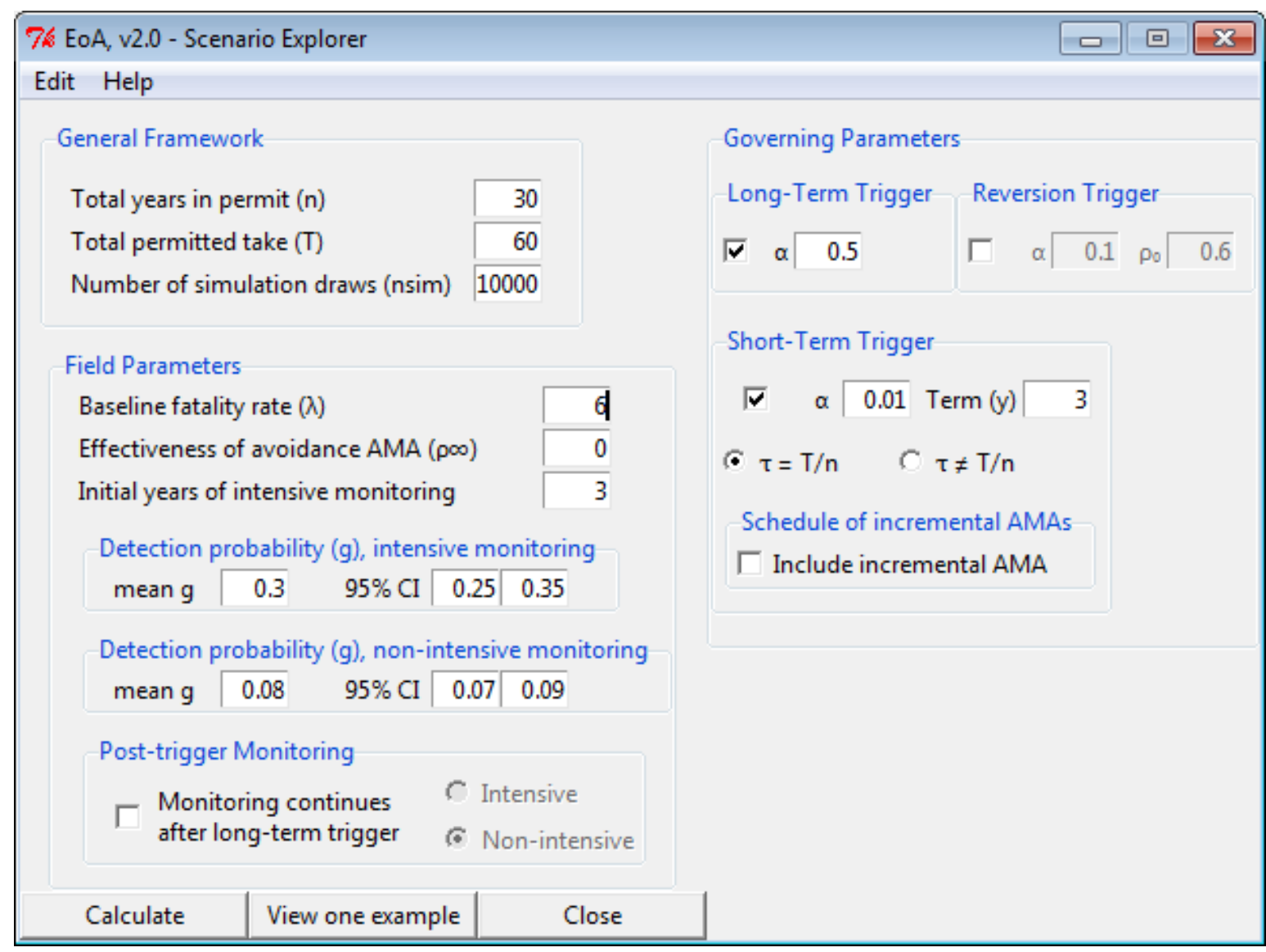

Figure 36. Screen capture of parameters for exploring the action of short- and long-term triggers in a scenario with $\lambda=3 \mathrm{~T} / n$.

The results show that the avoidance AMA implemented after the long-term trigger fired kept the number of fatalities far below the 180 that would be expected under unconstrained conditions (fig. 37, left panel). The average number of fatalities $(M)$ was slightly higher than the permitted 60 because the long-term trigger is designed to fire when $M>60$ and not before. In particular, the average number of fatalities was approximately $T+\lambda$, which is a result of fatalities accumulating at a rate of $\lambda / \mathrm{yr}$ until the total exceeds T. The estimated number of fatalities $\left(M^{*}\right)$ at the time of triggering is much less variable 
than the actual number of fatalities $(M)$. That is because $M^{*}$ cannot be less than $\mathrm{T}$ at triggering (by definition) and cannot be substantially greater than $\mathrm{T}$ in this scenario $\left(\rho_{\infty}=0\right)$ because fatalities stop accumulating after $M^{*}>\mathrm{T}$. The median number of years before avoidance (that is, before the long-term trigger fired) was 10 (fig. 37, middle panel), which is how long it would take for the expected number of fatalities to reach 60 when the average annual rate is $\lambda=6$. The short-term trigger fired in about $84 \%$ of the projects (fig. 37, right panel) and fired 3 or more times in about half the projects.
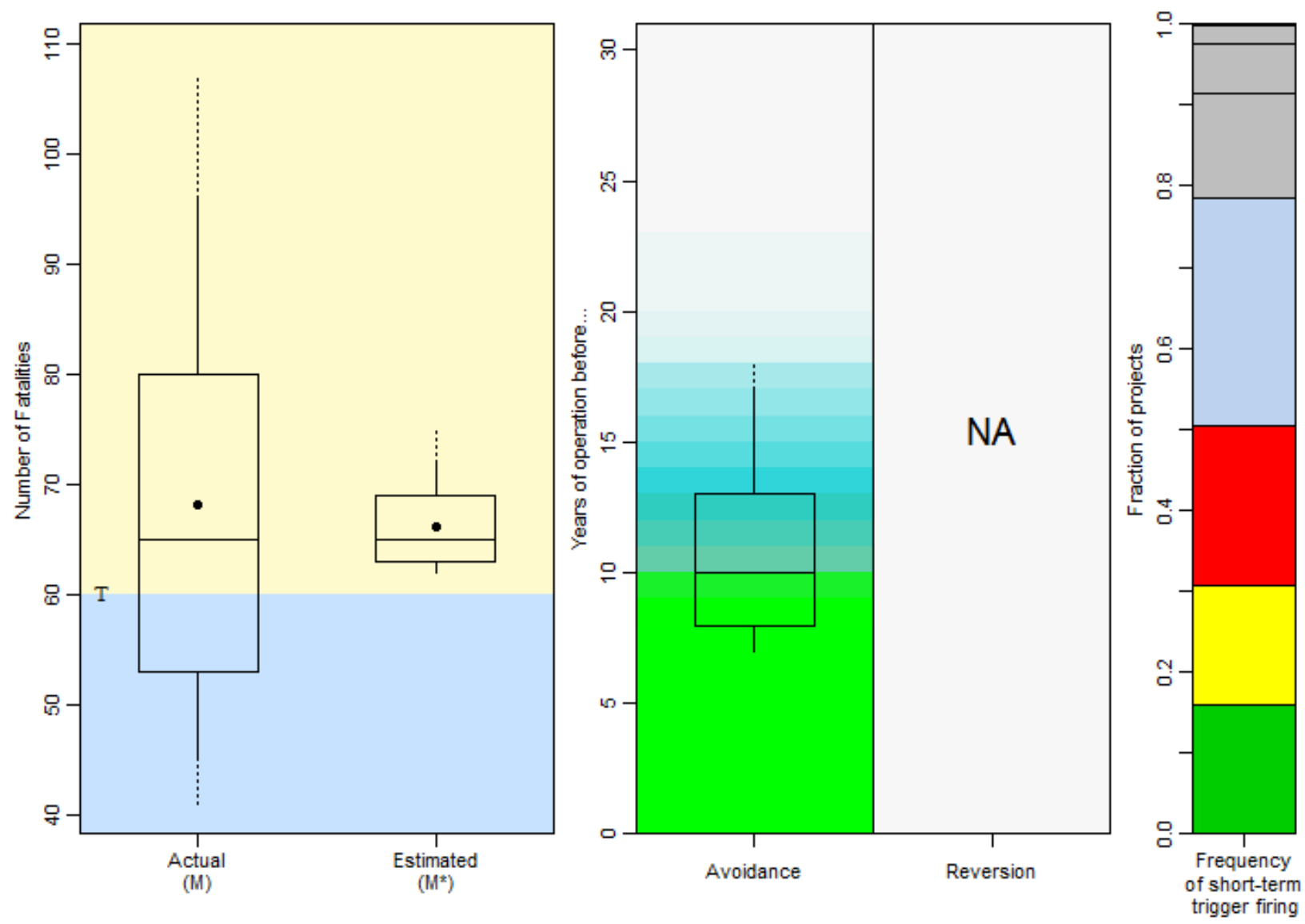

Take limit $(T)=60$ in $30 \mathrm{yrs}$, Baseline fatality rate $(\lambda)=6$, number of reps $=10000$

Monitoring: 3 years of intensive monitoring at start of permit

Detection probability, intensive monitoring: $\mathrm{g}=0.3$ with $95 \% \mathrm{CI}=[0.25,0.35]$

Detection probability, non-intensive monitoring: $\mathrm{g}=0.08$ with $95 \% \mathrm{CI}=[0.07,0.09]$

Long-term trigger: $\alpha=0.5$

Reversion trigger: NA

Short-term trigger: $\alpha=0.01$ for rolling average rate over 3 years, $\tau=2$

with no intensive monitoring after triggering

Boxplots show IQR with median, and whiskers to show 5 th, 10 th, 90 th, and 95 th percentiles; $\bullet=$ mean Number of short-term trigger firings in project:

\begin{tabular}{l|l|l|l|l|}
0 & 1 & 2 & 3 & $4+$
\end{tabular}

Figure 37. Box and whisker plots generated by Evidence of Absence software showing Scenario Explorer. Action of long-term trigger and frequency of short-term triggering when $\lambda=3 \mathrm{~T} / n$. 


\subsubsection{Example: Action of Long-Term Trigger and Frequency of Short-Term Triggering When $\lambda<\mathrm{T} / n$}

When $\lambda<\mathrm{T} / n$ the long-term trigger does not often fire because the total number of fatalities will usually be less than T. If $\lambda$ is changed from 6 to 1 in the scenario described in the previous example (fig. 36), then the expected total number of fatalities over the course of the project would be 30 , which is substantially smaller than the permitted 60 . In 10,000 simulated projects under this scenario, the longterm trigger never fired. The short-term trigger fired in about $3 \%$ of the projects, but almost never fired more than once (fig. 38).
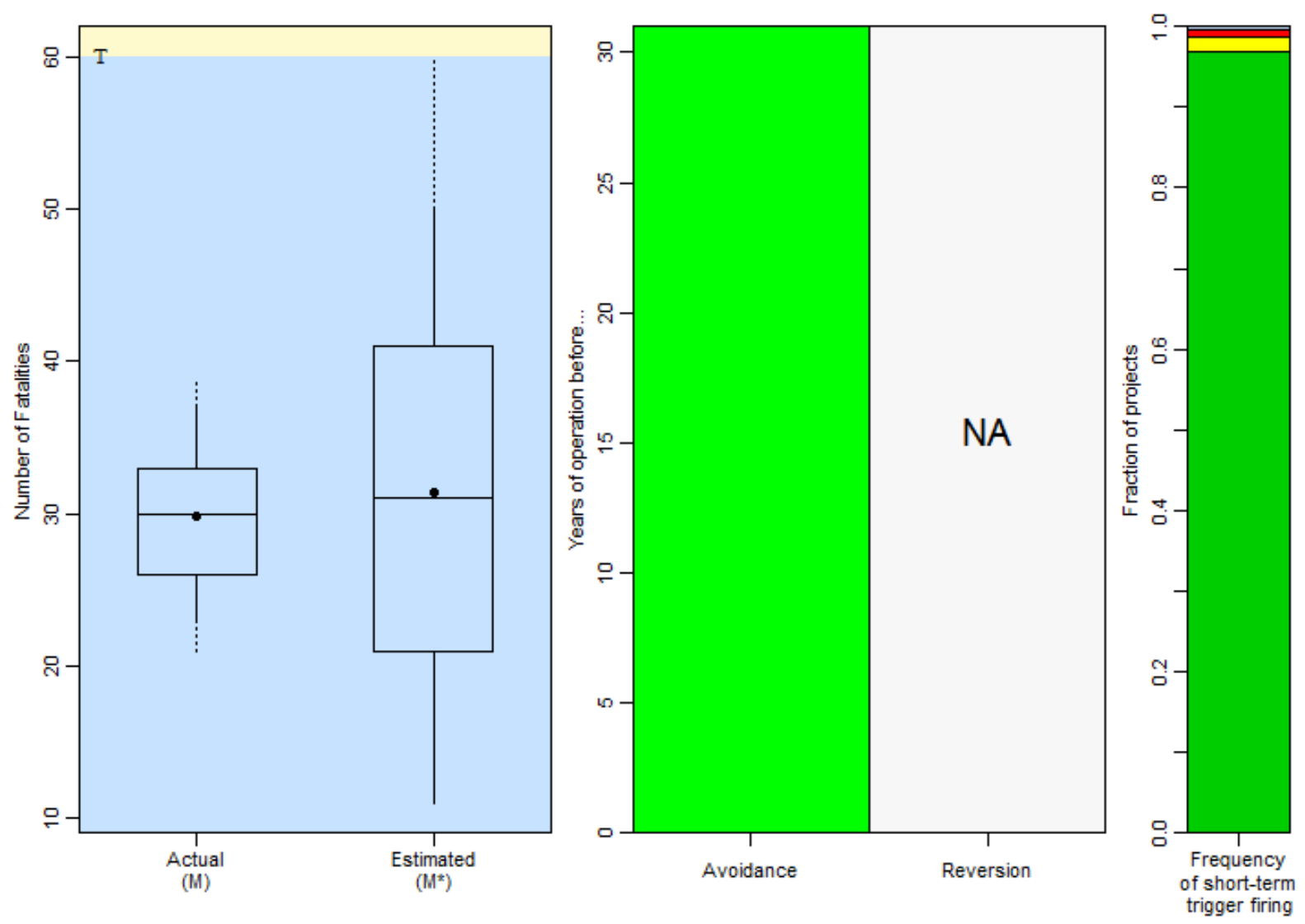

Take limit $(T)=60$ in 30 yrs, Baseline fatality rate $(\lambda)=1$, number of reps $=10000$

Monitoring: 3 years of intensive monitoring at start of permit

Detection probability, intensive monitoring: $\mathrm{g}=0.3$ with $95 \% \mathrm{CI}=[0.25,0.35]$

Detection probability, non-intensive monitoring: $g=0.08$ with $95 \% \mathrm{CI}=[0.07,0.09]$

Long-term trigger: $\alpha=0.5$

Reversion trigger: NA

Short-term trigger: $\alpha=0.01$ for rolling average rate over 3 years, $\tau=2$

with no intensive monitoring after triggering

Boxplots show IQR with median, and whiskers to show 5 th, 10 th, 90 th, and 95 th percentiles; $\bullet=$ mean

Number of short-term trigger firings in project:

\begin{tabular}{|l|l|l|l|l|}
\hline 0 & 1 & 2 & 3 & $4+$ \\
\hline
\end{tabular}

Figure 38. Box and whisker plots generated by Evidence of Absence software showing Scenario Explorer. Action of long-term trigger and frequency of short-term triggering when $\lambda=0.5 \mathrm{~T} / n$. 


\section{Acknowledgments}

This project was inspired by feedback from users of EoA v1.0, who not only provided the initial motivation but also continual suggestions, corrections, questions, and insights as the project progressed. TJ Miller (FWS, retired) made an enormous effort to understand the statistics and was a constant source of inspiration, support, and insight in the early stages of the project. Jim Hines (USGS) gave substantial, valuable instruction and support for tcltk, which made it possible to design the slick user interface and break free of the clunky dependence on Excel ${ }^{\circledR}$ which was a common complaint with EoA v1.0. Mitch Craig (SunEdison, Inc.) patiently and persistently rooted out many bugs, and, over the course of a year or more, asked probing questions and made suggestions that resulted in a much better product. Tom Snetsinger (Tetra Tech, Inc.) similarly provided long-running support in the form of questions and suggestions. Other users made concrete suggestions or raised questions that led to immediate improvements in the product: Greg Aldrich (Duke Energy Environmental Services), Richard Borthwick (Copperhead Environmental Consulting, Inc.), Julie Garvin (Tetra Tech, Inc), John Guarnaccia (Guarnaccia Ecological Services), Fränzi Korner-Nievergelt (oikostat GmbH), Kathleen MacGregor (Ministère des Forêts, de la Faune et des Parcs, Quebec), Joana Marques (Bioinsight), Peter Owens (Arcus Consultancy Services, Ltd), Alex Prichard (ABR, Inc.), Paul Rabie (Western Ecosystems Technology, Inc.), Amber Schorg (FWS), and Diane Sether (FWS). Leslie New (Washington State University), Matt Stuber (FWS), and Emily Bjerre (FWS) provided inspiration and guidance for thinking about special issues involved in estimating take of eagles. Western Pacific Regional Fishery Management Council and the National Marine Fisheries Service raised a number of new questions about the potential use of EoA in estimating incidental bycatch of sea turtles and whales in the Hawaii longline tuna fisheries. We made a number of improvements to the software to address some of these questions - special thanks to Asuka Ishizaki (NOAA), Marti McCracken (NOAA), Milani Chalupka (Western Pacific Regional Fishery Management Council), and Craig Johnson (HDR, Inc.). Lance Roy (homeschool Corvallis) and Mark Dalthorp (Crescent Valley High School) helped with the statistical modeling and with optimizing computer code. With 100 percent certainty, many others have contributed, and we are grateful for their help and apologize for failing to include them in the list. Even with the attention and help of so many capable people, there are still bound to be imperfections and errors, which we are happy to take full credit for. 


\section{References Cited}

Arnett, E.B., 2006, A preliminary evaluation on the use of dogs to recover bat fatalities at wind energy facilities: Wildlife Society Bulletin, v. 34, no. 5, p. 1440-1445.

Arnett, E.B., Huso, M.M.P., Schirmacher, M.R., and Hayes, J.P., 2011, Altering turbine speed reduces bat mortality at wind-energy facilities: Frontiers in Ecology and the Environment, v. 9, no. 4, 209 214, doi:10.1890/100103.

Arnett, E.B., Hein, C.D., Schirmacher, M.R., Huso, M.M.P., Szewczak, J.M., 2013, Evaluating the effectiveness of an ultrasonic acoustic deterrent for reducing bat fatalities at wind turbines:PLoS ONE, v. 8, no. 6, e65794, doi:10.1371/journal.pone.0065794.

Behr, O., Brinkmann, R., Korner-Nievergelt, F., Nagy, M., Niermann, I., Reich, M., and Simon, R., 2016, Reducing the collision risk for bats at onshore wind turbines (RENEBAT II): Report by Friedrich Alexander Universität Erlangen Nürnberg and Leibniz Universität Hannover, 374 p., accessed November 15, 2016, at https://tethys.pnnl.gov/publications/reducing-collision-risk-batsonshore-wind-turbines-renebat-ii.

Bengtsson, H., 2016, matrixStats-Functions that apply to rows and columns of matrices (and to Vectors): The Comprehensive R Archive Network Web page, R package version 0.51.0., https:/CRAN.R-project.org/package $=$ matrixStats

Berger, J.O., Bernardo, J.M., and Sun, D., 2012, Objective priors for discrete parameter spaces: Journal of the American Statistical Association: v. 107, no. 498, p. 636-648, DOI:10.1080/01621459.2012.682538

Bispo, R., Bernardino, J., Marques, T.A., and Pestana, D., 2012, Modeling carcass removal time for avian mortality assessment in wind farms using survival analysis: Environmental and Ecological Statistics, v. 20, no. 1, p. 147-165, doi:10.1007/s10651-012-0212-5.

Boogaart, K.G. van den, 2010, tensorA-Advanced tensors arithmetic with named indices: The Comprehensive R Archive Network Web page, R package version 0.36, https:/CRAN.Rproject.org/package=tensorA.

Bulling, L., and Köppel, J., 2016, Exploring the trade-offs between wind energy and biodiversity conservation, in Geneletti, Davide, ed., Handbook on biodiversity and ecosystem services in impact assessment: Northampton, Massachusetts, Edward Elgar Publishing, p. 299-330.

Burnham, K.P. and Anderson, D.R., 2004, Multimodel inference: understanding AIC and BIC in model selection: Sociological Methods \& Research, v. 33, p. 261-304.

Chang, W., 2016, R6-Classes with reference semantics: The Comprehensive R Archive Network Web page, R package version 2.2.0., https://CRAN.R-project.org/package=R6.

Dalthorp, D., Huso, Manuela, Dail, D., and Kenyon, J., 2014, Evidence of absence software user guide: U.S. Geological Survey Data Series 881, 34 p., http://dx.doi.org/10.3133/ds881.

Dalthorp, D., and Huso, M.M.P., 2015, A framework for decision points to trigger adaptive management actions in long-term incidental take permits: U.S. Geological Survey Open-File Report 2015-1227, 88 p., http://dx.doi.org/10.3133/ofr20151227.

Dutang, C., Goulet, V., and Pigeon, M., 2008, actuar-An R Package for Actuarial Science: Journal of Statistical Software, v. 25, no. 7, 1-37.

Grosjean, P., 2016, SciViews-A GUI API for R. UMONS: Mons, Belgium, SciViews Web page, http://www.sciviews.org/SciViews-R.

Hankin, R.K.S., 2006, Special functions in R-Introducing the gsl package: R News, v. 6, no. 4. Horvitz, D.G., and Thompson, D.J., 1952, A generalization of sampling without replacement from a finite universe: Journal of the American Statistical Association, v. 47, p. 663-685. 
Huso, M.M.P., 2011, An estimator of wildlife fatality from observed carcasses: Environmetrics, v. 22 , no. 3, p. 318-329, doi:10.1002/env.1052.

Huso, M.M.P., Som, N., Ladd, L., 2012, Fatality estimator user's guide: U.S. Geological Survey Data Series 729, 22 p., https://dx.doi.org/10.3133/ds729.

Huso, M.M.P., and Dalthorp, D., 2014, Accounting for unsearched areas in estimating wind turbinecaused fatality: Journal of Wildlife Management, v. 78, p. 347-358, doi: 10.1002/jwmg.663.

Huso, M.M.P., Dalthorp, D., Dail, D., and Madsen, L., 2015, Estimating wind-turbine-caused bird and bat fatality when zero carcasses are observed: Ecological Applications, v. 25, p. 1,213-1,225.

Jeffreys, H., 1946, An invariant form for the prior probability in estimation problems: Proceedings of the Royal Society of London, Series A, Mathematical and Physical Sciences, v. 186, no. 1007, p. 453-461.

Kaplan, E.L., and Meier, P., 1958, Nonparametric estimation from incomplete observations: Journal of the American Statistical Association, v. 53, no. 282, p. 457-481.

Korner-Nievergelt, F., Behr, O., Brinkmann, R., Etterson, M.A., Huso, M.M.P., Dalthorp, D., KornerNievergelt, P., Roth, T., and Niermann, I., 2015, Mortality estimation from carcass searches using the R-package carcass - A tutorial: Wildlife Biology, v. 21, no. 1, p. 30-43.

Korner-Nievergelt, F., Korner-Nievergelt, P., Behr, O., Niermann, I., Brinkmann, R., and Hellriegel, B., 2011, A new method to determine bird and bat fatality at wind energy turbines from carcass searches: Wildlife Biology, v. 17, p. 350-363.

Marten Law, 2015, Golden eagle mortality at wind energy projects-Implications of Duke and PacifiCorp plea agreements: Marten Law Web page, accessed November 15, 2016, at http://www.martenlaw.com/newsletter/20150121-golden-eagle-mortality-wind-energy-projects.

Mathews, F., Swindells, M., Goodhead, R., August, T.A., Hardman, P., Linton, D. M., and Hosken, D.J. 2013, Effectiveness of search dogs compared with human observers in locating bat carcasses at windturbine sites - A blinded randomized trial: Wildlife Society Bulletin, v. 37, no. 1, p. 34-40. doi:10.1002/wsb.256.

Paula, J., Costa Leal, M., Silva, M.J., Mascarenhas, R., Costa, H., Mascarenhas, M., 2011, Dogs as a tool to improve bird-strike mortality estimates at wind farms: Journal for Nature Conservation, v. 19 (2011), 202-208, doi:10.1016/j.jnc.2011.01.002.

Plummer, M., 2003, JAGS - A program for analysis of Bayesian graphical models using Gibbs sampling: CiteSeer ${ }^{\mathrm{x}}$, doi:10.1.1.13.3406.

Plummer, M., 2016, rjags-Bayesian Graphical Models using MCMC: The Comprehensive R Archive Network Web page, R package version 4-6, https://CRAN.R-project.org/package=rjags.

R Core Team, 2016, R: A language and environment for statistical computing: R Foundation for Statistical Computing, Vienna, Austria, https://www.R-project.org/.

Shoenfeld, P., 2004, Suggestions regarding avian mortality estimation: Mountaineer Wind Energy Center Technical Review Committee; Report for the West Virginia Highlands Conservancy, West Virginia, $6 \mathrm{p}$.

Sinclair, K., and DeGeorge, E., 2016, Wind energy industry eagle detection and deterrents: Research Gaps and Solutions Workshop Summary Report, Report by National Renewable Energy Laboratory (NREL). 49 p.

Therneau, T., 2015, A Package for Survival Analysis in S: The Comprehensive R Archive Network Web page, version 2.38, http://CRAN.R-project.org/package=survival.

Therneau, T.M., and Grambsch, P.M., 2000, Modeling survival data-Extending the Cox Model: New York, Springer, 350 p., ISBN 0-387-98784-3. 
Tierney, L., 2011, tkrplot-TK Rplot: The Comprehensive R Archive Network Web page, R package version 0.0-23, https://CRAN.R-project.org/package=tkrplot.

Venables, W.N., and Ripley, B.D., 2002, Modern applied statistics with S (4th ed.): Springer, New York, ISBN 0-387-95457-0, 498 p.

Warren-Hicks, W., Newman, J., Wolpert, R., Karas, B., and Tran, L., eds., 2013, Improving methods for estimating fatality of birds and bats at wind energy facilities: CalWEA, California Energy Commission, CEC-500-2012-086, Berkeley, California, 136 p.

Wolpert, R.L., 2012, Appendix A-A new equation for estimating avian mortality rates - A partially periodic equation for estimating avian mortality rates, in Warren-Hicks, W., Newman, J., Wolpert, R., Karas, B., and Tran, L., eds., 2013, Improving methods for estimating fatality of birds and bats at wind energy facilities: CalWEA, California Energy Commission, publication CEC-500-2012-086, p. A-1-A-20.

Wolpert, R.L., 2015, ACME-A partially periodic estimator of avian \& chiropteran mortality at wind turbines: Cornell University Library Web page, http://arxiv.org/abs/1507.00749.

Wolpert, R.L., and Coleman, J., 2015, acmeR-Implements ACME estimator of bird and bat mortality by wind turbines: The Comprehensive R Archive Network Web page, R package version 1.1.0, https://CRAN.R-project.org/package=acmeR.

Yee, T.W., 2017, VGAM-Vector generalized linear and additive models: The Comprehensive R Archive Network Web page, R package version 1.0-3, https:/CRAN.R-project.org/package=VGAM. 


\section{Appendix A. Estimator of Overall Detection Probability $(g)$-Single Year, Single Class}

For a single search class within a single year, the EoA model estimates the overall detection probability for carcasses arriving in the searched area during the monitoring season. The season is partitioned into $n$ search intervals, $I_{i}$ for $i=1, \ldots, n$ with $n+1$ searches conducted at times $t=$ $0, t_{1}, \ldots, t_{n}$. The monitoring season is assumed to begin at time $t=0$, and all carcasses found in searches at $t_{1}, \ldots, t_{n}$ are assumed to have arrived after time $t=0$. This condition is commonly met in practice by either beginning the monitoring before fresh carcasses begin to arrive in the spring, or by conducting a thorough "clean-out" search at time $t=0$ and not including the found carcasses in the final total. The basic estimator equation for detection of carcasses that arrived in the search area during the monitored period is:

$$
\hat{g}=\sum_{i=1}^{n} \sum_{j=i}^{n} k^{j-i} p\left[\prod_{s 0}^{j-i-1}\left(1-I_{\{s \geq 0\}} k^{s} p\right)\right] \int_{t_{i-1}}^{t_{i}} S\left(t_{j}-t\right) v(t) d t
$$

where:

$\hat{g}$ detection probability for carcasses arriving in searched area during monitored period;

$p=$ searcher efficiency on the first search after carcass arrival;

$k=$ the fractional change in searcher efficiency with each successive search

$$
I_{\{s \geq 0\}}=\left\{\begin{array}{l}
1 \text { if } s \geq 0 \\
0 \quad \text { otherwise }
\end{array} ;\right.
$$

$S=$ persistence distribution;

$v=$ arrival function;

$i, j=$ search interval for carcass arrival and search, respectively; and

$s=$ number of times a carcass has been missed in previous searches.

This generalized model can accommodate a number of realistic scenarios that many commonly used estimators cannot, for example, a scavenging rate that varies with carcass age, a decrease in searcher efficiency for carcasses that have been missed in previous searches, and an irregular search schedule (appendix K). 
The persistence distribution, $S(t)$, gives the proportion of carcasses persisting $t$ units (usually days) after arrival. In the software, the user can choose among exponential, lognormal, log-logistic, and Weibull distributions. The arrival function, $v(t)$, gives the relative arrival rate for carcasses through the monitoring period, and $v$ defines a PDF of arrival times so that $\int_{0}^{t_{n}} v(t) d t=1$. In the software, a constant arrival function is assumed, but advanced users may use EoA's R command line tools to enter a custom arrival function in some situations (appendix I).

To derive the estimator equation, let $C$ be the event of observing a carcass that arrives in the monitored area during the monitoring period. Let $g$ be the probability of $C$, or $g=P(C)$, which is calculated by conditioning on time of carcass arrival. Let $A_{i}$ be the event that the carcass arrives in interval $i$, so that $P(C)=\sum P\left(C \mid A_{i}\right) P\left(A_{i}\right)$. The components, $P\left(C \mid A_{i}\right)$, are calculated via partitioning the subsequent carcass searches. Define $C_{j}$ as the event that the carcass is observed in search $j$. Since carcasses are removed after being discovered, $C_{j}$ and $C_{h}$ are mutually exclusive if $j \neq h$. Also, $P\left(C_{j} \mid A_{i}\right)=0$ if $j<i$ because carcasses must arrive before they can be observed. Thus, $P\left(C \mid A_{i}\right)=$ $\sum_{j=i}^{n} P\left(C_{j} \mid A_{i}\right)$. Each term in the sum can be calculated as the product of:

1) $r_{i j}=$ the probability that a carcass that arrives in interval $i$ persists until search $j$, and

2) $p_{i j}=$ the probability that the carcass is not detected in searches $i, i+1, \ldots, j-1$ and is detected in search $j$.

The first probability is $r_{i j}=\int P\left(\right.$ persist until $t_{j}$ |arrive at $\left.t\right) P($ arrive at $t$ ), where the integral is calculated over the arrival interval. Thus:

$$
r_{i j}=\int_{t_{i-1}}^{t_{i}} S\left(t_{j}-t\right) \frac{v(t)}{\int_{t_{i-1}}^{t_{i}} v(t) d t} d t
$$

The second probability is $p_{i j}=P\left(\right.$ observe at $t_{j}$ but not observe at $\left.t_{s}<t_{j}\right)=(1-p)(1-$

$$
\begin{aligned}
& \text { kp) } \ldots\left(1-k^{j-i-1} p\right) k^{j-i} p \text {, so: } \\
& \quad p_{i j}=k^{j-i} p \prod_{s=0}^{j-i-1}\left(1-I_{\{s \geq 0\}} k^{s} p\right) \text { for } j>i, p_{i j}=p \text { for } j=i \text {, and } p_{i j}=0 \text { for } j<i .
\end{aligned}
$$

Therefore, the probability of observing in search $j$ a carcass that arrived in interval $i$ is:

$$
P\left(C_{j} \mid A_{i}\right)=k^{j-i} p\left[\prod_{s=0}^{j-i-1}\left(1-I_{\{s \geq 0\}} k^{s} p\right)\right] \cdot \int_{t_{i-1}}^{t_{i}} S\left(t_{j}-t\right) \frac{v(t)}{\int_{t_{i-1}}^{t_{i}} v(t) d t} d t
$$


Summing over all arrival times and search intervals, we get:

$$
\begin{gathered}
P(C)=\sum_{i=1}^{n} P\left(C \mid A_{i}\right) P\left(A_{i}\right)=\sum_{i=1}^{n} \sum_{j=i}^{n} P\left(C_{j} \mid A_{i}\right) P\left(A_{i}\right) \\
=\sum_{i} \sum_{j} k^{j-i} p\left[\prod_{s=0}^{j-i-1}\left(1-I_{\{s \geq 0\}} k^{s} p\right)\right] \int_{t_{i-1}}^{t_{i}} S\left(t_{j}-t\right) \frac{v(t)}{\int_{t_{i-1}}^{t_{i}} v(t) d t} d t \int_{t_{i-1}}^{t_{i}} v(t) d t \\
=\sum_{i=1}^{n} \sum_{j=i}^{n} k^{j-i} p\left[\prod_{s=0}^{j-i-1}\left(1-I_{\{s \geq 0\}} k^{s} p\right)\right] \int_{t_{i-1}}^{t_{i}} S\left(t_{j}-t\right) v(t) d t .
\end{gathered}
$$

If the fraction of carcasses arriving in the searched area is $a$, then the detection probability for carcasses arriving at the site during the monitored period would be $a \cdot \hat{g}$. Finally, if $v$ is the fraction of carcasses arriving during the monitored period, then the overall detection probability extrapolated to the entire site for the period of inference would be $v a \hat{g}$.

Uncertainty in $\hat{g}$ is accounted for by parametric bootstrapping of the persistence (appendix C) and searcher efficiency (appendix D) models to simulate $\hat{g}$ and using fitdistr from R package MASS (Venables and Ripley, 2002) to find the maximum likelihood estimates of the $B a$ and $B b$ parameters of a beta distribution fitting the simulated $\hat{g}$ 's. The number of simulation draws $(n)$ is a function of the estimated variance of $\hat{g}$, with more draws required when the uncertainty in $\hat{g}$ is greater. More precisely, $n=\min \left[\left(\frac{\hat{s}}{0.003 g_{0}}\right)^{2}, 20,000\right]$, where $\hat{s}$ is the estimated standard deviation of $\hat{g}$ based on a preliminary simulation of 1000 draws and $g_{0}$ is the overall detection probability for the maximum likelihood estimates of the input parameters. A $100(1-\alpha) \%$ CI for $g$ is defined as $\left[g_{l w r}, g_{u p r}\right]$ where $P(\hat{g} \leq$ $\left.g_{l w r}\right)=P\left(\hat{g} \geq g_{u p r}\right)=\alpha / 2$. 


\section{Appendix B. Calculation of the Posterior Distributions of $M \mid(X, \widehat{g})$ and $\lambda \mid(X, \widehat{g})$}

A posterior distribution of the total mortality $(M)$ given the carcass count $(X)$ and estimated detection probability $(\hat{g})$ is estimated as $P(M \mid X, \hat{g})=\frac{P(X \mid M, \hat{g}) P(M=m)}{\sum_{m} P(X \mid M=m, \hat{g}) P(M=m)}$, where $X \sim \operatorname{betabinomial}(M, B a, B b), B a$ and $B b$ are the estimated beta parameters characterizing the distribution of $\hat{g}$, and the prior distribution of $M$ is $P(M=m) \propto \sqrt{m+1}-\sqrt{m}$, which is the integrated reference prior for a binomial index (Berger and others, 2012). The prior is truncated at the smallest value $m$ such that $P(X \leq x \mid M=m)<0.0001$, where $x$ is the observed carcass count and $X \sim \operatorname{betabinomial}(m, B a, B b)$. In other words, the prior is set to zero for values of $m$ that are large enough to be practically incompatible with the search data.

Important summary statistics from the posterior distribution of $M$ include:

- $M^{*}$, the upper bound of a one-sided $100(1-\alpha) \%$ CI for $M$, or, $M^{*}=\min \{m \in \mathbb{Z}: P(M \leq$ $m) \geq 1-\alpha\}$

- a two-sided $100(1-\alpha) \%$ CI for $M$, defined as $\left[M_{l w r}, M_{u p r}\right]$, where $M_{l w r}$ is the greatest integer such that $P\left(M<M_{l w r}\right) \leq \alpha / 2$ and $M_{u p r}$ is the smallest integer such that $P\left(M \geq M_{l w r} \cap M \leq\right.$ $\left.M_{u p r}\right) \geq 1-\alpha$.

- the median of the posterior of $M$, which can be used as a point estimate of $M$

A posterior distribution of the fatality rate $(\lambda)$ given the carcass count $(X)$ and estimated detection probability $(\hat{g})$ is estimated as $P(\lambda \mid X, \hat{g})=\frac{P(X \mid \lambda, \hat{g}) P(\lambda)}{\int P(X \mid \lambda, \hat{g}) P(\lambda) d \lambda}$, where $X \sim \operatorname{binomial}(M, \hat{g})$, $M \mid(\lambda) \sim \operatorname{Poisson}(\lambda), \hat{g} \sim \operatorname{beta}(B a, B b), B a$ and $B b$ are the parameters characterizing the distribution of $\hat{g}$ (as described in appendix A), and $P(\lambda) \propto 1 / \sqrt{\lambda}$ is the non-informative Jeffreys prior (Jeffreys, 1946) for the rate parameter of a Poisson distribution. 


\section{Appendix C. Estimation of Carcass Persistence Parameters: $\alpha$ and $\beta$}

The four carcass persistence models (exponential, Weibull, log-logistic, and lognormal) are fit using the R package survival (Therneau and Grambsch, 2000; Therneau, 2015). In the simulation of $\hat{g}$, random parameter values for the persistence distribution are drawn from the fitted persistence model, which gives the estimated marginal means and variance-covariance matrix of the joint distribution of the persistence parameters. Let $\beta_{0}$ and $\beta_{1}$ be the intercept and scale parameters from the persistence model as parameterized in the survival package. Then, in the case of Weibull, log-logistic, or lognormal persistence models, simulated values of $\left(\beta_{0}, \beta_{1}\right)$ are drawn from a multivariate normal distribution with mean of $\boldsymbol{\mu}=\left(\hat{\beta}_{0}, \hat{\beta}_{1}\right)$ and variance $\boldsymbol{\Sigma}=\widehat{\Sigma}\left(\hat{\beta}_{0}, \hat{\beta}_{1}\right)$ as given in the results of the survival fitted model. In the case of an exponential persistence model, only one parameter $\left(\beta_{0}\right)$ is estimated, and simulated values are drawn from a normal distribution with mean $\mu=\hat{\beta}_{0}$ and $\sigma=\sqrt{\hat{\sigma}_{\widehat{\beta}_{0}}^{2}}$. EoA's parameterizations differ from those used by the survival package, and the simulated $\beta_{0}$ and $\beta_{1}$ values are converted to EoA's $\alpha$ and $\beta$ before plugging them into the estimator equation for simulation of $\hat{g}$.

Table C1. Converting parameters from survival package to EoA persistence parameters.

[The $\beta_{0}$ and $\beta_{1}$ parameters referred to in the table correspond the intercept and scale parameters in the survival package]

\begin{tabular}{|l|c|c|}
\hline \multicolumn{1}{|c|}{ Distribution } & $\boldsymbol{\alpha}$ & $\boldsymbol{\beta}$ \\
\hline Exponential & - & $e^{\beta_{0}}$ \\
\hline Weibull & $1 / \beta_{1}$ & $e^{\beta_{0}}$ \\
\hline Log-logistic & $1 / \beta_{1}$ & $e^{\beta_{0}}$ \\
\hline Lognormal & $\beta_{1}^{2}$ & $\beta_{0}$ \\
\hline
\end{tabular}




\section{Appendix D. Estimation of Searcher Efficiency Parameters: $\boldsymbol{p}$ and $\boldsymbol{k}$}

Let $p$ be the initial searcher efficiency for fresh carcasses, or, more precisely, the conditional probability of detecting a carcass on the first search after carcass arrived, given that the carcass is present at the time of the search. Let $k$ be the fractional change in searcher efficiency with each successive search. Then, if searcher efficiency trial carcasses that are missed in one search are left in the field for possible discovery on later searches, $p$ and $k$ can estimated simultaneously using EoA. The mathematical description of $k$ is that the searcher efficiency for carcasses on the $i$ th search after arrival is $p_{i}=p k^{i-1}$, and the number of carcasses observed on the $i$ th search after arrival is a binomial random variable $X_{i} \sim \operatorname{binomial}\left(N_{i}, p k^{i-1}\right)$, where $N_{i}$ is the number of carcasses available for discovery on the $i$ th search after arrival. The model is fit via Markov chain Monte Carlo using JAGS (Plummer, 2003) and the $\mathrm{R}$ package $r j$ ags (Plummer, 2016). $k$ is restricted to the interval $[0,1]$. A Jeffreys prior for is used for $p$ (that is, prior $p \sim \operatorname{beta}(0.5,0.5)$ ) and a uniform prior is used for $k$. A burn-in period of 1,000 iterations is used. The modeled joint posterior distribution of $p$ and $k$ is sampled as a component of the simulation of $\hat{g}$ with $n s i m$ iterations, where $n \operatorname{sim}$ depends on the degree of uncertainty in the estimate (appendix A). 


\section{Appendix E. Multiple Classes}

Most fatality estimators use the classical Horvitz-Thompson (H-T) approach (Horvitz and Thompson, 1952) to combine search data from multiple search classes into estimates of the total. In particular, suppose there are $n$ search classes, with carcass counts $X_{1}, \ldots, X_{n}$ and estimated detection probabilities $\hat{g}_{1}, \ldots, \hat{g}_{n}$. Then, the H-T estimator of total mortality would be $\widehat{M}_{H T}=\sum X_{i} / \hat{g}_{i}$, which estimates the total as the sum of estimates for each class. If the $\hat{g}_{i}$ 's and $\sum X_{i}$ are not small and the $\hat{g}_{i}$ 's are unbiased, $\widehat{M}_{H T}$ is approximately unbiased and tends to have a relatively small variance in relation to the expected value. However, if $\sum X_{i}$ is small, the variance of $\widehat{M}_{H T}$ can be quite large in relation to the mean, and the problem is compounded when $\hat{g}_{i}$ 's are small.

As an alternative, EoA pools the counts as $X=\sum X_{i}$ and the detection probabilities as the weighted average of detection probabilities among the classes, $\hat{g}=\sum a_{i} \hat{g}_{i}$, where $a_{i}$ is the assumed fraction of carcasses landing in class $i$. The estimate of total mortality across all classes is then $\widehat{M}_{E o A}=$ $\sum X_{i} / \sum a_{i} \hat{g}_{i}$. Typically, the weights $\left(a_{i}\right)$ are not known, and there is insufficient (or no) data to estimate them. Instead, they are inferred from external information, such as density-weighted proportions estimated for surrogate species (Huso and Dalthorp, 2014); expected effectiveness of fatality reduction measures such as curtailment (Arnett and others, 2011; Marten Law, 2015; Behr and others, 2016; Bulling and Köppel, 2016) or deterrents (Arnett and others, 2013; Sinclair and DeGeorge, 2016); or assumed differences in fatality rates at different sites based on species activity, risk, or fatality rates of surrogate species.

The EoA estimator of overall detection probability across classes is $\hat{g}=\sum a_{i} \hat{g}_{i}$, which has mean $g=E(\hat{g})=\sum a_{i} E\left(\hat{g}_{i}\right)$ and variance $\sigma^{2}=V(\hat{g})=\sum a_{i}^{2} V\left(\hat{g}_{i}\right)$. The distribution of $\hat{g}$ is not known precisely, but it is assumed that $\hat{g} \sim \operatorname{beta}(B a, B b)$, where $B a=\frac{g^{2}}{\sigma^{2}}(1-g)-g$ and $B b=B a(1 / g-1)$, which are the unique parameter values for the beta distribution with mean of $g$ and variance $\sigma^{2}$. 
EoA's pooled estimator, $\widehat{M}_{E o A}$, has substantially smaller variance than that of $\widehat{M}_{H T}$, but uncertainty about the weights, $a_{i}$, introduces potential bias. The two estimators can be compared using the root mean square error (rMSE) as a measure of how close an estimator is to the target on average, where $r M S E=\sqrt{\left(\text { bias }^{2}+\text { variance }\right)}$. The pooling method of combining classes $\left(\widehat{M}_{E o A}\right)$ has the greatest potential for bias when differences among detection probabilities among classes are most pronounced, so we compare the estimators in an especially challenging scenario for EoA: two search classes, $E$ and $H$, with detection probabilities $g_{E}=0.9$ and $g_{H}=0.1$, and associated weights $a_{E}$ and $a_{H}$ with $a_{E}+a_{H}=1$.

The degree of bias in $\widehat{M}_{E O A}$ is a function of how badly the relative weights between the classes are misspecified, and the relative variance is a function of the total number of fatalities, $M$. The rMSE's for the two estimators were compared for a wide range of misspecifications of $a_{E}$ for $M=$ $1,10,100$, and 1000 . When $M$ was small, $\widehat{M}_{E o A}$ outperformed $\widehat{M}_{H T}$ unless $a_{E}$ was badly misspecified (fig. E1 a, b). When $M$ was large, $\widehat{M}_{H T}$ outperformed $\widehat{M}_{E o A}$ unless the assumed $a_{E}$ was close to the actual (fig. E1 c, d). 

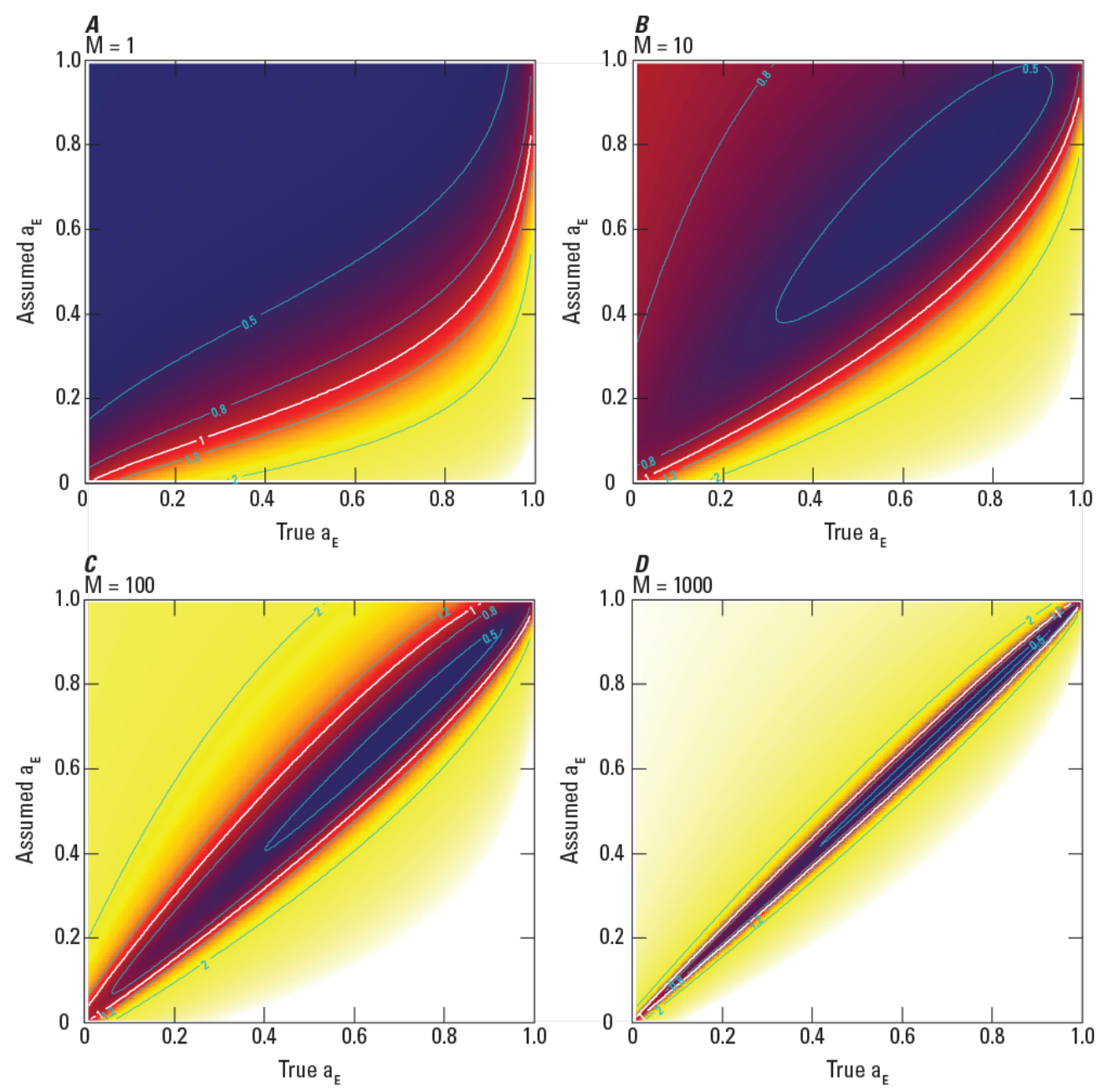

Figure E1. Comparison of EoA's pooling method of combining classes with the classical Horvitz-Thompson approach. Fatality estimation is made for a site with two search classes, $E$ and $H$, with overall detection probabilities of $g_{E}=0.9$ and $g_{H}=0.1$ with relative arrival rates between classes of $a_{E}$ and $1-a_{E}$. Each graph represents the relative root mean square error $\left[\operatorname{rMSE}\left(\widehat{M}_{E O A}\right) / r M S E\left(\widehat{M}_{H T}\right)\right]$ as a function of the true and assumed class weights, $a_{E}$. Heavy white contour line is drawn at 1.0, indicating equal rMSE values for the two estimators. Other contours are drawn at $0.5,0.8,1.2$, and 2.0. Blue indicates regions where $\operatorname{rMSE}\left(\widehat{M}_{E O A}\right)<$ $\operatorname{rMSE}\left(\widehat{M}_{H T}\right)$, favoring $\widehat{M}_{E O A}$, and yellow indicates regions where $r M S E\left(\widehat{M}_{E O A}\right)>\operatorname{rMSE}\left(\widehat{M}_{H T}\right)$, favoring $\widehat{M}_{H T}$. 


\section{Appendix F. Multiple Years}

\section{F.1 Estimation of past years}

The estimator of total mortality over $n$ years, $\widehat{M}$, is identical to the estimator for total mortality across multiple classes: $\widehat{M}=\sum X_{i} / \sum \rho_{i} \hat{g}_{i}$, where $i$ is an index for the year, $X_{i}$ is the number of carcasses observed, $\hat{g}_{i}$ is the estimated detection probability, and $\rho_{i}$ is the (assumed) relative mortality rate.

The estimator of the year-specific mortality rate, $\lambda_{i}$, for year $i$ (in the past) is identical to the estimator for $\lambda$ in the single class module (appendix B). In particular, $\lambda_{i}$ for years $i=1, \ldots, n$ are estimated independently from each year's search data, $\left(X_{i}, \hat{g}_{i}\right)$. The posterior distribution of the annual fatality rate in year $i$ is $P\left(\lambda_{i} \mid X_{i}, \hat{g}_{i}\right)=P\left(X_{i} \mid \lambda_{i}, \hat{g}_{i}\right) P\left(\lambda_{i}\right) / \int P\left(X_{i} \mid \lambda_{i}, \hat{g}_{i}\right) P\left(\lambda_{i}\right) d \lambda_{i}$, where $X_{i} \mid \lambda_{i}, \hat{g}_{i} \sim \operatorname{binomial}\left(M_{i}, \hat{g}_{i}\right), M_{i} \sim \operatorname{Poisson}\left(\lambda_{i}\right)$, and $\hat{g}_{i} \sim \operatorname{beta}\left(B a_{i}, B b_{i}\right)$; and $P\left(\lambda_{i}\right) \propto 1 / \sqrt{\lambda_{i}}$ is the Jeffreys prior for $\lambda_{i}$.

\section{F.2 Projection of future years}

Projection of future mortality begins with estimation of the baseline annual fatality rate, $\lambda$, which would occur in years with $\rho=1$. The posterior distribution of the combined fatality rate through then years of monitoring is $\lambda^{\prime} \mid(X, \hat{g})$. The distribution is calculated for $X=$ the total number of carcasses observed in $n$ years of monitoring and $\hat{g}$ is the estimated overall detection probability for carcass arrivals in years 1 through $n: P\left(\lambda^{\prime} \mid X, \hat{g}\right)=P\left(X \mid \lambda^{\prime}, \hat{g}\right) P\left(\lambda^{\prime}\right) / \int P\left(X \mid \lambda^{\prime}, \hat{g}\right) P(\lambda) d \lambda^{\prime}$, where $X \mid\left(\lambda^{\prime}, \hat{g}\right) \sim \operatorname{binomial}(M, \hat{g}), M \sim \operatorname{Poisson}\left(\lambda^{\prime}\right), \hat{g} \sim \operatorname{beta}(B a, B b)$ (appendix E), and $P\left(\lambda^{\prime}\right) \propto 1 / \sqrt{\lambda^{\prime}}$ is the Jeffreys prior for the Poisson rate parameter, $\lambda^{\prime}$. The estimated baseline fatality rate, $\lambda$, is then defined to be the posterior distribution of $\lambda^{\prime} \mid(X, \hat{g})$, rescaled so that $\lambda$ is the posterior distribution of the fatality rate when $\rho=1$. That is, $P\left(\lambda \leq \lambda^{*}\right)=P\left[\lambda^{\prime} \mid(X, \hat{g}) \leq \sum \rho_{i} \lambda^{*}\right]$. Simulation is used to project the total cumulative number of fatalities through year $n+m$, where $n$ is the number of past years of search data and $m$ is the number of years of fatality projections. First, the cumulative total, $M_{n}$, of past fatalities through year $n$ are drawn from the posterior distribution of $M \mid(X, \hat{g})$. Next, a baseline fatality rate is drawn from the posterior of $\lambda \mid(X, \hat{g})$. Finally, projected annual fatalities, $\widetilde{M}_{j}$, for the next $m$ years $j=1, \ldots, m$ are generated as random draws from a Poisson $\left(\rho_{j} \lambda\right)$ distribution. The total projected fatality through each year $n+j$ for $j=1, \ldots, m$ is then $M_{n, j}=M_{n}+\sum_{y=1}^{j} \widetilde{M}_{y}$. 
The projected estimates of cumulative mortality through year $n+j \in[1, \ldots, m]$ are $\widetilde{M}^{*}$ values derived from the total observed carcass count through year $n$ added to the simulated projected carcass counts, $X_{j} \sim \operatorname{betabinomial}\left(\widetilde{M}_{j}, B a_{j}, B b_{j}\right)$, for $j=n+1, \ldots, n+y$, where $\widetilde{M}_{j}$ is the projected fatality in year $j$, and $B a_{j}$ and $B b_{j}$ are the parameters for the beta distribution characterizing $\hat{g}_{j}$ (as provided by the user). The cumulative total projected mortality in year $n+j, \widetilde{M}_{n, j}^{*}$ is defined as the minimum number of fatalities such that $P\left(\widetilde{M} \leq \widetilde{M}_{n, j}^{*} \mid X=\sum_{i=1}^{n} X_{i}+\sum_{y=1}^{j} X_{y} ; \hat{g}\right) \leq 1-\alpha$ for the user's choice of credibility level, $1-\alpha$.

\section{F.3 Tests on assumed weights, $\rho$}

There are 3 separate tests on the assumed weights used in the Multiple Years module, including a qualitative comparison of the assumed weights versus the fitted weights, a likelihood ratio test on the plausibility of the assumed weights, and a quick test of the potential degree of bias introduced by possible misspecification of the weights. The tests are also applicable to Multiple Class estimates after substituting "class" for "year" and "classwise" for "annual" in the discussion in G.3.1 and G.3.2.

\section{F.3.1 Qualitative comparison of assumed weights and fitted weights}

The assumed weights for each year (as entered by the user) are listed alongside $95 \%$ CIs for fitted weights. The fitted weights are determined by simulation with $n \operatorname{sim}=1,000$ random draws from each posterior distribution of fatality rates, $\lambda_{i} \mid\left(X_{i}, B a_{i}, B b_{i}\right)$ (appendix B), for years $i=1, \ldots, n$ to construct an $n \operatorname{sim} \times n$ array of simulated fatality rates, $\boldsymbol{\Lambda}$. The simulated fatality rates are normalized to give relative weights and scaled to match the scale of the $\rho_{i}$ 's entered by the user. Specifically, for each

simulated year, the fitted $\rho$ 's are given as $\hat{\rho}_{i}=\frac{\lambda_{i}}{\sum \lambda_{i}} \sum \rho_{i}$, where $\rho_{i}$ is the assumed weight for year $i$. The 95\% CI for fitted weight for year $i$ is defined as the 0.025 and 0.975 quantiles of the simulated fitted weight $\hat{\rho}_{i}$. 


\section{F.3.2 Test of plausibility of assumed weights}

The null model assumes that the weights are specified correctly and that the fatality rate is $\lambda_{0} \rho_{i}$ for years $i=1, \ldots, n$. $\lambda_{0}$ is estimated by maximum likelihood. More precisely, $\hat{\lambda}_{0}$ is the value that maximizes $L\left(\hat{\lambda}_{0}\right)=\prod_{i} P\left(x_{i} \mid \hat{\lambda}_{0}, B a_{i}, B b_{i}\right)$, where $X_{i} \mid\left(\hat{\lambda}_{0}, B a_{i}, B b_{i}\right) \sim \operatorname{betabinomial}\left(M_{i}, B a_{i}, B b_{i}\right)$ and $M_{i} \sim$ Poisson $\left(\hat{\lambda}_{0} \rho_{i}\right)$. The alternative model calculates the maximum likelihood estimate $\hat{\lambda}_{i}$ of fatality rate for each year $i=1, \ldots, n$ as the $\hat{\lambda}_{i}$ that maximizes $L\left(\hat{\lambda}_{i}\right)=P\left(x_{i} \mid \hat{\lambda}_{i}, B a_{i}, B b_{i}\right)$, where $X_{i} \sim \operatorname{betabinomial}\left(M_{i}, B a_{i}, B b_{i}\right)$ and $M_{i} \sim \operatorname{Poisson}\left(\hat{\lambda}_{i}\right)$. The test statistic $D=2 \cdot\left[\log \left(\Pi_{i} L\left(\hat{\lambda}_{i}\right)\right)-\right.$ $\left.\log \left(L\left(\hat{\lambda}_{0}\right)\right)\right]$ is assumed to be distributed as $D \sim \chi^{2}(n-1)$ and the $p$-value is given as $P\left(\mathrm{X}_{n-1}^{2}>D\right)$. A small $p$-value is indicative of misspecification of $\rho_{i}$ 's, but that misspecification may or may not introduce bias into the estimates of total fatality $M$ or cumulative fatality rate $\lambda$ (section G.3.3).

\section{F.3.3 Quick test of relative bias}

In many cases, estimates of $\lambda$ and $M$ are extremely robust to assumptions about the $\rho_{i}$ 's. In particular, if the detection probabilities vary little from year to year and the fatality rates are low, the estimates of $\lambda$ and $M$ will be largely unaffected by choices about $\rho_{i}$. A quick test of the potential for bias due to misspecification of $\rho_{i}$ 's is given. The test compares the $1-\alpha$ quantile of the posterior distribution of $\lambda$ under the assumption that the $\rho_{i}$ 's are correctly specified $\left(\lambda_{\rho}^{*}\right)$ with the $1-\alpha$ quantile

of the posterior distribution of $\lambda$ assuming the fitted $\rho_{i}$ 's are correct $\left(\lambda_{\widehat{\rho}}^{*}\right)$ and shows $\lambda_{\rho}^{*} / \lambda_{\widehat{\rho}}^{*}$ in the results table. 


\section{Appendix G. Triggers-Technical Details}

\section{G.1 Long-term trigger}

The long-term trigger fires in year $Y$ if the estimated cumulative mortality $\left(M^{*}\right.$ based on the user-specified $\alpha$ ) through year $y$ exceeds the long-term take limit (T). The calculation of $M^{*}$ (appendix B) is based on the pooled carcass counts $\left(X=\sum_{i=1}^{Y} X_{i}\right.$, where $X_{i}$ is the carcass count in year $\left.i\right)$ and the estimated pooled overall detection probability for carcasses that arrive some time in years 1 through $Y$ (appendix F, section F.1).

\section{G.2 Short-term trigger}

The short-term trigger defined for significance level $\alpha$, threshold $\tau$, and a term of $w$ years fires in year $Y$ if the estimated annual fatality rate $(\lambda)$ over the term exceeds $\tau$ with probability $\geq(1-\alpha)$ according to the posterior distribution of $\lambda$. The posterior is derived from the pooled carcass count $X$ over the term (that is, $X=\sum_{i=Y-w+1}^{Y} X_{i}$, where $X_{i}$ is the carcass count in year $i$, assuming $X_{i}=0$ if $i \leq 0)$ and the estimated pooled detection probability over the term as characterized by $B a$ and $B a$ parameters (section G.1). Specifically:

$$
P(\lambda \mid X, B a, B b)=\frac{P(X \mid \lambda, B a, B b) P(\lambda)}{\int P(X \mid \lambda, B a, B b) P(\lambda) d \lambda}
$$

where $X \sim \operatorname{binomial}(M, \hat{g}), M \sim \operatorname{Poisson}(\lambda w), \hat{g} \sim \operatorname{beta}(B a, B b)$, and $P(\lambda) \propto 1 / \sqrt{\lambda}$ is the Jeffreys prior for Poisson rate. The trigger fires when $P(\lambda>\tau \mid X, B a, B b)>1-\alpha$, which gives strong evidence that the actual fatality rate $\lambda$ exceeded the threshold $\tau$ when a small value of $\alpha$ is used. 


\section{G.3 Reversion trigger}

The reversion trigger defined for significance level $\alpha$, threshold $\tau$, and constraint effect $\rho$ fires in year $Y$ if the estimated annual fatality rate $(\lambda)$ over years 1 through $Y$ is less than $\tau \rho$ with probability $\geq(1-\alpha)$ according to the posterior distribution of $\lambda$. To conduct the test, define the required significance level $\alpha$ and calculate a posterior distribution of the actual take rate $\lambda$ using the (noninformative) Jeffreys prior. Let $X$ be the cumulative total carcass count for year 1 through year $Y$. The posterior of $\lambda$ is conditioned on the pooled carcass count and the estimated detection probability as characterized by $B a$ and $B b$ parameters (section F.1). Specifically:

$$
P(\lambda \mid X, B a, B b)=\frac{P(X \mid \lambda, B a, B b) P(\lambda)}{\int P(X \mid \lambda, B a, B b) P(\lambda) d \lambda}
$$

where $X \sim \operatorname{binomial}(M, \hat{g}), M \sim \operatorname{Poisson}(\lambda Y), \hat{g} \sim \operatorname{beta}(B a, B b)$, and $P(\lambda) \propto 1 / \sqrt{\lambda}$ is the Jeffreys prior for Poisson rate. The trigger fires when $P(\lambda<\tau \rho \mid X, B a, B b)>1-\alpha$, which gives strong evidence that the actual fatality rate $\lambda$ was less than $\tau \rho$ when a small $\alpha$ is used. 


\section{Appendix H. Parameterizations of Distributions Used in the Software}

Weibull Distribution.-The parameterization used for the Weibull $(\alpha, \beta)$ distribution in the Evidence of Absence software is identical to that used in the base package in $\mathrm{R}$ with $\alpha=$ shape and $\beta=$ scale. The probability density function (pdf) and cumulative distribution function (cdf) are given by $f(x)=\frac{\alpha}{\beta} \cdot\left(\frac{x}{\beta}\right)^{\alpha-1} e^{-\left(\frac{x}{\beta}\right)^{\alpha}}$ and $F(x)=1-e^{-\left(\frac{x}{\beta}\right)^{\alpha}}$, respectively, while the mean and variance are $\mu=\mathrm{E} X=\beta \Gamma\left(1+\frac{1}{\alpha}\right)$ and $\sigma^{2}=\mathrm{V} X=\beta^{2}\left[\Gamma(1+2 / \beta)-\Gamma^{2}(1+1 / \alpha)\right]$.

Lognormal Distribution.-The parameterization used for the $\operatorname{Lognormal}(\alpha, \beta)$ is identical to that used in the base package in $\mathrm{R}$ after substituting meanlog $=\beta$ and $\mathrm{sdlog}=\sqrt{\alpha}$. The pdf is given by $f(x)=\frac{e^{-(\log x-\beta)^{2} /(2 \alpha)}}{x \sqrt{2 \pi \alpha}}$, and the mean and variance are $\mathrm{E} X=e^{\beta+\alpha / 2}=\mu$ and $\mathrm{V} X=\left(e^{\alpha}-1\right) e^{2 \beta+\alpha}=\sigma^{2}$.

Log-Logistic Distribution.-The parameterization used for the $\log \operatorname{logistic}(\alpha, \beta)$ is identical to that used by the R statistical package actuar after substituting shape $=\alpha$ and scale $=\beta$. The pdf is $f(x)=\frac{(\alpha / \beta)(x / \beta)^{\alpha-1}}{\left[1+(x / \beta)^{\alpha}\right]^{2}}$, the cdf is $F(x)=\frac{x^{\alpha}}{\beta^{\alpha}+x^{\alpha}}$, and the mean and variance are given by $\mathrm{E} X=\frac{\beta \pi / \alpha}{\sin (\pi / \alpha)}\left(\right.$ if $\alpha>1$; otherwise, undefined) and $\mathrm{V} X=\beta^{2}\left[\frac{2 \pi / \alpha}{\sin (2 \pi / \alpha)}-\left(\frac{\pi / \alpha}{\sin (\pi / \alpha)}\right)^{2}\right]$ (if $\alpha>2$; otherwise, undefined).

Beta Distribution.-The parameterization used for the beta $(\alpha, \beta)$ is identical to that used in the base package in $\mathrm{R}$, with shape1 $=\alpha$ and shape2 $=\beta$. The probability density function is given by $f(x)=\frac{\Gamma(\alpha+\beta)}{\Gamma(\alpha) \Gamma(\beta)} x^{\alpha-1}(1-x)^{\beta-1}$ for $0 \leq \mathrm{x} \leq 1$ and 0 otherwise. The mean and variance are given as $\mu=\frac{\alpha}{\alpha+\beta}$ and $\sigma^{2}=\frac{\alpha \beta}{(\alpha+\beta)^{2}(\alpha+\beta+1)}$, respectively. 


\section{Appendix I. Functions and Data Available from R Command Line}

For the convenience of advanced users, several useful functions in the EoA software package are readily available directly from the $\mathrm{R}$ command line. Some of these functions provide options that are not available in the GUI. For example, user may specify prior distributions for $M$ and $\lambda$ in the calculation of posteriors of $M|(X, g), M|(X, B a, B b), \lambda \mid(X, g)$, and $\lambda \mid(X, B a, B b)$ and may specify a custom arrival function in the calculation of $g$ when searcher efficiency and carcass persistence distribution are known.

\section{I.1 Searcher efficiency model}

\section{Description}

Fitting of the searcher efficiency model (appendix D) using rjags implementation of Markov chain Monte Carlo for given searcher efficiency trial data.

\section{Usage}

pkfit (pkdat, burn $=2000$, n.iter $=2000)$

Arguments

pkdat list containing $\mathrm{n}=$ number of searches in field trials, $\mathrm{M}=$ number of carcasses available for discovery in each search (vector of length $n$ ), and $x=$ number of carcasses discovered in each search (vector of length $n$ )

burn number of burn-in iterations to establish the stationary distribution of $(p, k)$

n.iter number of total iterations in the Gibbs sample

Details

User is given flexibility to explore the fitting of the model of searcher efficiency as a function of the number of times a carcass has been missed in previous searches. Further fine tuning of parameters for fitting the model can be attained by invoking JAGS functions directly using EoA's model for $(p, k)$, which is stored in eoa: : pkmod.

Value

The function returns an $n$. iter $\times 2$ array of simulated $(p, k)$ representing the joint distribution of the estimated searcher efficiency parameters. The array is a subset of a mcmc object returned from rjags: : coda. samples and contains a brief description of the data. 


\section{I.2 Carcass persistence model}

\section{Description}

Functions to fit persistence models to data from carcass persistence field trials (section 2.1.3), to simulate persistence parameters and/or persistence times from fitted persistence models (appendix C), and to calculate probability of carcass persistence from arrival at a random time during one interval until a search at a specified later time.

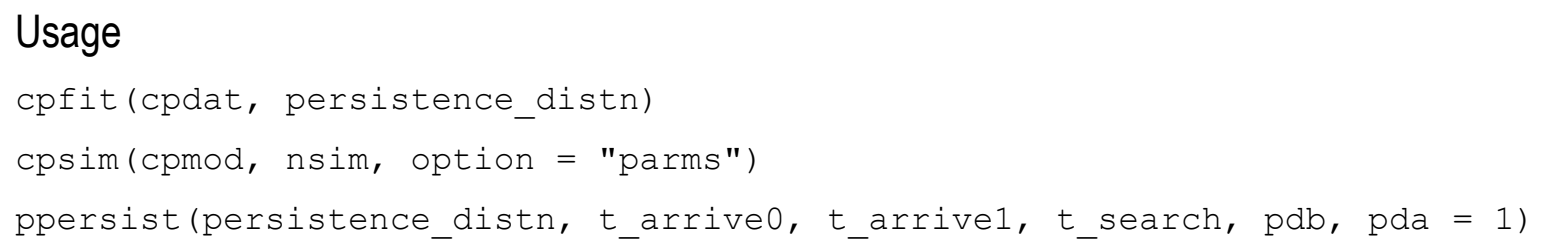
last time the carcass was observed, and second column gives the first time the carcass was noted missing. If right censored, enter Inf in the second column.

persistence_distn name of the persistence distribution to be fit

cpmod a fitted persistence model

nsim desired number of random draws from the persistence distribution

option indicator to simulate parameter values ("parms") or persistence times ("times")

t_arrive0 beginning of time interval for carcass arrival

t_arrive1 end of time interval for carcass arrival

$t$ _search time that search is conducted ( $t$ _arrive1 $\leqslant t_{-}$search)

pda, pdb shape and scale parameters for persistence distribution (appendix $\mathrm{H}$ )

Details

Persistence models are fit with cpfit using the tools provided in the survival package (Therneau, 2015). persistence_distn must be specified as "Exponential", "Weibull", "Log-Logistic", or "Lognormal".

The cpsim function with option = "parms" can be used to account for uncertainty in the estimates of persistence distribution by simulating ns im random draws from the fitted model, cpmod, which is an object of class survreg (as returned by the cpfit function in EoA or the survreg function in the survival package (Therneau, 2015)).

Similarly, cpsim function with option = "times" can be used to generate random carcass persistence times after accounting for uncertainty in the persistence distribution parameters and for uncertainty in persistence times for given parameter values. 
The probability of a carcass persisting until a search at time $t$ _search after arriving at a random time in the interval [t_arrive0, t_arrive1] and assuming persistence times follow a persistence_distn persistence distribution with shape and scale parameters $=$ pda and pdb, respectively, or simply pdb $=$ mean persistence time if persistence_distn = "Exponential". The calculation is performed as $r_{i j}$ as discussed in appendix A, where $i$ is the arrival interval and $j$ is the search time and arrival distribution is assumed uniform within the arrival interval. The function is vectorized for both the times and persistence parameters and assumes that $t$ arrive0, $t$ arrive1, and $t$ search are vectors of length $N_{t} \geq 1$ and pda and pdb are vectors of length $N_{p} \geq 1$.

Value

cpfit returns a fitted persistence model of class survreg (Therneau, 2015), which can be used as the cpmod argument in function cpsim.

cpsim returns an nsim $\times 2$ array of simulated persistence shape and scale parameters or an nsim-vector of simulated persistence times, depending on whether option = "parms" or "times".

ppersist returns an $N_{t} \times N_{p}$ array of probabilities of a carcass persisting from its arrival at a random time in intervals [t_arrive0, t_arrive1] until a search at time $t_{-}$search, where $N_{t}$ is the length of the

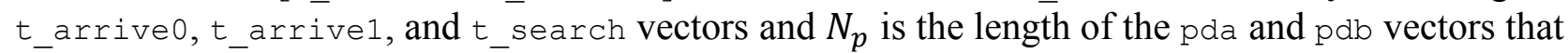
define the assumed persistence distribution(s).

\section{I.3 Arrival Function Builder}

\section{Description}

Tools for constructing a carcass arrival distribution (appendix A) comprised of three components, including a uniform distribution and two beta distributions. The compound arrival distribution is highly flexible and can accommodate a wide variety of patterns of seasonal variation in mortality rates. For example, a constant, small underlying risk of mortality throughout the year with peaks in risk associated with spring and fall migration could be modeled as: (1) a uniform distribution to represent the persistent underlying risk, plus (2) beta distributions to represent the increased risk in spring and fall. Arrival functions that are produced using the arrival function builder can be used in the $\mathrm{R}$ command line function to calculate the overall detection probability, $g$ (section I.4). 


\section{Usage}

The data and functions used in the arrival function builder are organized in an R6 class called arrivalmodel. An arrivalmodel object is created in $\mathrm{R}$ by invoking the new method for the class:

arrmod<-arrivalModel\$new (parms = NULL, duration = NULL)

Existing arrivalModel objects can be edited via the buildModel method:

arrmod\$buildModel ()

\section{Arguments}

parms initial arrival function parameters. If parms is missing or NULL, arrival function builder window opens with a generic arrival function, which provides a convenient starting point for building a custom arrival function. The generic arrival function is arbitrary and should in no way be considered typical or recommended. Alternatively, initial arrival parameters may be explicitly specified in the parms argment. Format is either an existing arrivalmodel object or a list containing parameter values to define a compound arrival function, which include arrcomponents (logical 3-vector indicating whether the model includes uniform, beta1, and beta2 components, respectively); lwr.u and upr.u (lower and upper bounds of the uniform component); lwr.p1, upr.p1, lwr.p2, and upr.p2 (lower and upper bounds of the beta 1 and beta 2 components); a.p1, b.p1, a.p2, and b.p2 ( $\alpha$ and $\beta$ parameters of the beta1 and beta 2 components); and wt.u, wt.p1, and wt.p2 (relative contributions of each component to the compound arrival function).

duration upper bound on the $x$-axis of the of the arrival function builder graph

\section{Details}

When a new arrivalModel is created, the arrival function builder window opens, allowing the user to define the components and parameters (bounds, weights, symmetries, aspects) of a compound arrival function (fig. I1). The components (uniform, beta1, beta2) are selected via the radio buttons in the upper left region of the window, and the parameters governing the shapes of the components (bounds, weights, symmetries, and aspects) are controlled by the sliders. Parameter values are displayed in the table in the upper left corner of the window. Component options, parameter values, and the resulting arrival function are saved to the arrivalmodel object (arrmod in this example) and the window closed when Save is clicked. The newly constructed arrival function can be accessed directly and used in the same ways as other R functions. Some examples are given below.

Example 1: graph the arrival function

$\mathrm{xx}<-0: 365$

plot (xx, arrmod\$arrfun(xx)) \# graph of the arrival function 


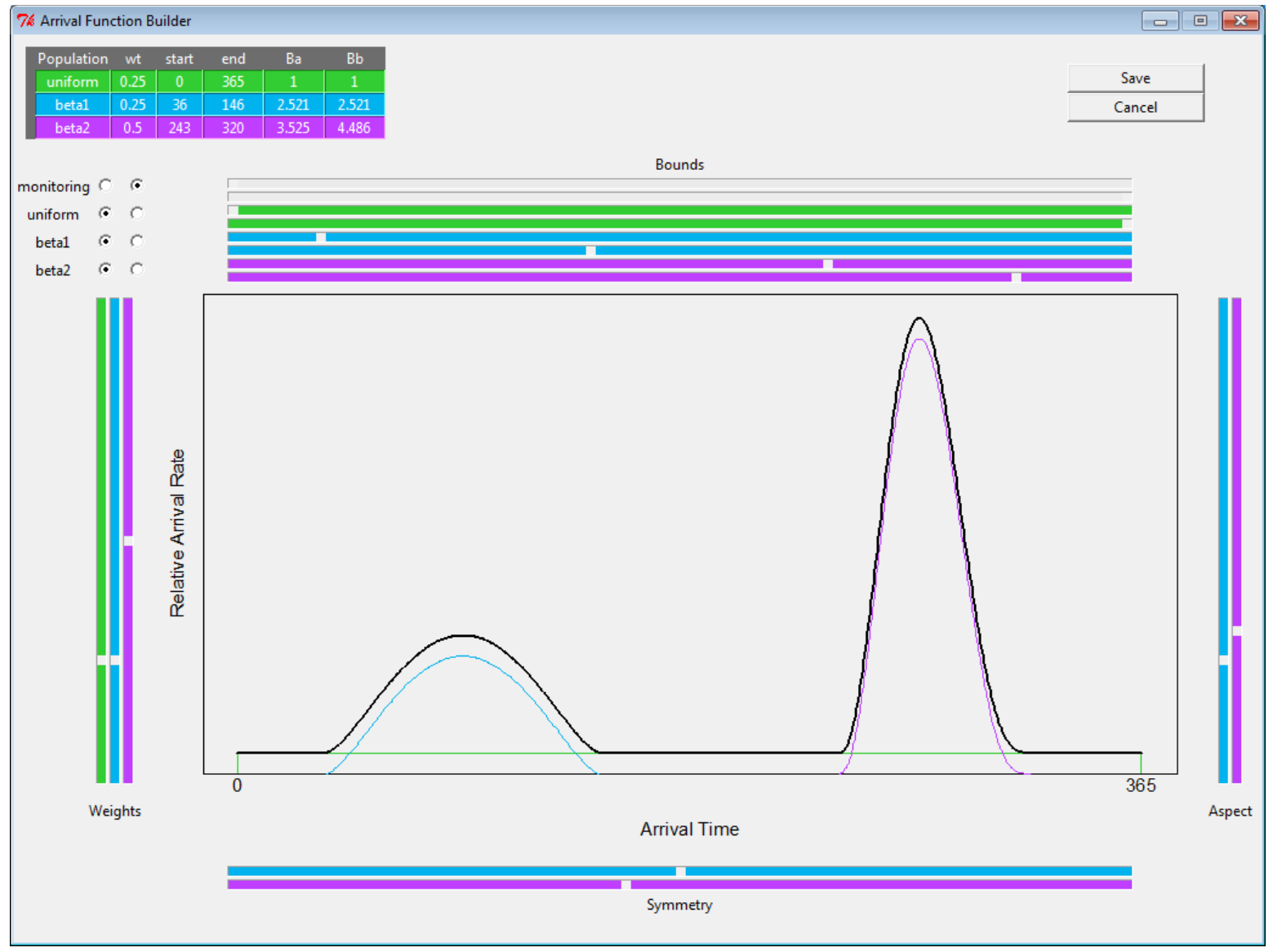

Figure 11. Arrival function builder. The relative arrival rate of carcasses through period of possible times of arrival is modeled as a sum of a uniform component and two beta distribution components. User may select which components to include in the model and adjust characteristics of each component using the sliders located outside the margins of the graph. The compound arrival function is represented by a heavy black curve, and the contributions of each component are represented by colored lines.

Example 2: calculate the fraction of carcasses arriving in the first 150 days

integrate (arrmod\$arrfun, lower=0, upper=150) \$value

In addition, the arrivalmodel object (arrmod in this example) can be used as the arrdat argument in the calcg.fixed (gdat, arrdat $=$ NULL, arrSimplify $=T$ ) function (appendix I.4).

A monitoring period can be superimposed on the arrival graphs (fig. I2) within the arrival function builder by activating the "monitoring" option in the upper left region of the window. Sliders can be used to adjust the bounds of the monitoring period, with the fraction of carcasses that arrive before, during, and after the monitored period displayed near the top of the graph. 


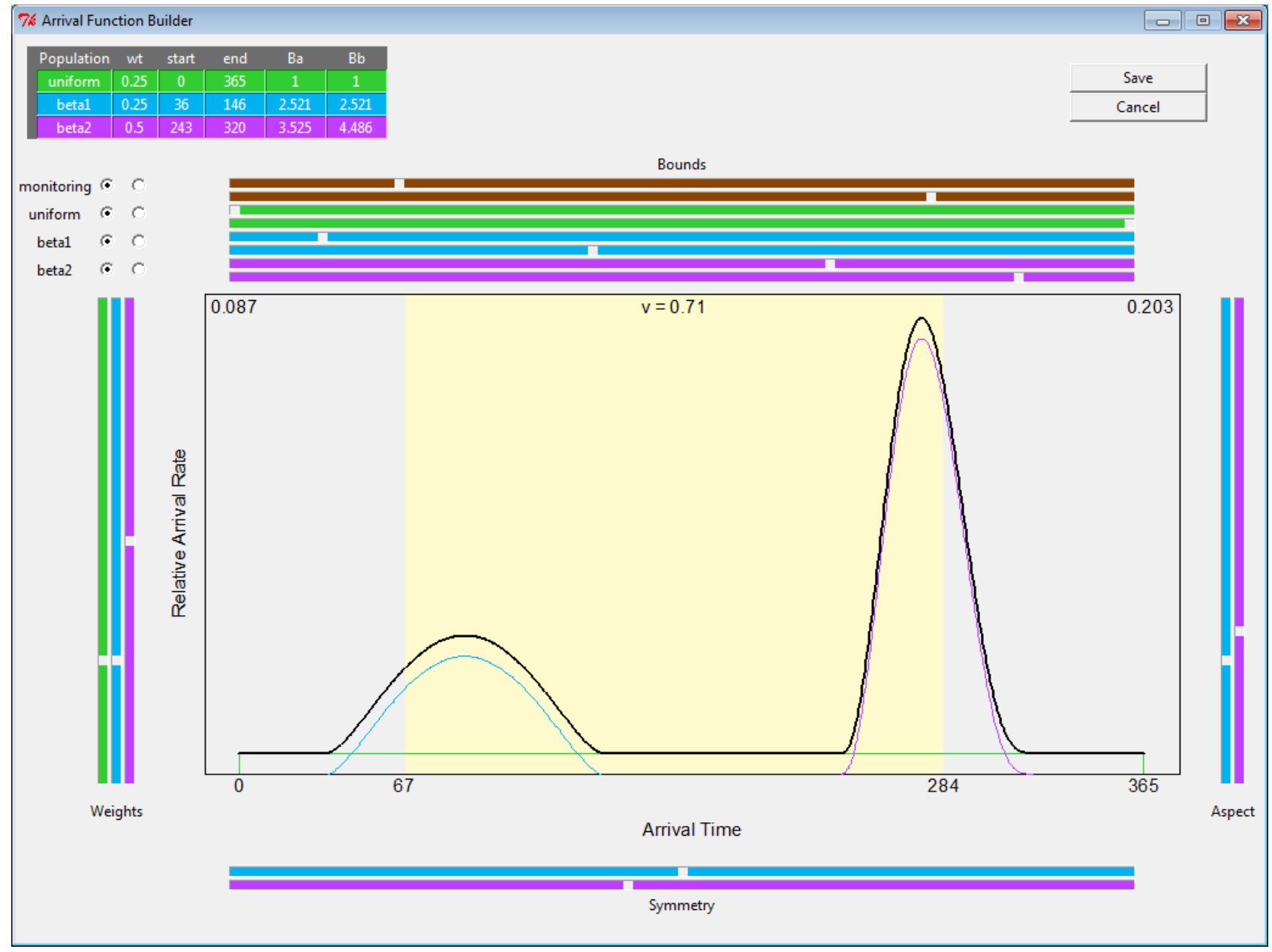

Figure 12. Arrival function builder with the monitoring option selected. The monitoring period is highlighted in the figure. Bounds of the monitoring period can be adjusted using the sliders above the graph. Bounds are given on the $x$-axis below the graph, and the fractions of carcasses arriving in each period are shown near the the top of the graph. The monitoring period depicted in the figure begins 67 days after the beginning of the arrival period and extends through 284 days after arrivals begin. The fraction of carcasses arriving within the monitored period is $\boldsymbol{v}=0.71$, while 0.087 arrive before the first search and 0.203 arrive after the last search. 
Value

Upon closing, the arrival function builder window returns a compound arrival function with the specified parameters wrapped in an R6 object. The function is stored as the arrfun member and component parameters are stored in a list with names matching those of the parms argument. For example, if arrmod is the R6 object, the arrival function arrmodsarrfun ( $t$ ) is the PDF of arrivals as a function of days since the beginning of the arrival period, and the uniform component would extend from arrmod\$lwr.u to arrmod\$upr.u (assuming arrmod\$arrcomponents [1] = TRUE).

\section{I.4 Estimation of $g$}

Description

Calculate the overall detection probability, $g$, for a given, fixed set of arrival, persistence, and search parameters.

Usage

calcg.fixed (gdat, arrdat $=$ NULL)

Arguments

gdat list of carcass persistence and search parameters required to calculate $g$, including:

a $\quad$ spatial coverage (section 2.1.1)

$\mathrm{v} \quad$ temporal coverage (section 2.1.1)

$\mathrm{p}, \mathrm{k} \quad$ searcher efficiency parameters (section 2.1.2)

samtype "Formula" or "Custom"

Isam, nsearch search interval, number of searches (if samtype="Formula")

days vector of search days, starting at 0 (if samtype="Custom")

persistence_distn name of persistence distribution: "Exponential", "Weibull", "Log-Logistic", or "Lognnormal"

pda, pdb $\quad \alpha$ and $\beta$ parameters of persistence distribution (appendix $\mathrm{H}$ ) 
list that defines (optional) arrival function characteristics. With arrdat $=$ NULL, arrivals are assumed to be uniform within the monitored period. Alternatively, arrdat may be a vector of relative arrival rates within each search interval (length (arrdat) = nsearch or length (days) - 1). arrdat may also be an arrivalModel object (appendix I.3) or a list that includes arrfun = either a vector of relative arrival rates within each search interval, "Uniform", or an arrival function. If arrfun is a vector, the fractions of carcasses arriving before and after the monitored period must be provided as arrmiss 0 and arrmissf, respectively. If arrfun is a function (or "Uniform"), the arrdat must include so $=$ start of monitoring period (in number of days after the beginning of arrivals), duration = length of monitoring season, and arrSimplify $=$ TRUE or FALSE. arrSimplify indicates whether or not to make the simplifying assumption that arrivals are uniform within each search interval. The default is to set arrSimplify = TRUE if no value is specified.

\section{Details}

The overall detection probability is calculated (appendix A) for the given parameter set. calcg.fixed (gdat, arrdat $=$ NULL) can accommodate non-uniform arrival functions, such as those included in arrivalModel or custom user-defined arrival functions.

Value

Function returns a list of detection probabilities for "Full site, full year", "Full site, monitored period", and "Searched area, monitored period" (section 2.3.1).

\section{I.5 Calculation of posterior distribution of $M$}

\section{Description}

Calculation of the posterior distribution of total mortality $(M)$ given the carcass count, overall detection probability, and prior distribtion. Calculation of summary statistics from the posterior distribution of $M$.

Usage

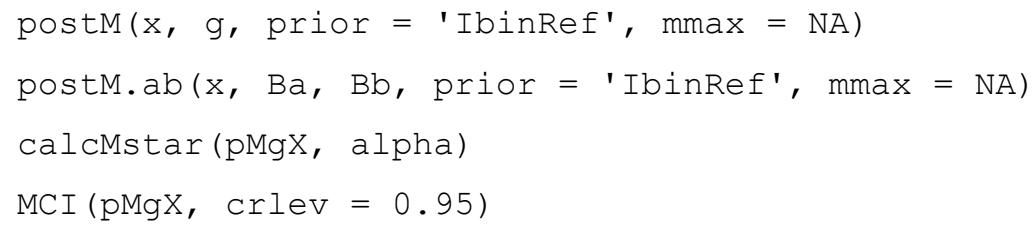


Arguments

$\begin{array}{ll}\mathrm{x} & \text { carcass count } \\ \mathrm{g} & \text { overall carcass detection probability } \\ \mathrm{Ba}, \mathrm{Bb} & \text { parameters for beta distribution characterizing the posterior distribution of } g \\ \text { prior } & \text { prior distribution of } M \\ \text { pMgX } & \text { posterior distribution of } M \\ \text { crlev, alpha } & \text { credibility level }(1-\alpha) \text { and its complement }(\alpha)\end{array}$

Details

The functions postm and postm.ab return the posterior distributions of $M \mid(X, g)$ and $M \mid(X, B a, B b)$, respectively, with options to specify prior distribution for $M$ and limit for truncating the prior to disregard implausibly large values of $M$ and make the calculations tractable. Use postm when $g$ is fixed and known; otherwise, use postm. ab when uncertainty in $g$ is characterized in a beta distribution with parameters $B a$ and $B b$ (section 2.3.1). The non-informative, integrated reference prior for binomial random variables (appendix B) is the default (prior = "IbinRef"). Other options include "binRef", "IbetabinRef", and "betabinRef", which are the non-integrated and integrated forms of the binomial and betabinomial reference priors (Berger and others, 2012). For values of $X$ greater than 2, the integrated and non-integrated reference priors give virtually identical posteriors. However, the nonintegrated priors assign infinite weight to $m=0$ and return a posterior of $P(M=0 \mid X=0, \hat{g})=1$ when $X=0$, implying absolute certainty that the total number of fatalities was 0 if no carcasses were observed. In addition, a uniform prior may be specified by prior = "uniform". Alternatively, a custom prior may be given as a 2-dimensional array with columns for $m$ and $P(M=m)$, respectively. The first column $(m)$ must be sequential integers starting at $m=0$. The second column gives the probabilities associated with $m$, which must be non-negative and sum to 1 .

The named priors ("IbinRef", "binRef", "IbetabinRef", and "betabinRef") are functions of $m$ and defined on $m=0,1,2, \ldots$ without upper bound. However, the posteriors can only be calculated for a finite number of $m$ 's up to a maximum of mmax, which is to set by default to the smallest value of $m$ such that $P(X \leq x \mid m, \hat{g})<0.0001$, where $x$ is the observed carcass count or may be specified by the user.

Value

The functions postm and postm. ab return the posterior distributions of $M \mid(X, g)$ and $M \mid(X, B a, B b)$, respectively. The functions calcMstar and MCI return $M^{*}$ value (section 1.1.2) and credibility interval for the given posterior distribution, pMgX (which may be the return value of postm or postm.ab) and $\alpha$ value or credibility level. 


\section{I.6 Calculation of posterior distribution of $\lambda$}

\section{Description}

Calculation of the posterior distribution of mortality rate $(\lambda)$ given the carcass count, overall detection probability, and prior distribtion.

Usage

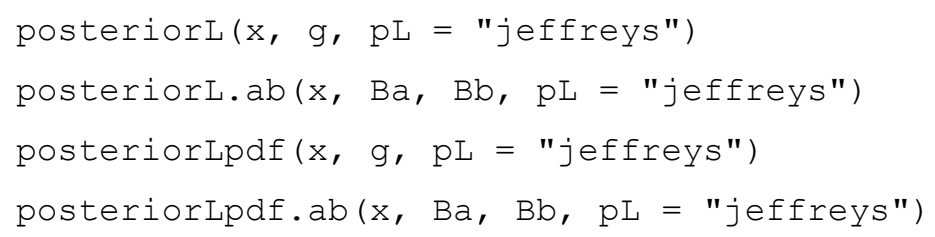

Arguments

$\begin{array}{ll}\mathrm{x} & \text { carcass count } \\ \mathrm{g} & \text { overall carcass detection probability } \\ \mathrm{Ba}, \mathrm{Bb} & \text { parameters for beta distribution characterizing the posterior distribution of } g \\ \mathrm{pL} & \text { prior distribution of } M\end{array}$

Details

The functions posteriorL and posteriorL.ab return the posterior CDFs of $\lambda \mid(X, g)$ and $\lambda \mid(X, B a, B b)$, respectively, and posteriorLpdf and posteriorLpdf ab return the posterior PDFs. User is given the option to specify a prior distribution for $\lambda$, which may be $\mathrm{pL}=$ "jeffreys" for the Jeffreys prior (appendix B), $\mathrm{pL}=$ "uniform" for a uniform prior,or $\mathrm{pL}=$ a custom prior distribution entered as a function of $\mathrm{L}$ (for example $\mathrm{pL}=$ function $(\mathrm{L})$ ). Custom priors must be non-negative and vectorized. 


\section{Value}

The functions return the posterior distribution of $\lambda$ as a vectorized function. For example, to compare graphs of the posterior PDFs derived from Jeffreys prior, uniform prior, and the Jaynes prior $[P(\lambda) \propto$ $1 / \lambda$ ] for detection probability $g=0.20$ and $\mathrm{x}=4$ carcasses, enter the following commands into $\mathrm{R}$ :

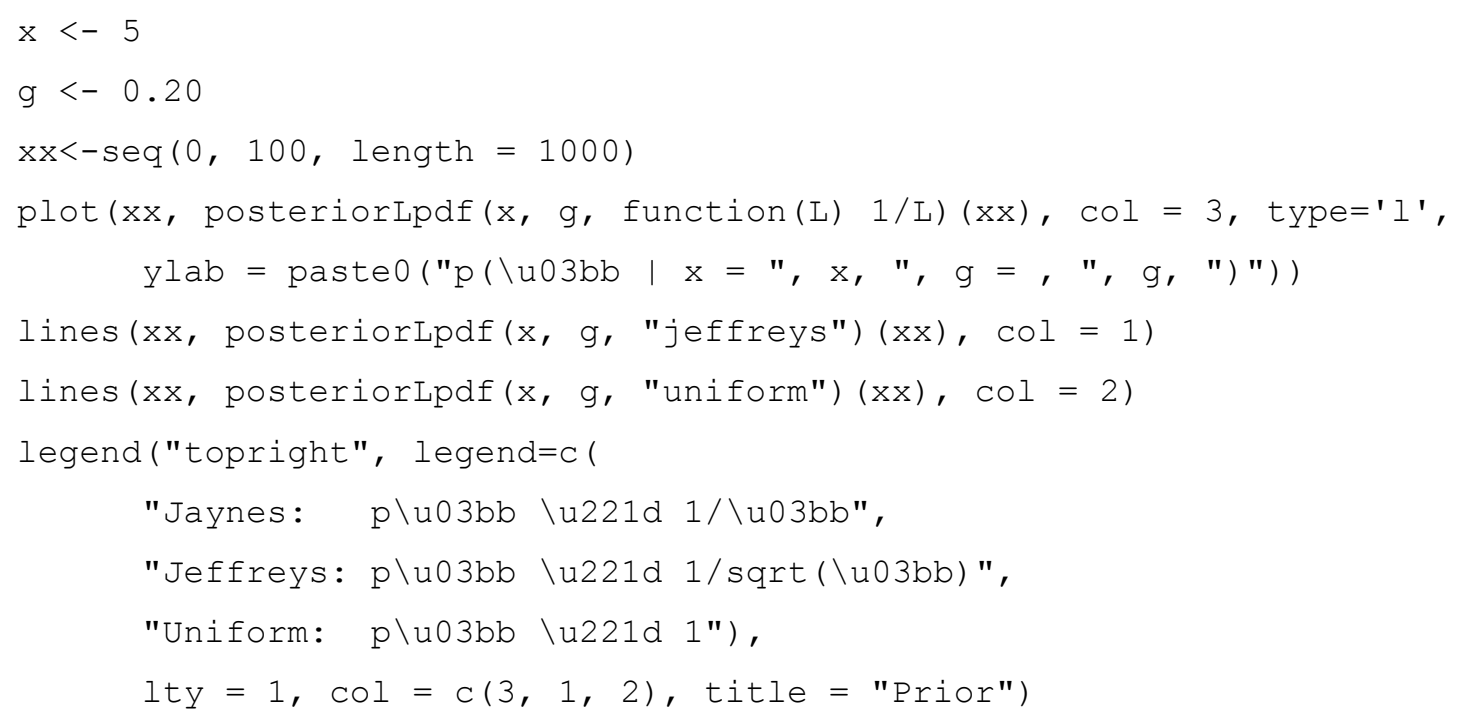

\section{I.7 Default data sets}

A number of generic data sets are provided as templates for inputs and as starting points for exploration and discussion of software features and capabilities. None of the generic data sets should be considered as typical or in any way recommended.

pkdatDefault

CPdataDefault

parmsDefault

gdatDefault searcher efficiency field trial data for pkfit (section I.1)

carcass persistence field trial data for cpfit (section I.2)

compound arrival function parameters for arrivalmodel (section I.3)

required parameters for estimate of $g$ using calcg. fixed (section I.4) 


\section{Appendix J. Conversion Calculator}

To convert from beta distribution $B a$ and $B b$ parameters to $\hat{g}$ with $95 \% \mathrm{CI}$, the conversion calculator sets $\hat{g}=B a /(B a+B b)$, which is the mean of the beta distribution, and gives the 0.025 and 0.975 quantiles of the beta distribution with parameters $B a$ and $B b$ as the bounds of the $95 \%$ CI for $g$. In $\mathrm{R}$, the $95 \% \mathrm{CI}$ is qbeta $(\mathrm{p}=\mathrm{C}(0.025,0.975)$, shape1 $=\mathrm{Ba}$, shape2 $=\mathrm{Bb})$.

Conversion from $\hat{g}$ with $95 \%$ CI to beta distribution with $B a$ and $B b$ parameters is not exact because there is no guarantee that there exists a beta distribution with mean of $\hat{g}$ and the given $95 \%$ CI. The conversion calculator finds the $B a$ and $B b$ parameters for a beta distribution with a mean of $\hat{g}$ and standard deviation equal to $\frac{g_{u p r}-g_{l w r}}{4}$, where $g_{u p r}$ and $g_{l w r}$ are the user-defined bounds on the $95 \%$ CI for $g$. The explicit solution is $B a=16\left(\frac{\hat{g}}{g_{u p r}-g_{l w r}}\right)^{2}(1-\hat{g})-\hat{g}$ and $B b=B a\left(\frac{1-\hat{g}}{\hat{g}}\right)$. 


\section{Appendix K. Comparison with Other Estimators of Detection Probability}

When restricted to a single search class, the EoA estimator of $g$ is generalized in that it allows for non-constant searcher efficiency, scavenging, and arrival rates. It is closely akin to Wolpert's estimator (Wolpert, 2012, 2015; Wolpert and Coleman, 2015) and includes the Shoenfeld (2004), Huso (2011), and Korner-Nievergelt and others (2011) estimators as special cases.

When there is more than one search class, the EoA model estimates $g$ for combined classes as described in appendix E. The other models do not estimate a combined $g$ but rather estimate total mortality $M$ as the sum of the estimated mortalities for each class or site (Horvitz and Thompson, 1952). For small counts, the EoA approach to combining classes tends to have smaller errors than the HorvitzThompson approach, but with larger counts the Horvitz-Thompson approach to combining classes tends to be more reliable.

\section{K.1 Wolpert: more comprehensive model for searcher efficiency, Weibull persistence}

Wolpert (2012) provides explicit formulas to accommodate exponential, Weibull, lognormal, and log-logistic carcass persistence distributions and to accommodate decreases in searcher efficiency both as carcasses age and as they are missed in prior searches. The acmeR (Wolpert and Coleman, 2015) package for $\mathrm{R}$ is a software implementation of the model that assumes uniform arrivals, a Weibull persistence distribution, and a 3-parameter model of the changes in searcher efficiency following carcass arrival. EoA offers a wider variety of options for persistence and employs a simpler model for searcher efficiency. However, when restricted to a single search class, the differences between the models are slight. 


\section{K.2 Shoenfeld: $k=1$, exponential persistence, uniform arrivals, infinite number of equal-length search intervals}

If $k$ is assumed to be 1, persistence distribution is assumed to be exponential, arrivals are assumed to be uniform, and search intervals are of constant length $I$, the estimator (appendix A, eqn. 1) simplifies considerably. To start, define:
$r_{0}=$ probability carcass persists from arrival until first search after arrival
$r=$ probability carcass persists through an entire search interval
$C=$ the event that the carcass is observed
$A_{h}=$ the event that the carcass arrives when there are $h$ searches remaining

To calculate $P(C)=$ the probability of observing a carcass that arrives in the searched area during the monitored period, note that the probability of detection depends on arrival time because carcasses that arrive earlier in the season will be available for detection in more searches than carcasses that arrive later in the season. Thus, we calculate $P(C)$ by conditioning on the number of searches remaining until the end of the monitored period: $P(C)=\sum P\left(C \mid A_{h}\right) P\left(A_{h}\right)$. To calculate each term $P\left(C \mid A_{h}\right)$, we partition by search occasion: $C_{j}=$ the event that the carcass is observed during the $j$ th search after arrival. Then, the probability of observing the carcass on the first search after arrival would be $P\left(C_{1}\right)=r_{0} p$. Because persistence is assumed to be exponential and search intervals are of constant length, the probability of persisting through any full search interval is identically $r$, regardless of how many intervals a carcass has already persisted through. Therefore, the probability of persisting until search $j$ after arrival is $r_{0} r^{j-1}$ because the carcass must persist until the first search after arrival (with probability $r_{0}$ ) and then persist through $j-1$ additional full intervals (with probability $r^{j-1}$ ). To be detected on the $j$ th search, an unscavenged carcass is not detected in any of the first $j-1$ searches (with probability $(1-p)^{j-1}$ ) and is detected on the $j$ th search (with probability $p$ ). Thus, the probability that a carcass is observed during the $j$ th search after arrival is $P\left(C_{j}\right)=r_{0} p[(1-p) r]^{j-1}$. With this, the probability of observing a carcass that arrives with $h$ searches remaining in the monitored period is: $P\left(C \mid A_{h}\right)=\sum_{j=1}^{h} P\left(C_{j} \mid A_{h}\right)=r_{0} p \sum_{j=0}^{h-1}[(1-p) r]^{j}$. This yields $P\left(C \mid A_{h}\right)=r_{0} p \frac{1-[(1-p) r]^{h}}{1-(1-p) r}$ after simplifying the geometric series. 
If, in addition to assumptions that $k=1$, persistence is exponential, search interval length is constant, and arrivals rate is constant, it is further assumed that there are an infinite number of searches, then the detection probability does not depend on how many searches remain in the monitored period and the probability of observing a carcass does not depend on which interval it arrives in, so $g=$ $P(C)=\lim _{i \rightarrow \infty} P\left(C \mid A_{i}\right)=\lim _{i \rightarrow \infty} r_{0} p \frac{1-[(1-p) r]^{i}}{1-(1-p) r}=r_{0} p \frac{1}{1-(1-p) r}$. Now, $r_{0}$ and $r$ can be calculated in terms of the exponential persistence rate parameter, $\lambda$. Substituting $r_{0}=\int_{0}^{I} S(t-I) v(t) d t=$ $\int_{0}^{I} e^{-\lambda(t-I)} d t / I=\frac{1-e^{-I \lambda}}{I \lambda}$ and $r=e^{-I \lambda}$ into the equation and simplifying, we get Shoenfeld's (2004) estimator: $\hat{g}=\frac{p}{I \lambda} \cdot \frac{1-e^{-I \lambda}}{1-e^{-I \lambda}+p e^{-I \lambda}}=\frac{p}{I \lambda} \cdot \frac{e^{-I \lambda}-1}{e^{-I \lambda}-1+p}$.

\section{K.3 Huso: $\boldsymbol{k}=\mathbf{0}$}

The assumption that $k=0$ implies that all carcasses that are observed during search $j$ arrived in interval $j$, which occurs if carcasses that are missed in one search have zero probability of being found in later searches or if found carcasses that arrived before the search prior to the search in which they were found are disregarded and estimation is performed using the fresh carcasses only. If it is further assumed that carcass arrival rate and search interval length $(I)$ are constant, then $\hat{g}=\sum_{j=i}^{n} \sum_{i=1}^{n}\left[\prod_{s=0}^{j-i-1}\left(1-k^{s} p\right) k^{j-i} p\right] \int_{t_{i-1}}^{t_{i}} S\left(t_{j}-t\right) v(t) d t=p\left(1-\frac{\int_{0}^{I} S(t) d t}{I}\right)$, which is the Huso (2011) estimator of $\hat{g}$ (without the correction factor for long search intervals).

In practice, the Huso (2011) estimator is normally used when the number of carcasses observed is not small. A separate $\hat{g}_{i}$ is calculated for each observed carcass, and the total number of carcasses is estimated as $\widehat{M}=\sum_{i=1}^{X} 1 / \hat{g}_{i}$, where $X$ is the total carcass count. In this case, the assumption of constant search interval is not needed and the assumption of uniform arrivals can be relaxed to uniform arrivals within search intervals.

\section{K.4 Korner-Nievergelt and others: exponential persistence and pulsed or uniform arrivals}

The Korner-Nievergelt and others (2011) estimator for $\hat{g}$ is identical to EoA's estimator with exponential persistence and carcasses arriving in pulses at the beginnings of search intervals. However, in the R package carcass (Korner-Nievergelt and others, 2015) the model is updated to be able to accommodate uniform arrivals and the Cox proportional hazard persistence distribution. 

Publishing support provided by the U.S. Geological Survey Science Publishing Network, Tacoma Publishing Service Center

For more information concerning the research in this report, contact the Director, Forest and Rangeland Ecosystem Science Center U.S. Geological Survey

777 NW 9th St., Suite 400

Corvallis, Oregon 97330

https://fresc.usgs.gov 
